\title{
LUNAR ROBOTIC PRECURSOR MISSIONS USING ELECTRIC PROPULSION
}

\author{
By \\ Richard Gerard Winski \\ Thesis submitted to the Faculty of the \\ Virginia Polytechnic Institute and State University in partial \\ fulfillment of the requirements for the degree of \\ Master of Science \\ in \\ Aerospace Engineering \\ Department of Aerospace and Ocean Engineering \\ Blacksburg, Virginia \\ October 2006 \\ Dr. Joseph Wang, Committee Chair \\ Dr. Bernard M. Grossman, Committee Member \\ Dr. Robert Moses, Committee Member
}

(C) Copyright by Richard Gerard Winski, 2006

Keywords: Lunar Mission Analysis, Electric Propulsion, Lunar Robotic Precursor 


\title{
Lunar Robotic Precursor Missions Using Electric Propulsion
}

\author{
Richard G. Winski
}

\begin{abstract}
A trade study is carried out for the design of electric propulsion based lunar robotic precursor missions. The focus is to understand the relationships between payload mass delivered, electric propulsion power, and trip time. The results are compared against a baseline system using chemical propulsion with $\mathrm{LOX} / \mathrm{H}_{2}$. The major differences between the chemical propulsion based and electric propulsion based systems are presented in terms of the payload mass and trip time. It is shown that solar electric propulsion offers significant advantage over chemical propulsion in delivering non-time critical payloads to lunar orbit.
\end{abstract}




\section{Table of Contents}

Table of Contents

List of Tables $\quad$ v

Acknowledgment vi vi vi vis

List of Figures vii

List of Symbols $\quad$ xi

List of Nomenclature $\quad$ xii

1 Introduction and Background 1

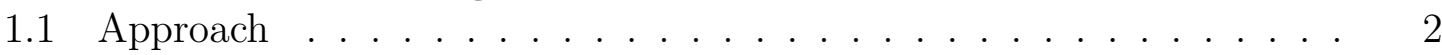

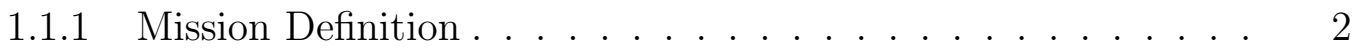

1.1.2 High-Thrust and Low-Thrust Trajectories . . . . . . . . . . 3

2 Mass Models 5

2.1 Robotic Precursor System Mass Model . . . . . . . . . . . . . 5

2.2 Electric Robotic Precursor System Mass Model . . . . . . . . . . . . 6

2.2.1 Spacecraft Model . . . . . . . . . . . . . . . . . 6

2.2.2 Attitude Determination and Control System . . . . . . . . . 6

2.2.3 Guidance Navigation and Control System . . . . . . . . . . . 7

2.2.4 Power System . . . . . . . . . . . . . . . . . . . 7

2.2.5 Propulsion System . . . . . . . . . . . . . . . . . . . . 7

2.2.6 Structural System . . . . . . . . . . . . . . . . 8

2.2.7 Telecommunications System . . . . . . . . . . . . . . 99 9

2.2.8 Thermal Management System . . . . . . . . . . . . . . 9

2.3 Baseline Robotic Precursor System . . . . . . . . . . . . . . . 10

3 Electric Robotic Precursor System 12

3.1 Overview . . . . . . . . . . . . . . . . . . . 12

3.1.1 Diminishing Returns ............... 17 
3.2 Near Term . . . . . . . . . . . . . . . . . . . . . . . . . 22

3.2.1 Diminishing Returns - Near Term . . . . . . . . . . . . 26

3.2 .2 Possible Payloads . . . . . . . . . . . . . . . . . . . 28

3.3 Mid Term . . . . . . . . . . . . . . . . . 33

3.3.1 Diminishing Returns - Mid Term . . . . . . . . . . . 36

3.3 .2 Possible Payloads . . . . . . . . . . . . . . . . . . . 37

3.4 Far Term . . . . . . . . . . . . . . . . . . . . . . 42

3.4.1 Diminishing Returns - Far Term . . . . . . . . . . . . . . 45

3.5 Discussions . . . . . . . . . . . . . . . . . . . . . . . 50

3.5 .1 Sensitivities ..................... 50

3.5.2 Initial Orbit Considerations . . . . . . . . . . . . . . . . 52

3.5.3 Technology Level Considerations . . . . . . . . . . . . 53

3.5 .4 Overall Program Goals . . . . . . . . . . . . . . . . . 58

4 Summary and Conclusions $\quad 61$

4.1 Recommendation for Future Work . . . . . . . . . . . . . . . 62

A Appendix: Overall Plots $\quad 63$

$\begin{array}{ll}\text { B Appendix: Near Term Plots } & 77\end{array}$

$\begin{array}{ll}\text { C Appendix: Mid Term Plots } & 90\end{array}$

D Appendix: Far Term Plots 113

$\begin{array}{ll}\text { Bibliography } & 128\end{array}$ 


\section{List of Tables}

2.1 Baseline RPS . . . . . . . . . . . . . . . . . . . 10 


\section{Acknowledgment}

The work performed at Virginia Tech is supported by Aerojet through the National Institute of Aerospace (NIA) subcontract X05-9005-VT. The Aerojet prime contract NNC05CB09C, administered by the NASA Glenn Research Center under the Exploration Systems Research and Technology In-STEP program 


\section{List of Figures}

1.1 Hall Thruster Efficiency Model . . . . . . . . . . . . . . . . . 4

2.1 Initial orbit versus system masses for RPC Baseline . . . . . . . . . 11

2.2 Initial orbit versus payload percentage for RPC Baseline . . . . . . 11

3.1 Payload Mass . . . . . . . . . . . . . . . . . . . . . . . 13

3.2 Payload Mass . . . . . . . . . . . . . . . . . . . . . . . 13

3.3 Overall Constrained Initial Mass . . . . . . . . . . . . . . . . . 14

3.4 Overall Minimum Boundary Constrained Initial Mass . . . . . . . . 15

3.5 Power vs. Total Time of Flight for a Range of Specific Impulses, Overall 16

3.6 Total Time of Flight as a Function of Power, Overall . . . . . . . 16

3.7 Initial Mass vs. Total Time of Flight, Overall _ . . . . . . . . 17

3.8 Initial Mass vs. Payload Mass per Day, Overall . . . . . . . . . . 18

3.9 Specific Impulse vs. Transfer Time, Overall . . . . . . . . . . . . . 18

3.10 Initial Mass Diminishing Returns as a Function of Specific Impulse, Overall . . . . . . . . . . . . . . . . . . . . . . . 19

3.11 Transfer Time as a Function of Specific Impulse for the Maximum Optimum, Overall . . . . . . . . . . . . . . . 20

3.12 Initial Mass Diminishing Returns as a Function of Power, Overall . . 21

3.13 Payload Masses for all Specific Impulses and Power Levels, Near Term 22

3.14 Constrained Initial Mass as a Function of Power . . . . . . . . . . 24

3.15 Constrained Payload Mass _ . . . . . . . . . . . . . 25

3.16 Maximum payload mass for each specific impulse . . . . . . . . 26

3.17 Specific impulse versus payload mass . . . . . . . . . . . . 27 
3.18 Power versus payload mass . . . . . . . . . . . . . . 28

3.19 Initial Mass vs. Transfer Time, Delta II example . . . . . . . . . . . . 29

3.20 Payload Mass per Day as a Function of Initial Mass . . . . . . . . . . 29

3.21 Initial Mass Diminishing Returns as a Function of Specific Impulse . 30

3.22 Transfer Time as a Function of Specific Impulse for the Maximum

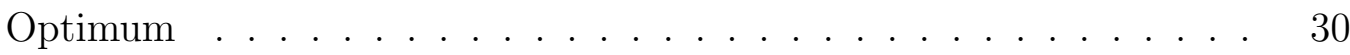

3.23 Initial Mass Diminishing Returns as a Function of Power . . . . . . . 31

3.24 Initial Mass vs. Transfer Time, Delta II example . . . . . . . . . . . . 31

3.25 Initial Mass vs. Payload Mass per Day, Delta II example . . . . . . . 32

3.26 Constrained Initial Mass . . . . . . . . . . . . . . . . . . 33

3.27 Power vs. Total Time of Flight for a Range of Specific Impulse, Midterm 34

3.28 Total Time of Flight as a Function of Power . . . . . . . . . . . 35

3.29 Initial Mass vs. Total Time of Flight, Midterm . . . . . . . . . . . 36

3.30 Initial Mass vs. Payload Mass per Day, Midterm . . . . . . . . . . . 37

3.31 Maximum Payload Mass for Each Specific Impulse, Midterm . . . . . 38

3.32 Specific Impulse vs. Transfer Time, Midterm . . . . . . . . . . . . . 39

3.33 Initial Mass Diminishing Returns as a Function of Specific Impulse . 40

3.34 Transfer Time as a Function of Specific Impulse for the Maximum

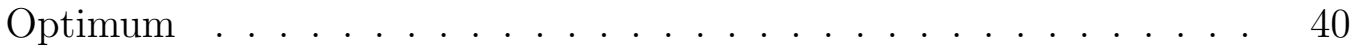

3.35 Initial Mass Diminishing Returns as a Function of Power . . . . . . 41

3.36 Specific impulse vs. Optimum Payload Mass . . . . . . . . . . . . . 41

3.37 Constrained Initial Mass . . . . . . . . . . . . . . . . . . . 42

3.38 Power vs. Total Time of Flight for a range of Specific Impulse, Far Term 43

3.39 Total Time of Flight as a Function of Power . . . . . . . . . . . 44

3.40 Initial Mass vs. Total Time of Flight, Far Term . . . . . . . . . . 45

3.41 Initial Mass vs. Payload Mass per Day, Far Term . . . . . . . . . 46

3.42 Maximum Payload Mass for Each Specific Impulse . . . . . . . . . 47

3.43 Specific Impulse vs. Transfer Time, Far Term . . . . . . . . . . 47

3.44 Initial Mass Diminishing Returns as a Function of Power . . . . . . 48

3.45 Initial Mass Diminishing Returns as a Function of Specific Impulse . 48 
3.46 Transfer Time as a Function of Specific Impulse for the Maximum

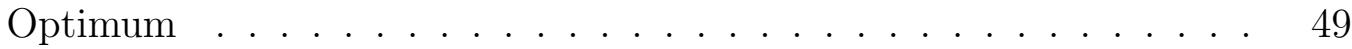

3.47 Specific impulse vs. Optimum Payload Mass . . . . . . . . . . . . 49

3.48 Transfer Time as a Function of Specific Impulse . . . . . . . . . . 51

3.49 Transfer Time as a Function of Power . . . . . . . . . . . . . . 51

3.50 Payload Ratio Percentage as a Function of Initial Mass . . . . . . . . 52

3.51 Payload Mass Percentage as a Function of Initial Orbit Altitude . . . 53

3.52 Payload Mass as a Function of Initial Orbit Altitude . . . . . . . . 54

3.53 Payload Mass as a Function of Initial Mass . . . . . . . . . . . . . 54

3.54 Constrained Initial Mass for Near Term Technology . . . . . . . . 55

3.55 Constrained Initial Mass for Mid Term Technology . . . . . . . . 55

3.56 Constrained Initial Mass for Far Term Technology . . . . . . . . . 56

3.57 Maximum Payload Mass for Near Term Technology . . . . . . . . . 56

3.58 Maximum Payload Mass for Mid Term Technology . . . . . . . . . 57

3.59 Maximum Payload Mass for Far Term Technology . . . . . . . . . 57

3.60 RLEP Schedule Comparison . . . . . . . . . . . . . . . . . . 60

A.1 Power vs. Total Time of Flight, Overall . . . . . . . . . . . . . 63

A.2 Power vs. Total Time of Flight, Overall . . . . . . . . . . . . . . 64

A.3 Initial Mass vs. Total Time of Flight, Overall . . . . . . . . . . 65

A.4 Initial Mass vs. Total Time of Flight, Overall . . . . . . . . . 66

A.5 Initial Mass vs. Payload Mass per Day, Overall . . . . . . . . . . 67

A.6 Initial Mass vs. Payload Mass per Day, Overall . . . . . . . . . . . 68

B.1 Initial Mass versus Payload Mass _ . . . . . . . . . . . . . 78

B.2 Initial Mass versus Payload Mass _ . . . . . . . . . . . . . . . 79

B.3 Initial Mass vs. Payload Mass per Day . . . . . . . . . . . . . . 80

B.4 Initial Mass vs. Payload Mass per Day . . . . . . . . . . . . . . 81

B.5 Initial Mass vs. Payload Mass per Day . . . . . . . . . . . . . 82

B.6 Initial Mass vs. Payload Mass per Day . . . . . . . . . . . . . 83

C.1 Power vs. Total Time of Flight $\ldots \ldots \ldots \ldots \ldots$ 
C.2 Power vs. Total Time of Flight _. . . . . . . . . . . . . . . . 91

C.3 Initial Mass vs. Total Time of Flight . . . . . . . . . . . . . . . 92

C.4 Initial Mass vs. Total Time of Flight . . . . . . . . . . . . . 93

C.5 Initial Mass vs. Payload Delivered Per Day . . . . . . . . . . . . . 94

C.6 Initial Mass vs. Payload Delivered Per Day . . . . . . . . . . . . . 95

D.1 Initial Mass versus Payload Mass . . . . . . . . . . . . . . . . 113

D.2 Initial Mass versus Payload Mass . . . . . . . . . . . . . . . 114

D.3 Initial Mass versus Payload Mass . . . . . . . . . . . . . . . 115

D.4 Initial Mass vs. Payload Mass per Day . . . . . . . . . . . . . . 116

D.5 Initial Mass vs. Payload Mass per Day . . . . . . . . . . . . . . 117

D.6 Initial Mass vs. Payload Mass per Day _ . . . . . . . . . . . . . 118

D.7 Initial Mass vs. Payload Mass per Day . . . . . . . . . . . . . . 119

D.8 Initial Mass vs. Payload Mass per Day . . . . . . . . . . . . . 120

D.9 Initial Mass vs. Payload Mass per Day . . . . . . . . . . . . . . . 121

D.10 Power vs. Transfer Time . . . . . . . . . . . . . . . . . . . . . 122

D.11 Power vs. Transfer Time . . . . . . . . . . . . . . . . . . . . . 123

D.12 Power vs. Transfer Time . . . . . . . . . . . . . . . . . . . 124 


\section{List of Symbols}

$$
\begin{array}{ll}
\Delta V_{\text {chem }} & =\text { delta velocity for chemical propulsion mission }\left(\frac{m}{s}\right) \\
\Delta V_{1} & =\text { delta velocity for chemical phase }\left(\frac{m}{s}\right) \\
\Delta V_{2} & =\text { delta velocity for electric phase }\left(\frac{m}{s}\right) \\
\Delta V_{2 e f f} & =\Delta V_{\text {chem }}-\Delta V_{1}\left(\frac{m}{s}\right) \\
\eta_{v} & =\text { mission planning efficiency } \\
c_{1} & =\text { effective chemical thruster exhaust velocity }\left(\frac{m}{s}\right) \\
c_{2} & =\text { effective electric thruster exhaust velocity }\left(\frac{m}{s}\right) \\
I_{s p} & =\text { specific impulse (sec) } \\
v_{c h} & =\text { system's characteristic velocity }\left(\frac{m}{s}\right) \\
I_{s}^{*} & =\text { optimum specific impulse (sec) either fixed or variable thrust } \\
g & =\text { acceleration due to gravity }\left(\frac{m}{s e c^{2}}\right) \\
G & =\text { gravitational constant }\left(\frac{N m^{2}}{\mathrm{~kg}^{2}}\right) \\
\theta & =\text { angle between spacecraft and line of nodes (radians) } \\
M & =\text { spacecraft mass }(\mathrm{kg}) \\
M_{o} & =\text { planet mass (kg) } \\
F_{\theta} & =\text { thrust along the flight path }(\mathrm{N}) \\
T & =\text { thrust (N) } \\
P & =\text { power (Watts or } \mathrm{kW}) \\
\eta & =\text { electric propulsion thruster efficiency } \\
\dot{m} & =\text { propellant mass flow rate }\left(\frac{\mathrm{kg}}{\mathrm{s}}\right)
\end{array}
$$




\title{
Nomenclature
}

\author{
HET Hall Effect Thruster \\ RLEP Robotic Lunar Exploration Proram \\ RPS Robotic Precursor System \\ ERPS Electric Robotic Precursor System \\ LEO Low Earth Orbit \\ GTO Geosynchronous Transfer Orbit \\ LLO Low Lunar Orbit \\ GEO Geosynchronous Earth Orbit \\ SEP Solar Electric Propulsion \\ TLI Translunar Injection \\ LOX Liquid oxygen \\ ADCS Attitude Determination and Control System \\ GNC Guidance Navigation and Control \\ TCS Thruster Control System \\ PCS Propellant Control System \\ TCU Thruster Control Unit \\ PPU Power Processing Unit \\ LRO Lunar Reconnaissance Orbiter
}




\section{Chapter 1}

\section{Introduction and Background}

The space exploration program laid out in 2004[1] specifies objectives for lunar exploration. Among these are to undertake activities that prepare for and enable future human exploration, including a series of robotic missions, and the use of these exploration activities to develop new approaches, technologies, and systems for the future[2][3].

With these overarching goals in mind, there is a need to deliver non-time critical payloads in the near future for Robotic Lunar Exploration Program (RLEP) missions and for human explorations of the Moon. A direct drive Solar Electric Propulsion (SEP) system may be advantageous for such a purpose as it has the potential to deliver more payload to the lunar surface than chemical propulsion and reduce the mission cost.

This paper investigates the concept of solar electric propulsion for robotic lunar missions using high power Hall thrusters. A trade study is carried out to understand the relationships between payload mass delivered, electric propulsion power, and trip time. A comparison is made to a chemical propulsion system with $\mathrm{LOX} / \mathrm{H}_{2}$ as the baseline. In this study, the system will be called a Robotic Precursor System (RPS) and the electrically propelled version will be called the Electric Robotic Precursor System (ERPS). The major differences between the two systems are presented in terms of the two possible sets of payloads (one for the chemically propelled probe and one for the electrically propelled probe) and trip times. 
Both orbiters and landers are mentioned as possible missions. Several launch systems are mentioned that are capable of delivering to Low Lunar Orbit (LLO), thus giving a rough range of future mission sizes being considered; estimates range from $274 \mathrm{~kg}$ to $6598 \mathrm{~kg}$ delivered[2]. For example, the Lunar Reconnaissance Orbiter (LRO) which is currently being designed, is slated to be approximately $1000 \mathrm{~kg}$ $1450 \mathrm{~kg}[4]$. A second planned RLEP mission is a lunar lander[5] launching as early as 2010. Available lander mass estimates range from approximately $250 \mathrm{~kg}$ to $550 \mathrm{~kg}$ delivered to the surface.

It has been previously shown that electrically propelled transfer vehicles provide mass advantages for Earth orbit raising missions when combined with chemical propulsion[6][7]. It has also been shown that Hall thrusters in the range of $10 \mathrm{~kW}$ using direct drive can propel small spacecraft to various small bodies within the solar system[8]. Another study investigated a solar electric propulsion option for cargo delivery to the lunar surface[9]. In that study a 3000 second specific impulse cargo tug was able to deliver twice that of a conventional chemical propulsion system. This study will investigate electrically propelled spacecraft using a direct drive Hall thruster for lunar missions for a much larger range of specific impulses and thruster power levels as applied to RLEP missions (up to 10,000 kg). To ensure a full mapping of the parameter space, the specific impulse is varied from 1000 seconds to 28,500 seconds and the thruster power level is varied from $10 \mathrm{~kW}$ to $200 \mathrm{~kW}$.

\section{$1.1 \quad$ Approach}

\subsubsection{Mission Definition}

This study assumes certain parameters for the RPS and ERPS future vehicles and missions. The first RLEP mission considered will begin in 2010 and the missions will continue indefinitely. The RPS and ERPS launch into Geosynchronous Transfer Orbit (GTO), defined as $185 \mathrm{~km} \times 35,786 \mathrm{~km}$ at an inclination of $28.5^{\circ}$. The ending orbit is assumed to be a circular Low Lunar Orbit (LLO) at an altitude of $100 \mathrm{~km}$. 
The launch vehicle used here for the near term analysis is the Boeing Delta II 7925H10, which can carry a payload of approximately $2100 \mathrm{~kg}[10]$ to GTO at an inclination of $28.7^{\circ}$; the assumption is made that there is no difference for this small inclination difference. For the mid term and far term analyses, no assumption is made on the launch vehicle. The main requirement is that the transfer time be less than one year.

\subsubsection{High-Thrust and Low-Thrust Trajectories}

In this study, the trajectories considered for the chemical propulsion based RPS use a Hohmann transfer and calculated using the commercial software Satellite Toolkit (STK). The trajectories considered for ERPS consist of a low-thrust outward spiral orbit with constant thrusting and a downward spiral into lunar orbit. The radial and tangential orbital equations of motions follow[11].

$$
\begin{gathered}
M\left(\ddot{r}-r \dot{\theta}^{2}\right)=-m \frac{G M_{o}}{r^{2}} \\
M(r \ddot{\theta}+2 \dot{r} \dot{\theta})=F_{\theta}
\end{gathered}
$$

The basic equations describing the electric propulsion for thrust and mass flow rate follow $[12]$.

$$
\begin{gathered}
T=\frac{2 \eta P_{0}}{g I_{s p}} \\
\dot{m}=\frac{T}{g I_{s p}}
\end{gathered}
$$

For the ERPS the low-thrust Earth escape spiral is computed using orbital averaging, then ballistic coast to lunar sphere of influence is computed using two-body motion. Next, the low-thrust lunar capture spiral is computed using "backward" integration with orbital averaging and precise numerical integration. The transfer time was calculated using numerical integration for the low-thrust escape and capture spirals and the coast transfer time is calculated using two-body motion. 


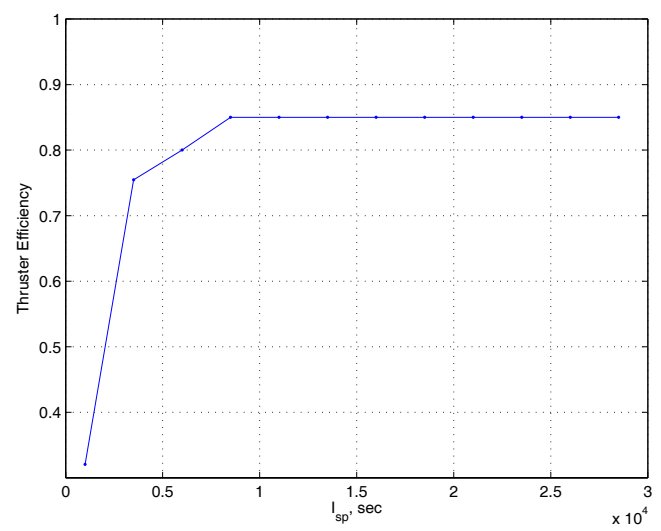

Figure 1.1: Hall Thruster Efficiency Model

The efficiency of the Hall thruster, $\eta$, is based on empirical data from testing of a $50 \mathrm{~kW}$ laboratory model[13]. The model used relates the efficiency of the thruster to the specific impulse; since the data only has a range to a specific impulse of 3000 sec, an assumption is made that after this point it rises to an asymptote leveling off around 0.85 based on other analyses of Hall thrusters[14], which can be seen in Figure 1.1. 


\section{Chapter 2}

\section{Mass Models}

\subsection{Robotic Precursor System Mass Model}

The Lunar Reconnaissance Orbiter (LRO) is used as the model for future RPS systems. LRO has an estimated spacecraft dry mass of $1000 \mathrm{~kg}$, bus mass of $495 \mathrm{~kg}$, $405 \mathrm{~kg}$ of propellant, and $100 \mathrm{~kg}$ of payload. Therefore, approximately $83 \%$ of the LRO spacecraft dry mass is bus. This study uses that factor by assuming that $83 \%$ of the RPS dry mass is bus and the rest is payload mass.

Equation 2.1 defines the propellant mass as the amount launched into the initial orbit by the launch vehicle minus the mass in LLO. Equation 2.2 defines the bus mass as $83 \%$ of the dry mass, which is the spacecraft mass without propellant and shown here as the mass in LLO. Equation 2.3 defines the payload mass as the mass in LLO minus the bus mass.

$$
\begin{gathered}
M_{\text {Propellant }}=M_{L V}-M_{L L O} \\
M_{\text {Bus }}=0.83 \times M_{L L O} \\
M_{\text {Payload }}=M_{L L O}-M_{\text {Bus }}
\end{gathered}
$$




\subsection{Electric Robotic Precursor System Mass Model}

\subsubsection{Spacecraft Model}

The spacecraft mass model[15] is defined as a payload, the SEP stage, and the propellant, as shown in equation 2.4. The SEP stage is defined to have several major subsystems, as shown in equation 2.5: Attitude Determination and Control System (ADCS), Guidance Navigation and Control (GNC), Power, Propulsion, Structure, Telecommunications System (Comm), and Thermal Management System (Thermal). The propellant is determined using a trajectory code and the payload is defined as the launch vehicle capability minus the propellant and SEP stage, as shown in equation 2.6 .

$$
\begin{gathered}
M_{S / C}=M_{\text {Payload }}+M_{\text {Propellant }}+M_{S E P} \\
M_{S E P}=M_{A D C S}+M_{G N C}+M_{\text {Power }}+M_{\text {Propulsion }}+M_{\text {Comm }}+M_{\text {Thermal }} \\
M_{\text {Payload }}=M_{L \text { Capability }}-\left(M_{S E P}+M_{\text {Propellant }}\right)
\end{gathered}
$$

\subsubsection{Attitude Determination and Control System}

The attitude control is assumed to be provided by vectoring the thrust from the electric thrusters using a gimbal system, which is the only defined component of the attitude determination and control (ADCS) system. The gimbal system is defined as $35 \%$ of the electric thruster and the ADCS mass calculation is shown in Equation 2.7 .

$$
M_{A D C S}=0.35 \times M_{\text {Thruster }}
$$




\subsubsection{Guidance Navigation and Control System}

The GNC is assumed to have a fixed mass of $1 \mathrm{~kg}$. This assumption is made on the basis of historical information.[15]. This is typically a small mass and will not impact the overall results significantly.

\subsubsection{Power System}

The power system is defined to use solar arrays and there is no consideration of power storage. The equation for calculating the power system mass is shown in Equation 2.8 and includes miscellaneous mass to account for some cabling and drive assemblies. The solar array mass is calculated by dividing the required output power by the solar array specific power as shown in Equation 2.9.

$$
\begin{gathered}
M_{\text {Power }}=1.054 \times M_{\text {Array }} \\
M_{\text {Array }}=\frac{P_{\text {out }}}{P_{\text {Specific Power }}}
\end{gathered}
$$

\subsubsection{Propulsion System}

The mass of the propulsion system is defined to be the electric thrusters plus the thruster control system (TCS), the propellant control system (PCS), and propellant tank masses as shown in Equation2.10. It is assumed there is only one electric thruster and therefore the Hall thruster mass is calculated as a single unit based on a defined thruster input power as shown in Equation 2.11. The thruster control system consists of the direct drive electronics. The TCS is comprised of a single direct drive thruster control unit[16] and the mass of this unit is calculated as a function of thruster input power as shown in Equation 2.12. This equation is generated by the assumption that a direct drive thruster control unit (TCU) would be $35 \%$ of the mass of a conventional power processing unit. The propellant control system is fixed at $4 \mathrm{~kg}$ based on the currently available technology and the assumption that flow rates would not increase sufficiently to change component masses. The propellant tank mass is calculated using 
Equation 2.13 which is a function of the propellant mass generated by iterations of the trajectory code.

$$
\begin{gathered}
M_{\text {Propulsion }}=M_{\text {Thruster }}+M_{T C S}+M_{P C S} \\
M_{\text {Thruster }}=2.5 \times P_{\text {in }} \\
M_{\text {TCS }}=1.379 \times\left(P_{\text {in }}\right)^{0.73} \\
M_{\text {Tank }}=0.035 \times M_{\text {Propellant }}
\end{gathered}
$$

\subsubsection{Structural System}

The spacecraft structural system is comprised of a single truss with cabling for electrical and data service. The calculation of the structural system mass is shown in Equation 2.14. The truss length is calculated using Equation 2.15 which is a function of the thruster input power $\left(P_{i n}\right)$, solar areal power density $\left(P_{\text {Solar }}\right.$ assumed to be constant at $\left.1300 \frac{W}{m^{2}}\right)$, the TCU efficiency $\left(\varepsilon_{T C U}\right)$, the solar array efficiency $\left(\varepsilon_{\text {Array }}\right)$, and the solar array aspect ratio $\left(A R_{\text {Solar }}\right)$. The truss mass is then calculated using Equation 2.16 which is a function of truss length and truss specific mass, $\alpha_{\text {Truss }}$. The cabling mass is calculated using Equation 2.17 which is a function of truss length and cable specific mass, $\alpha_{\text {Cabling }}$.

$$
\begin{gathered}
M_{\text {Structure }}=M_{\text {Truss }}+M_{\text {Cabling }} \\
L_{\text {Truss }}=0.75 \times\left(\frac{1000 \times P_{\text {in }}}{P_{\text {Solar }} \times \varepsilon_{\text {TCU }} \times \varepsilon_{\text {Array }}}\right)^{0.5} \times\left(\frac{1}{A R_{\text {Solar }}}\right)^{-0.5} \\
M_{\text {Truss }}=L_{\text {Truss }} \times \alpha_{\text {Truss }} \\
M_{\text {Cabling }}=L_{\text {Truss }} \times \alpha_{\text {Cabling }}
\end{gathered}
$$




\subsubsection{Telecommunications System}

The telecommunications systems is assumed to be fixed at $40 \mathrm{~kg}$. This assumption is made on the basis of historical information.[15]. This is typically a small mass and will not impact the overall results significantly.

\subsubsection{Thermal Management System}

The thermal management system is defined to be equal to the radiator mass as shown in Equation 2.18. The radiator mass is calculated using Equation 2.19 which is a function of thruster input power $\left(P_{\text {in }}\right)$, radiator specific mass $\left(\alpha_{\text {Radiator }}\right)$, and TCU efficiency $\left(\varepsilon_{T C U}\right)$.

$$
\begin{gathered}
M_{\text {Thermal }}=M_{\text {Radiator }} \\
M_{\text {Radiator }}=P_{\text {in }} \times \alpha_{\text {Radiator }} \times \frac{1-\varepsilon_{T C U}}{\varepsilon_{T C U}}
\end{gathered}
$$


Table 2.1: Baseline RPS

\begin{tabular}{|l|c|}
\hline Mission $\Delta V$ & $1530 \frac{\mathrm{m}}{\mathrm{s}}$ \\
Specific Impulse & $450 \mathrm{~s}$ \\
Initial Mass & $2100 \mathrm{~kg}$ \\
Fuel Mass & $615.1 \mathrm{~kg}$ \\
Bus Mass & $1232.5 \mathrm{~kg}$ \\
Payload Mass & $252.4 \mathrm{~kg}$ \\
Payload Mass Percentage & $12.0 \%$ \\
Flight Time & 4.08 days \\
\hline
\end{tabular}

\subsection{Baseline Robotic Precursor System}

The baseline RPS uses a $\mathrm{LOX} / \mathrm{LH}_{2}$ chemical propulsion system, estimated here at a $450 \mathrm{sec}$ specific impulse[17]. The spacecraft is launched by a Delta II into a $185 \mathrm{~km}$ $\times 35,786 \mathrm{~km}$ orbit. Again, the spacecraft bus is estimated as $83 \%$ of the dry mass.

The initial orbit for the baseline is varied for a range of circular orbits, to see if there is any obvious advantage. The payload mass for the baseline initial orbit is $252 \mathrm{~kg}$; Figure 2.1 shows that there is no clear gain; in fact there is a loss when compared to the GTO orbit selected. The payload ratio percentage is $12.0 \%$ for the baseline initial orbit. Figure 2.2 presents the payload mass percentage of the initial mass, which is lower than for the baseline GTO orbit. Since this is the case, the baseline GTO orbit will be used. 


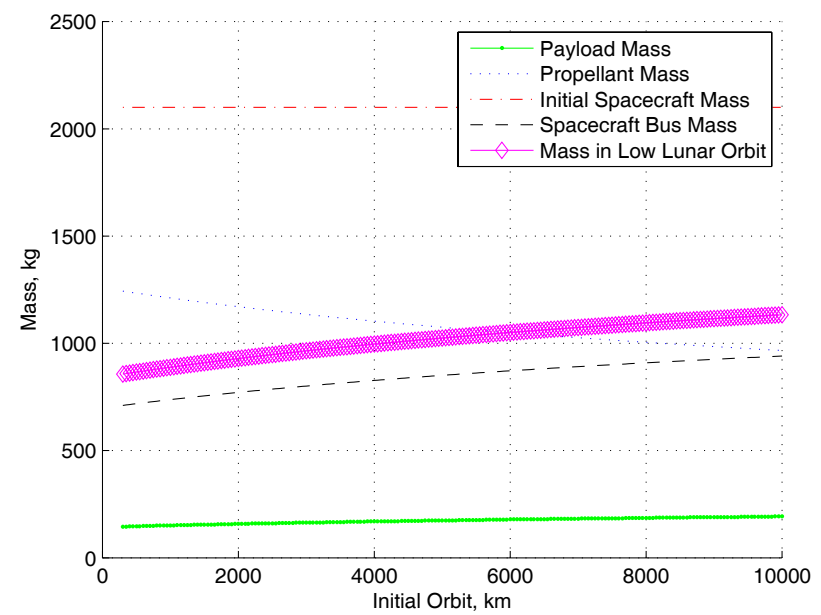

Figure 2.1: Initial orbit versus system masses for RPC Baseline

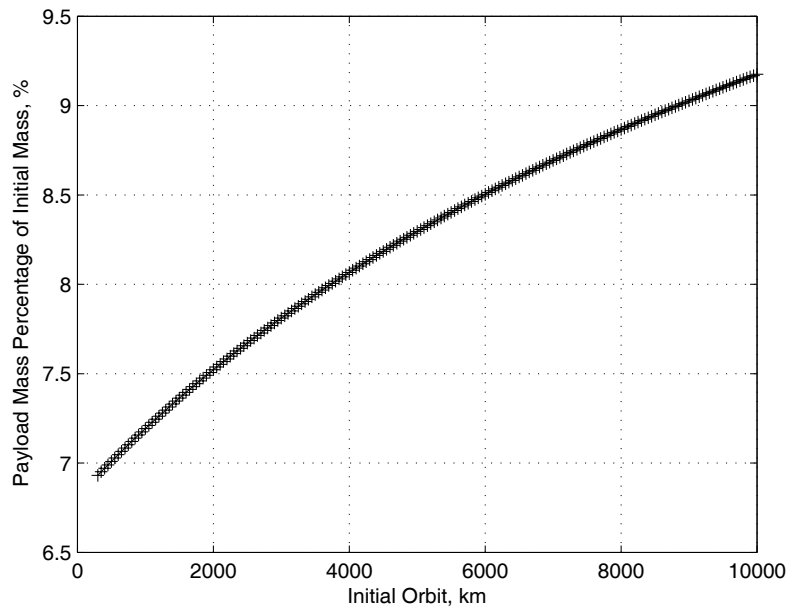

Figure 2.2: Initial orbit versus payload percentage for RPC Baseline 


\section{Chapter 3}

\section{Electric Robotic Precursor System}

\subsection{Overview}

The main goal of this investigation is to determine the relationships between inputs of system power, initial mass, and thruster specific impulse to outputs such as payload mass in LLO, transfer time, and propellant mass. To this end, approximately 18,000 cases were run varying the initial mass $(500 \mathrm{~kg}$ to $10,000 \mathrm{~kg}$ ), Hall thruster power (10 kW to $200 \mathrm{~kW}$ ), and specific impulse of the Hall thruster (1000 sec to 28,500 sec).

This section will highlight the results to gain a big picture vantage. First it is helpful to see how the payload mass behaves as a function of the inputs. Figure 3.1 shows a series of surfaces each with a constant power, with the colorbar representing the payload mass. Low power levels are on the left with the transfer time increasing to the right as the power increases. Figure 3.2 shows the same results but with each surface at a constant specific impulse, with the colorbar representing the payload mass. In general the power has a large effect on the payload mass while the specific impulse has less of an effect.

Next, it is useful to examine the required initial mass for each power level and specific impulse. There are two main requirements on these cases: that a positive amount of payload must be delivered (a physical requirement) and the transfer time must not exceed one year. The first requirement will drive the bound of minimum initial mass and the transfer time requirement will drive the maximum initial mass 


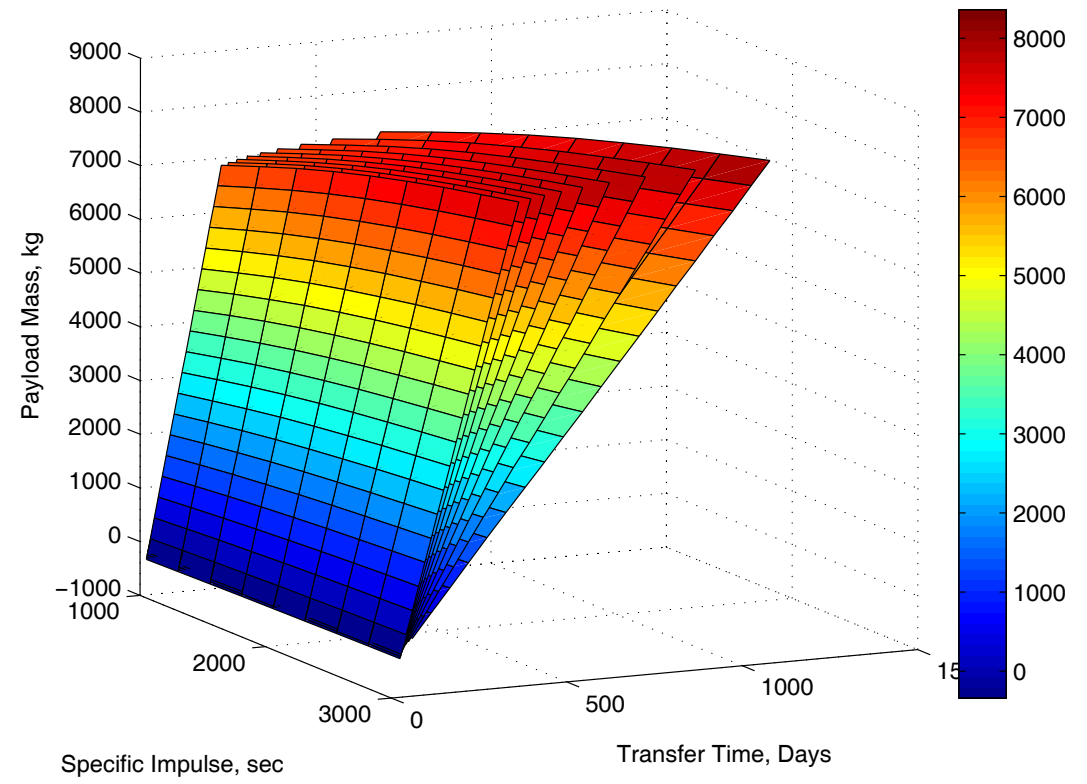

Figure 3.1: Payload Mass

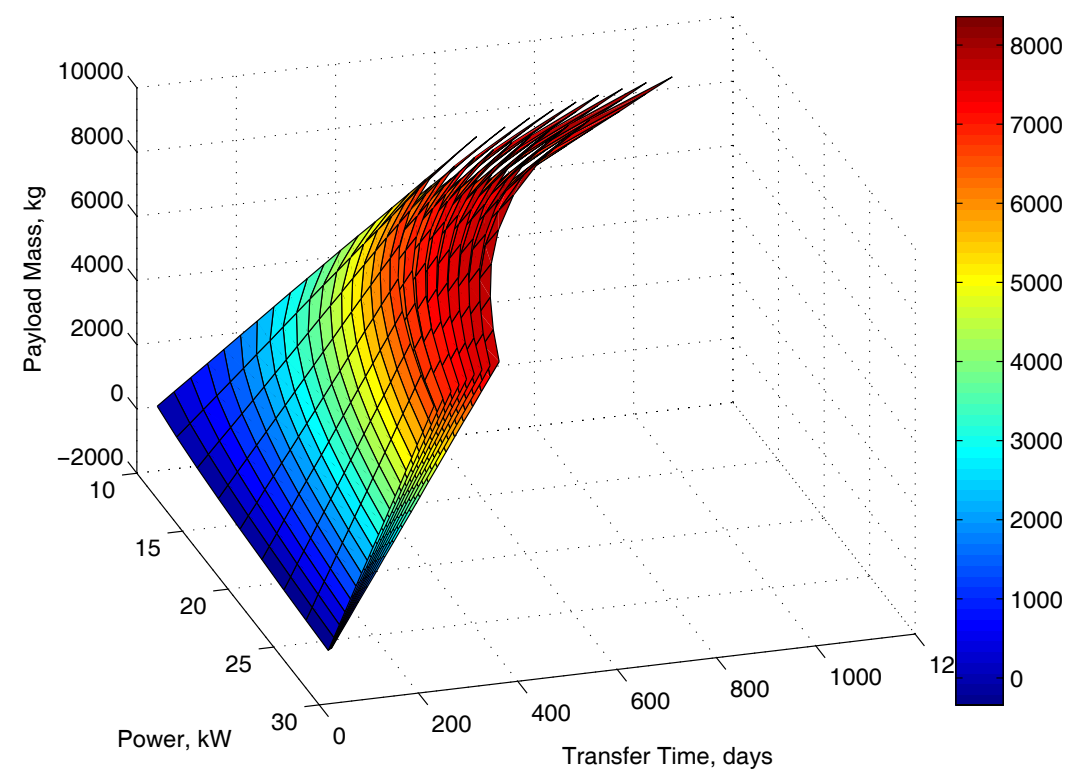

Figure 3.2: Payload Mass 


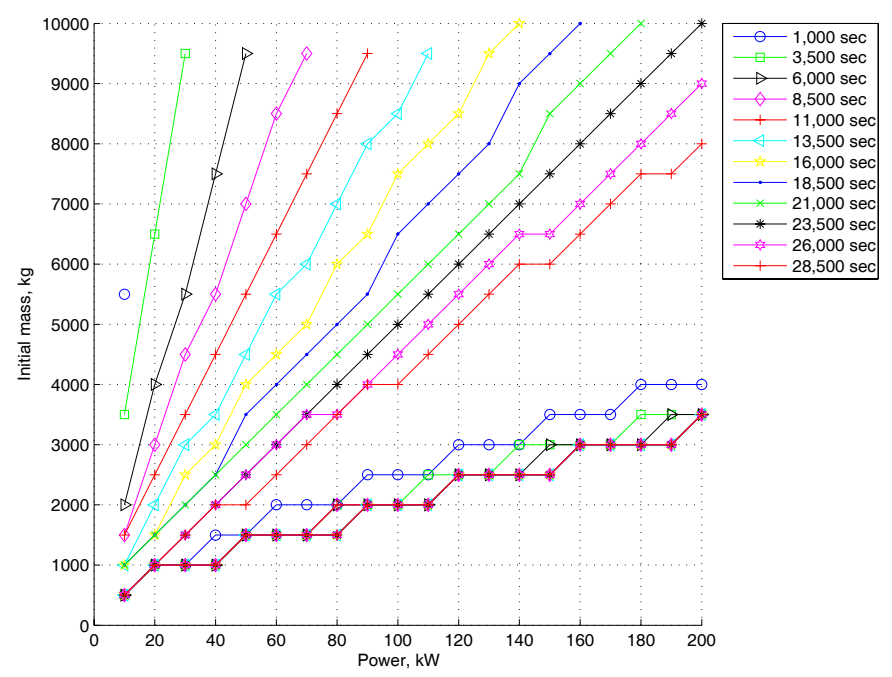

Figure 3.3: Overall Constrained Initial Mass

boundary. This information is particularly helpful in determining what combinations of power and specific impulse will allow successful completion of the mission given a launch vehicle mass constraint.

A plot of the required initial mass for each power level and specific impulse is presented in Figure 3.3. The maximum initial mass lines end at 10,000 kg initial mass, where the investigated range ends. To the right, up to $10,000 \mathrm{~kg}$, the transfer time does not constrain the initial mass. The region between the two sets of lines indicates a combination of initial mass, specific impulse, and power level that will result in delivery of a positive payload mass in under one year to lunar orbit. It is somewhat difficult to see from this plot what the requirements on the initial mass are for the low power levels. To aid this discrimination, the minimum boundary has been separated out in Figure 3.4. In a another section we will discuss the low power levels with more resolution.

Now we move to an investigation of the relationship between power and trip time, which is a main concern for this analysis. Figure 3.5 shows power versus the maximum and minimum total transfer time (within the range of initial masses) for the specific impulse range specified above. Each line has constant specific impulse and is either 


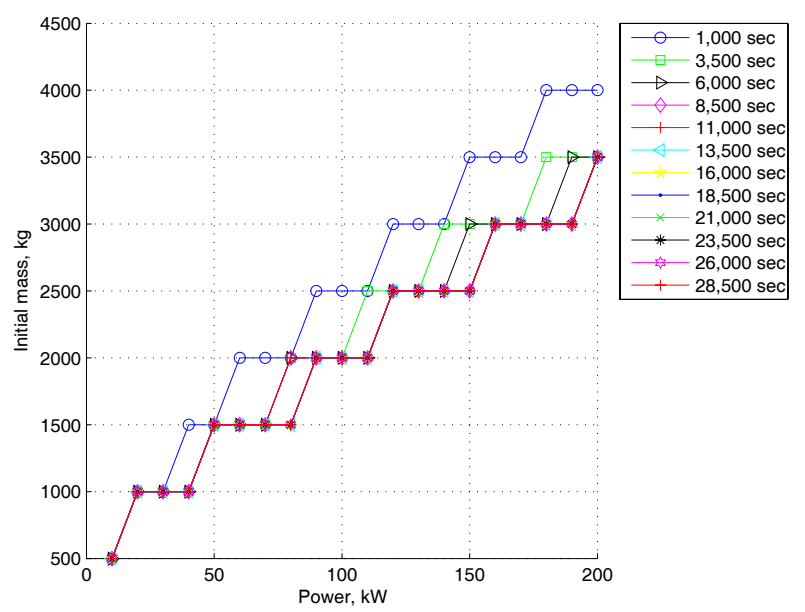

Figure 3.4: Overall Minimum Boundary Constrained Initial Mass

the maximum or minimum transfer time for all the initial masses. The lower grouping consists of all the minimum transfer times and the upper grouping of all the maximum transfer times for the specific impulse range. As expected, the longer flight times are due to the higher specific impulse. Since a large portion results in a long transfer time, longer than the requirement, there may be a restriction on specific impulse and many cases can be immediately thrown out. Most obviously, the transfer time drops dramatically as the power increases; this result is expected as can be seen in Equation 1.3. As power increases so does thrust and therefore the transfer time decreases.

Figure 3.6 is indicative of the transfer time as a function of the power level. The top line is the maximum transfer time, and the bottom line the minimum, for the given specific impulse as a function of power for all the initial masses in the investigated range. This plot shows only four specific impulse levels as a representative set. Figure 3.7 presents the initial mass versus the total transfer time split to show each specific impulse separately. Again, this plot shows only four specific impulse levels as a representative set.

Another viewpoint from which to analyze the data is to look at the payload mass transported per day. By maximizing the amount of payload delivered per day, transfer time (and usually cost along with it) is minimized while payload mass is maximized. 


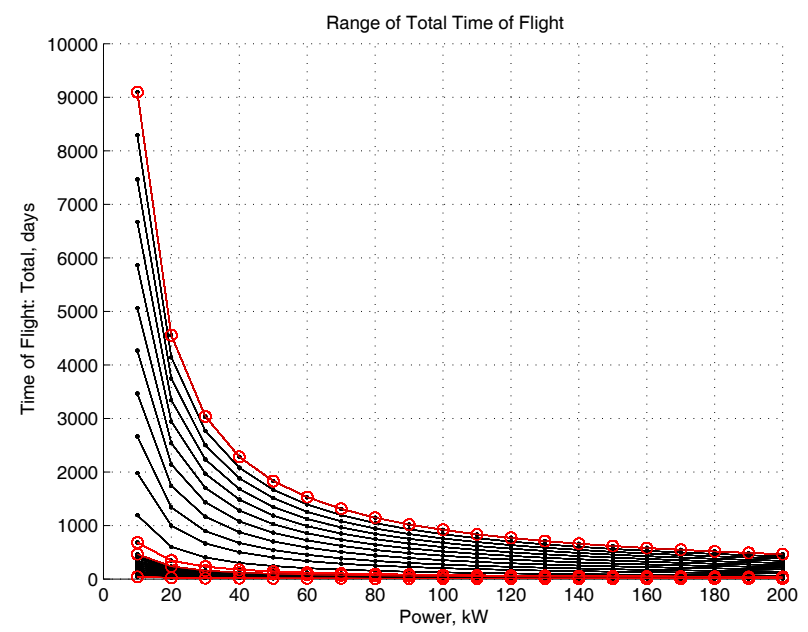

Figure 3.5: Power vs. Total Time of Flight for a Range of Specific Impulses, Overall
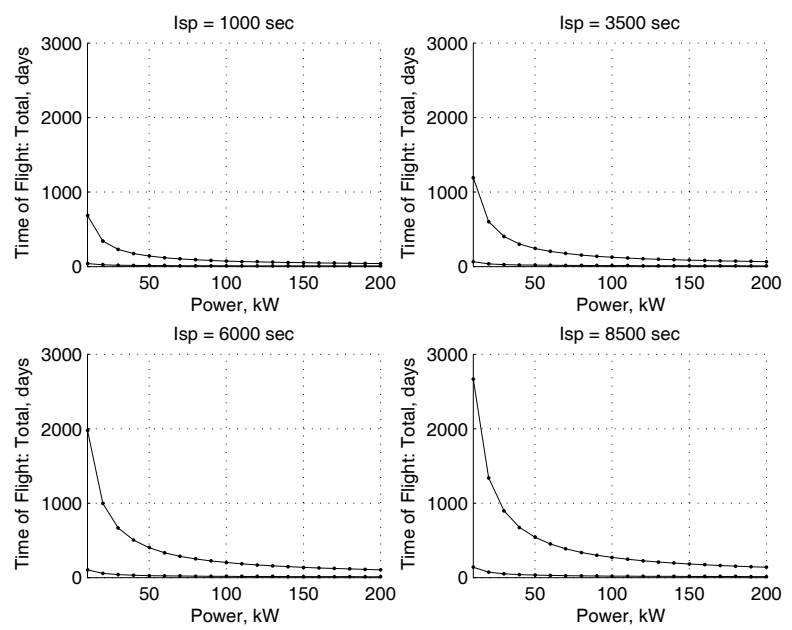

Figure 3.6: Total Time of Flight as a Function of Power, Overall 

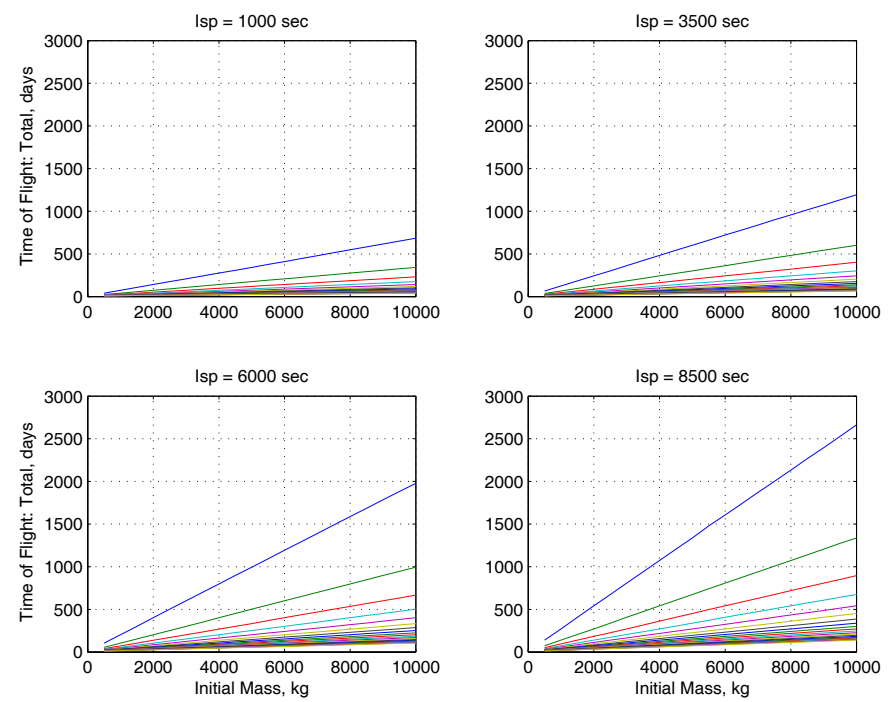

Figure 3.7: Initial Mass vs. Total Time of Flight, Overall

Plotting this quantity versus the initial mass, Figure 3.8 shows that as specific impulse increases the amount of payload mass delivered per day decreases. There are twenty power levels shown in these plots, from $10 \mathrm{~kW}$ to $200 \mathrm{~kW}$ (increments of $10 \mathrm{~kW}$ ) and the bottom curve, the blue curve, corresponds to the lowest power level which increases vertically. As the power increases so does the amount of mass delivered per day. The variation in specific impulse plays a strong role; by increasing this quantity from $1000 \mathrm{sec}$ to $28,500 \mathrm{sec}$, the payload mass delivered to lunar orbit per day drops by about $100 \mathrm{~kg}$. System power has a large effect as well, a variation of $114 \mathrm{~kg} /$ day to $10.5 \mathrm{~kg} /$ day results at an initial mass of 10,000 kg.

To more clearly show the importance of specific impulse, Figure 3.9 presents some selected results of the effect of specific impulse on the transfer time. This figure of plots shows the specific impulse from $1000 \mathrm{sec}$ to $30,000 \mathrm{sec}$; the effect is clearly seen as the transfer time increases by hundreds of days over the range.

\subsubsection{Diminishing Returns}

Looking at the plots of payload mass delivered per day as a function of initial mass brings to light a question: at what point does increasing the initial mass no longer 

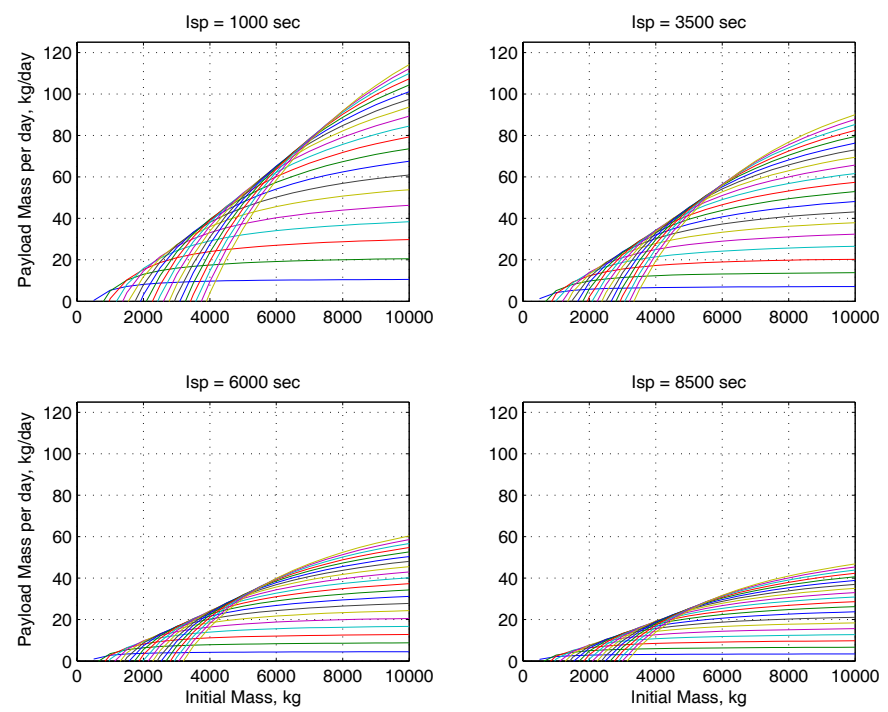

Figure 3.8: Initial Mass vs. Payload Mass per Day, Overall
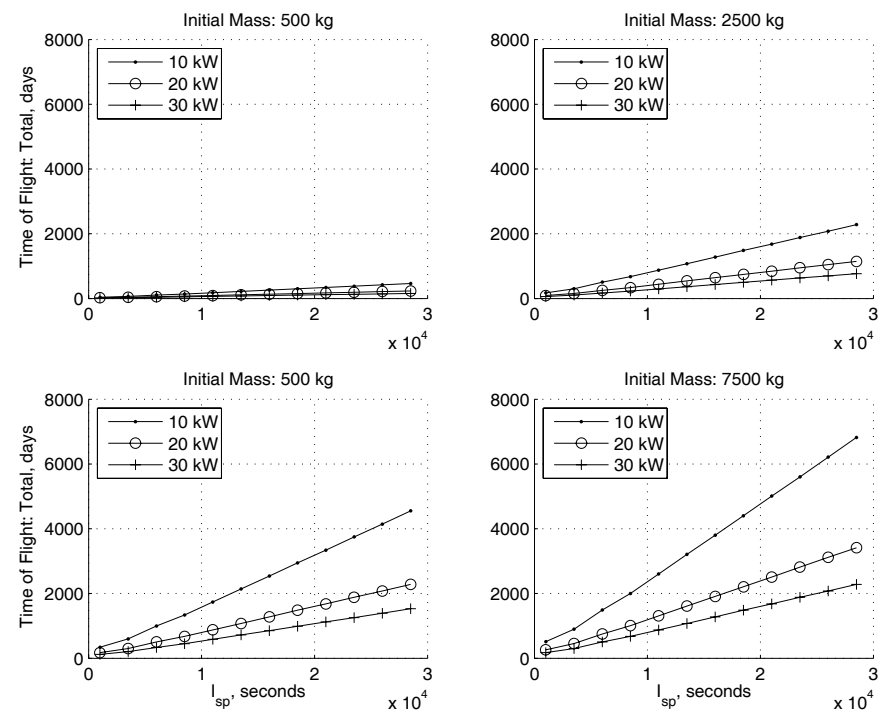

Figure 3.9: Specific Impulse vs. Transfer Time, Overall 


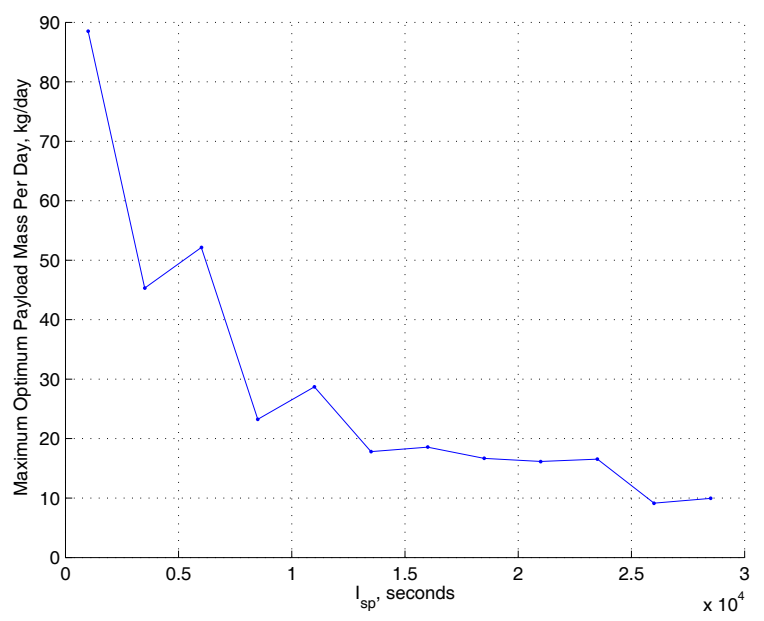

Figure 3.10: Initial Mass Diminishing Returns as a Function of Specific Impulse, Overall

bring an improvement in the result? This can be determined by looking at the rate of change of the result, the payload mass delivered per day in this case. If we look at the rate of change of this quantity, the maximum point is where the greatest change is effected by increasing the initial mass. Beyond this point increasing it further brings about diminished returns. Therefore the optimum initial mass corresponds to that maximum point. Of course, given the step size of the input variables, the determined numeric quantity may not exactly be the optimum.

Figure 3.10 shows the points of diminishing returns as a function of specific impulse. As previously stated, the result decreases with increasing specific impulse since it is inversely proportional to thrust. A couple of the points on this plot do not follow the general trend as closely as might be expected. This is basically due to the fact that the trade space, the investigated ranges of inputs, is constrained. Within this trade space, some of the combinations of inputs do not give a point of diminishing returns.

Figure 3.11 shows the transfer times for the points of maximum optimum payload mass delivered per day, the points of diminishing returns, as a function of specific impulse. Quickly we can eliminate those high specific impulses where the transfer 


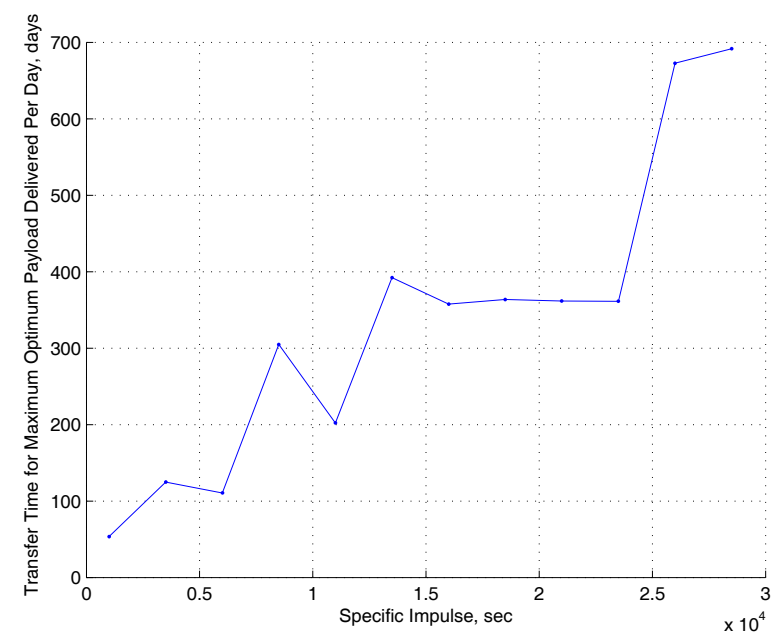

Figure 3.11: Transfer Time as a Function of Specific Impulse for the Maximum Optimum, Overall

time is above one year. This eliminates the 13,500 sec specific impulse, although the next higher four are all under one year. This leaves specific impulses of 1000, 3500, $6000,8500,11,000,16,000,18,500,21,000$, and 23,500 seconds.

Figure 3.12 shows the points of diminishing returns by power level. There are power levels that are not smoothly in line with most of the other results at $130 \mathrm{~kW}$ and $170 \mathrm{~kW}$. The curves of initial mass versus payload mass delivered per day at nearby power levels do not reach a point of diminishing returns, however those are close. It could be expected that by increasing the range of initial masses to $15,000 \mathrm{~kg}$ would reveal a point of diminishing returns. But at $130 \mathrm{~kW}$ and $170 \mathrm{~kW}$ this point comes slightly quicker, and in the investigated range, at around $9000 \mathrm{~kg}$.

The points on this plot where the result is zero indicates a power level where there is no point of diminishing returns. At $180 \mathrm{~kW}$ and $200 \mathrm{~kW}$, where just this case occurs, there is no combination of power levels, specific impulse, and initial mass that brings this about. Looking more closely, an increase in initial mass would bring about this point relatively quickly.

In the next sections we will further investigate these results for near term uses (up to $30 \mathrm{~kW}$ power and $3000 \mathrm{sec}$ specific impulse), mid-term uses (up to $100 \mathrm{~kW}$ power 


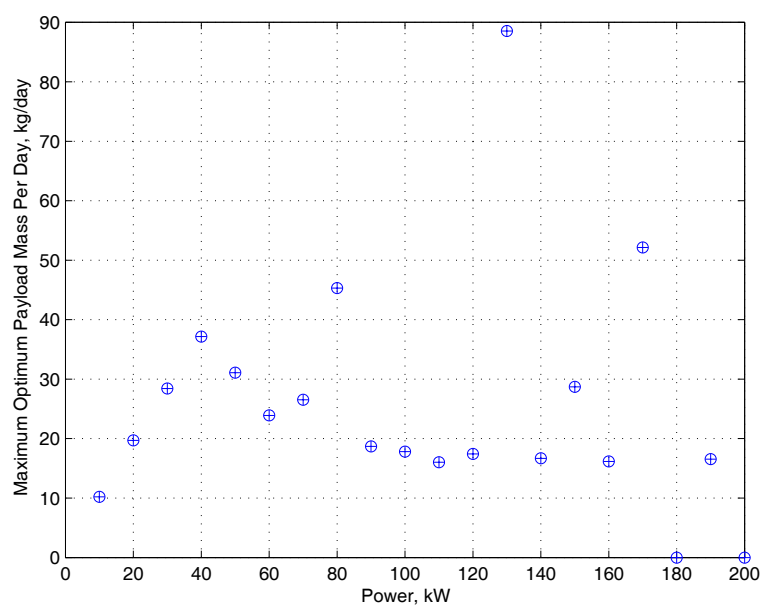

Figure 3.12: Initial Mass Diminishing Returns as a Function of Power, Overall

and 15,000 sec specific impulse) and far-term (up to $200 \mathrm{~kW}$ power and 30,000 sec specific impulse). 


\subsection{Near Term}

Focusing on power levels up to $30 \mathrm{~kW}$ and specific impulses up to 3,000 seconds is useful for near term mission planning. System power levels from $10 \mathrm{~kW}$ to $30 \mathrm{~kW}$ in steps of $2.5 \mathrm{~kW}$ are examined, while the specific impulse ranges from 1,000 seconds to 3,000 seconds in steps of 500 seconds and the initial mass ranges from $500 \mathrm{~kg}$ to $10,000 \mathrm{~kg}$ by steps of $500 \mathrm{~kg}$. Figure 3.13 shows the range of payload masses that can be delivered within the near term power levels and specific impulse range investigated. With a payload mass in mind, it is easy to see if the range of initial masses are acceptable for a given launch vehicle. As an example, a payload mass of $2,500 \mathrm{~kg}$ can be delivered to lunar orbit by a range of initial masses, depending on the power level and specific impulse used. Most of this range, about 3,300 kg to 4,200 kg, could be launched on a Delta IV Medium, which can launch up to $3960 \mathrm{~kg}$ into a $185 \mathrm{~km} \times 35,786 \mathrm{~km}, 28.5^{\circ}$ inclination orbit[18], provided that a suitable power and specific impulse is chosen.

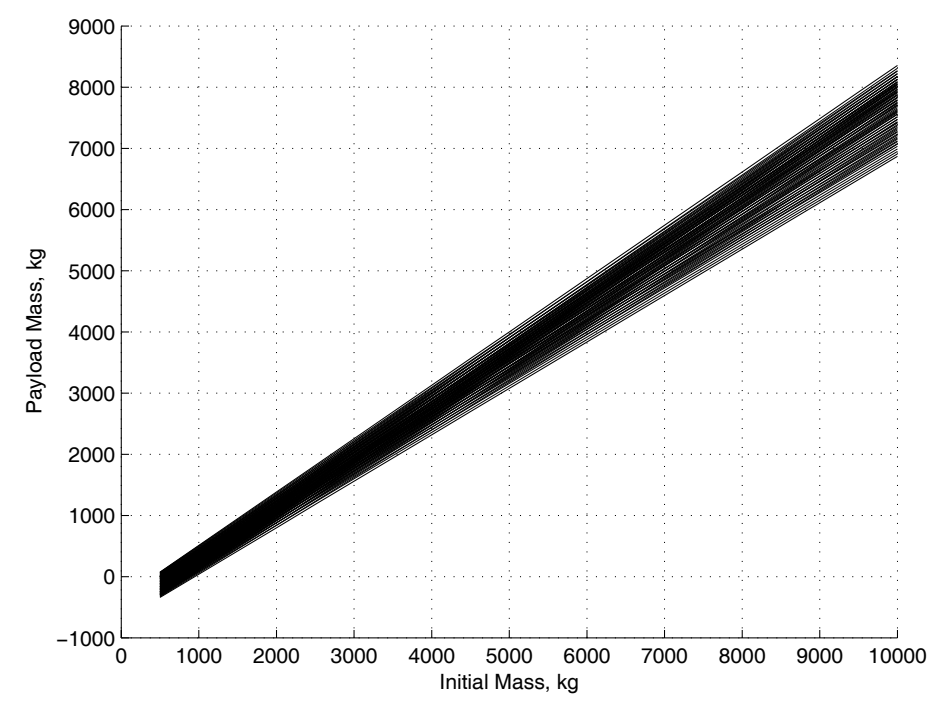

Figure 3.13: Payload Masses for all Specific Impulses and Power Levels, Near Term

Again, it is useful to examine the required initial mass, for each power level and specific impulse, minimally bounded by a positive payload mass requirement and 
a maximum boundary limited by a one year transfer time to lunar orbit. This is particularly helpful in determining what combinations of power and specific impulse will allow successful completion of the mission given a launch vehicle mass constraint.

Figure 3.14 shows the overall requirements on initial mass. For the most part, a minimum initial mass of $1000 \mathrm{~kg}$ is required to achieve a positive payload in LLO. Using a $10 \mathrm{~kW}$ power level and any of the specific impulses, payload can be delivered using a $500 \mathrm{~kg}$ initial mass; however, these payload masses are very small with the largest around $73 \mathrm{~kg}$, or a payload mass ratio of only $14.5 \%$. At a power level of $12.5 \mathrm{~kW}$ or higher, an initial mass of $1000 \mathrm{~kg}$ allows a much larger payload to be delivered, around $500 \mathrm{~kg}$, or a payload mass ratio of about $50 \%$. Considering that a small launch vehicle, like a Delta II, can launch somewhere around $2100 \mathrm{~kg}$, it is advantageous to use a higher power level. The specific impulse has a minimal impact on the minimum initial mass. The maximum initial mass, the top band, is more widely spread due to the fact that this boundary is dependent upon the maximum transfer time, which is dependent on the specific impulse. The greater the specific impulse, the longer the transfer, which reduces the amount of mass that can be transferred in a given period of time.

Examining the upper right corner, it can be seen that there is no restriction on initial mass, at least within the investigated range. This says that for all the power and specific impulses investigated, any initial mass can be transferred in under one year. And as the specific impulse decreases the restriction on the power levels lessens to allow lower powers for the same initial mass. As an example, an Atlas V 541 can lift about $8255 \mathrm{~kg}$ into GTO[19]. Clearly, there are only certain combinations of specific impulse and power to achieve this goal. If we choose a $20 \mathrm{~kW}$ power system we could use an specific impulse lower than 2250 seconds. Once the launch vehicle is chosen, the main drivers in the choice are payload mass, transfer time, and cost.

Since the near term levels are the most interesting foreseeable application, we can examine how the payload mass is directly restricted. Figure 3.15 presents the payload masses, achievable in one year or less, for a given specific impulse and power level. It is quite clear that near term technology can deliver several metric tons of payload to LLO, far more capability than a Delta II can achieve. 


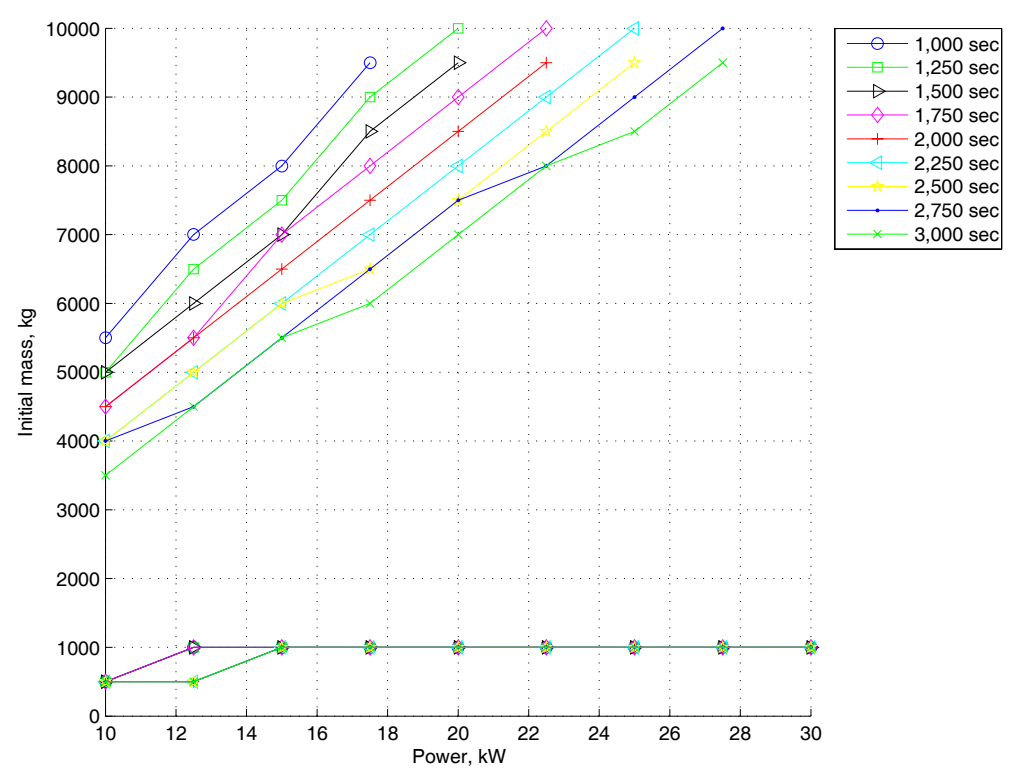

Figure 3.14: Constrained Initial Mass as a Function of Power

To clearly see the effect of specific impulse on the payload mass, we can look at Figure 3.16. This plot shows the maximum payload mass that can be achieved for each specific impulse using any combination of initial mass and power level in the examined range. Clearly, as the specific impulse increases, less propellant is used resulting in a larger payload mass in lunar orbit. Figure 3.17 is also helpful; we can see how the initial mass and the power level affects the payload mass. The $15 \mathrm{~kW}$ power level outperforms the $30 \mathrm{~kW}$ power level by payload mass alone and it is obvious that by increasing the initial mass, the payload mass will follow. Figure 3.18 is a plot of the power level versus payload mass; as the power level increases, the payload mass for a given initial mass and specific impulse decreases. This occurs since the powerplant mass increases as the power level does. You can also see that increasing the specific impulse affects the higher initial masses more than low initial masses; the difference in payload mass due to tripling the specific impulse is proportional to the increase of the initial mass. The difference in payload mass due to specific impulse is about $450 \mathrm{~kg}$ for the $4000 \mathrm{~kg}$ initial mass and about $900 \mathrm{~kg}$ for the $8000 \mathrm{~kg}$ initial mass. 


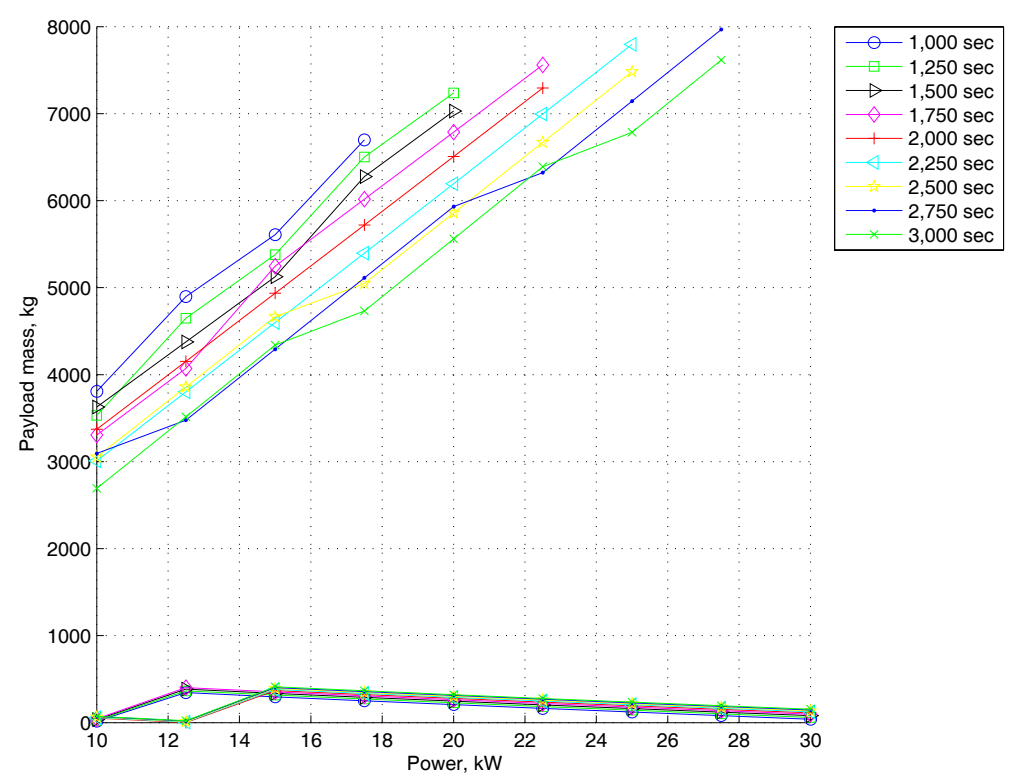

Figure 3.15: Constrained Payload Mass

Now turning attention to the transfer time for this example, we expect that it will be greater than that of the RPC, the question is whether the difference is small enough to be acceptable. Remember, at this point we have already constrained the problem such that all the transfer times are under one year. Figure 3.24 highlights the transfer time as a function of initial mass for the Delta II example. For the Delta II case, the transfer time turns out to be about 148.5 days, or not quite five months.

Solely looking at the payload mass does not take the transfer time into account. This is an important variable, especially if this system needs to compete with chemical propulsion systems and cost may directly correlate, depending on the level of autonomy. Examining only transfer time is also limiting. Looking at the payload mass delivered per day is perhaps more helpful in finding an optimum system, given requirements for a future mission, most prominently a transfer time under one year. The payload mass per day is shown in Figure 3.20. Through these plots it is evident that the payload per day ratio does not reach a maximum in the range of initial masses investigated. However, at some point there may be a region of diminishing returns, which is discussed in the next section. 


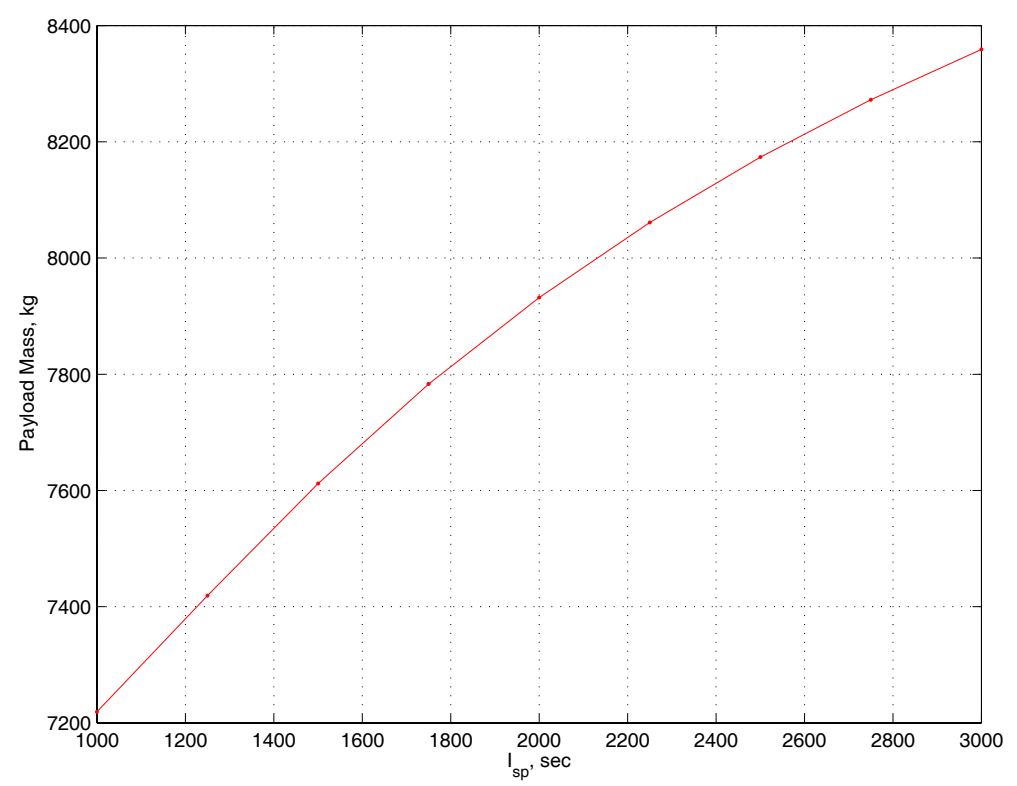

Figure 3.16: Maximum payload mass for each specific impulse

\subsubsection{Diminishing Returns - Near Term}

If we use the Delta II as the launch vehicle for near term RLEP missions, constraining the initial mass to a maximum of $2100 \mathrm{~kg}$ and the power level to a maximum of $20 \mathrm{~kW}$, we find that there is no point where increasing the initial mass gives diminishing returns. In other words, there is no optimum initial mass but to use the maximum of $2100 \mathrm{~kg}$; the corresponding power level is the maximum of $20 \mathrm{~kW}$, which is evident by examining Figure 3.20. Also, the specific impulse chosen to maximize the payload mass delivered per day should be the lowest, which is 1500 seconds for this investigation. By using a low specific impulse, the payload mass delivered per day is increased since that also means a high thrust level and therefore lower transfer time. Such is not the case at power levels and initial masses in the mid and far terms as will be shown; however, for the near term, these quantities are severely constrained. There is still more to gain by increasing the power levels and initial masses in the mid and far term ranges.

Again, we look at what point adding initial mass will bring diminished returns. 


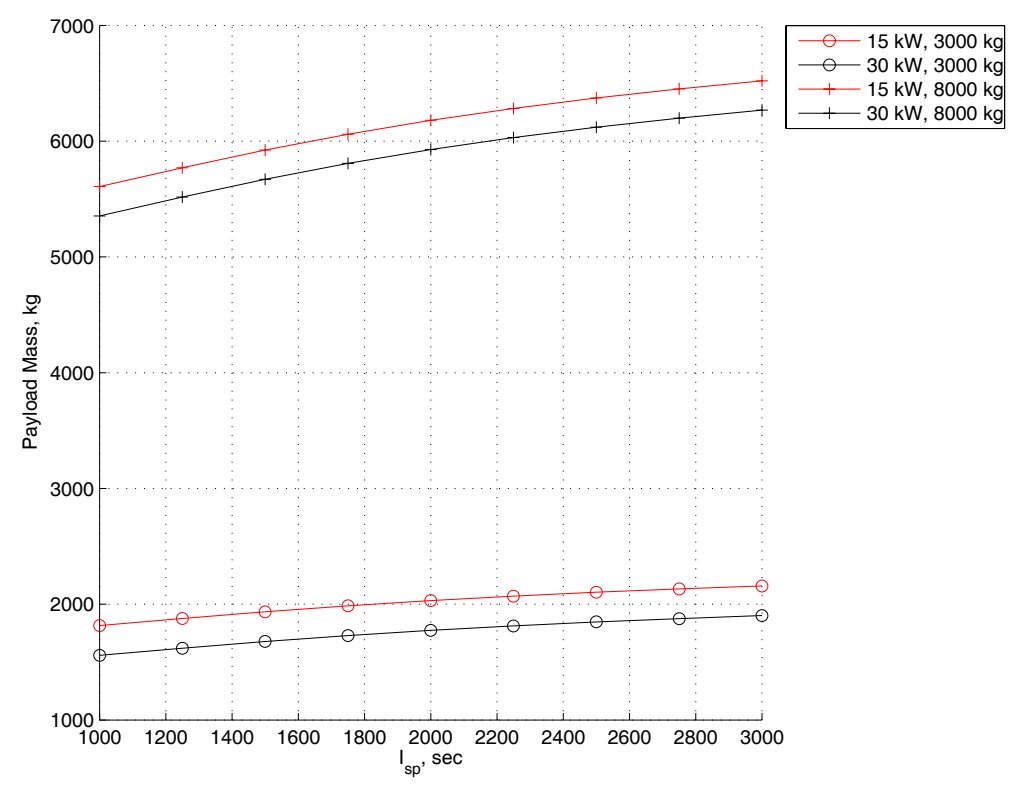

Figure 3.17: Specific impulse versus payload mass

Figure 3.21 shows the points of diminishing returns as a function of specific impulse. As previously stated, the result decreases with increasing specific impulse since it is inversely proportional to thrust. A couple of the points on this plot do not follow the general trend as closely as might be expected. This is basically due to the fact that the trade space, the investigated ranges of inputs, is constrained. Within this trade space, some of the combinations of inputs do not give a point of diminishing returns.

Figure 3.22 shows the transfer times for the points of maximum optimum payload mass delivered per day, the points of diminishing returns, as a function of specific impulse. Quickly we can see that all these cases fall within the one year limit. And Figure 3.23 shows the points of diminishing returns by power level.

Figure 3.24 highlights the transfer times for all the specific impulses, from 1000 seconds to 3000 seconds. For any specific impulse in the examined range, a Delta II payload will reach lunar orbit in under one year. 


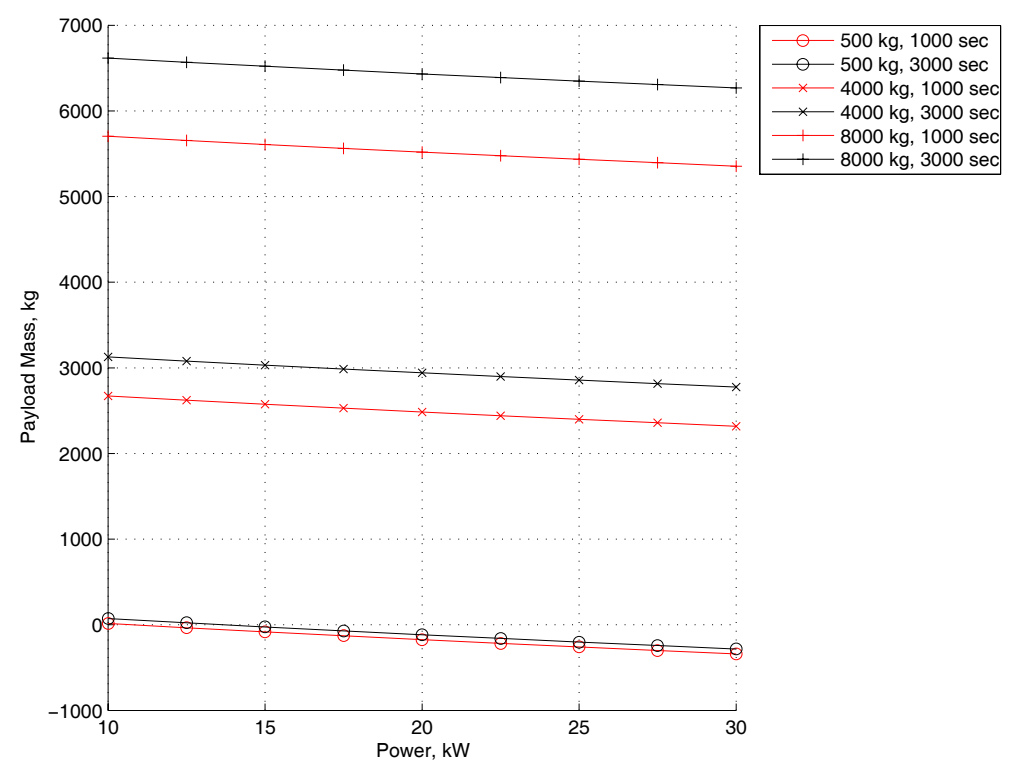

Figure 3.18: Power versus payload mass

\subsubsection{Possible Payloads}

At this point we can examine what sort of payload mass this system will allow. Continuing with the Delta II near term example, we can examine closely the payload mass in Figure 3.25; clearly using the highest power level does not result in the largest payload mass but it does result in the largest payload mass delivered per day. Using a $10 \mathrm{~kW}$ power level, the payload mass is about $1230 \mathrm{~kg}$ which is a payload mass fraction of about $58.6 \%$. For comparison the conventional chemical baseline RPC has a payload mass fraction of about $12 \%$, approximately $250 \mathrm{~kg}$.

Clearly, there is a large advantage in using an ERPS. This mass margin can be used in numerous ways; extra science instruments, deep drilling tools, technology demonstrations (enough mass for backups using conventional equipment), or lunar rovers could be employed. Unmanned or manned rovers of a range of sizes[20] allow for a large area of exploration, up to ranges of tens of kilometers. For example, a Boeing design for a manned light utility rover has a mass of $984 \mathrm{~kg}[21]$, meaning this system could also transport small amounts of cargo for manned support missions. 


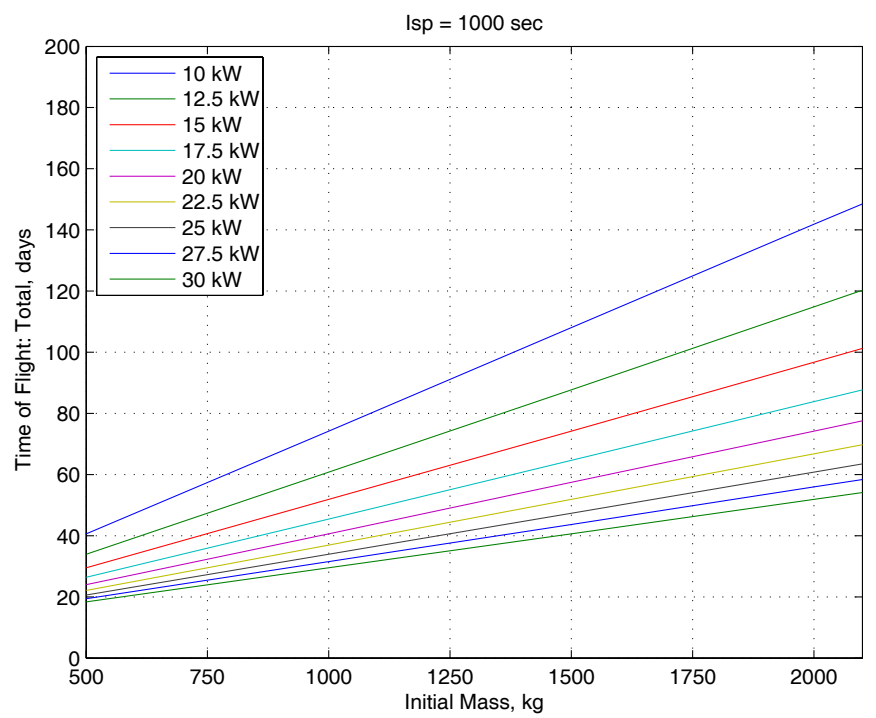

Figure 3.19: Initial Mass vs. Transfer Time, Delta II example
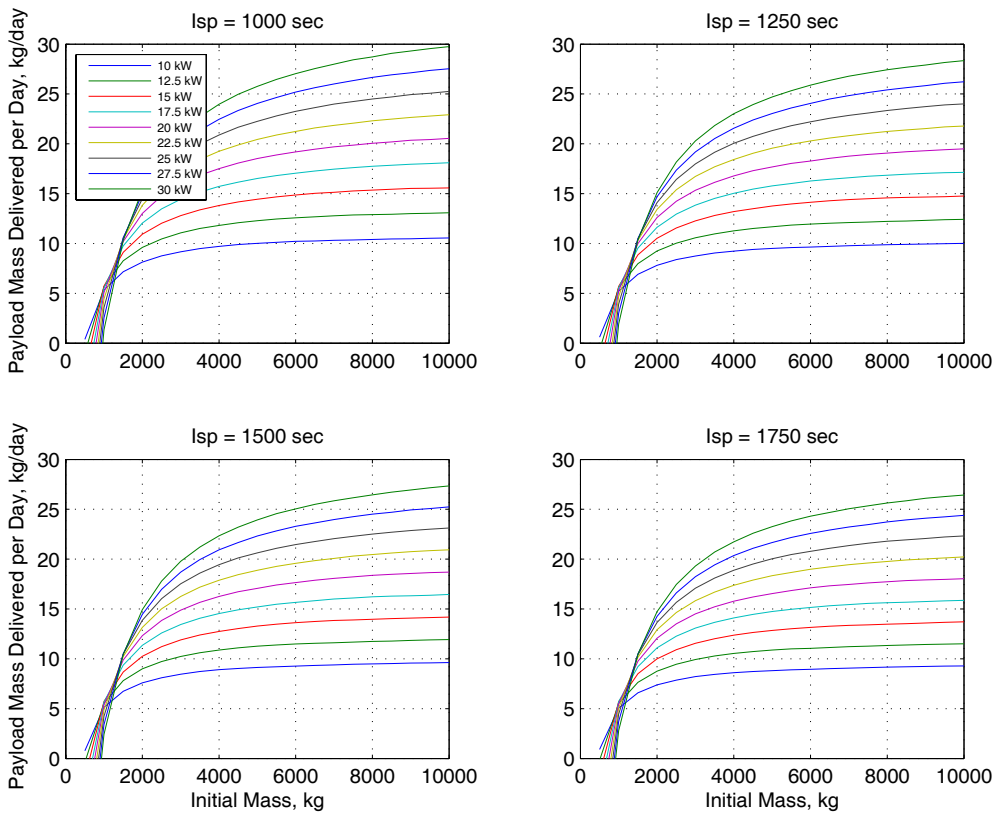

Figure 3.20: Payload Mass per Day as a Function of Initial Mass 


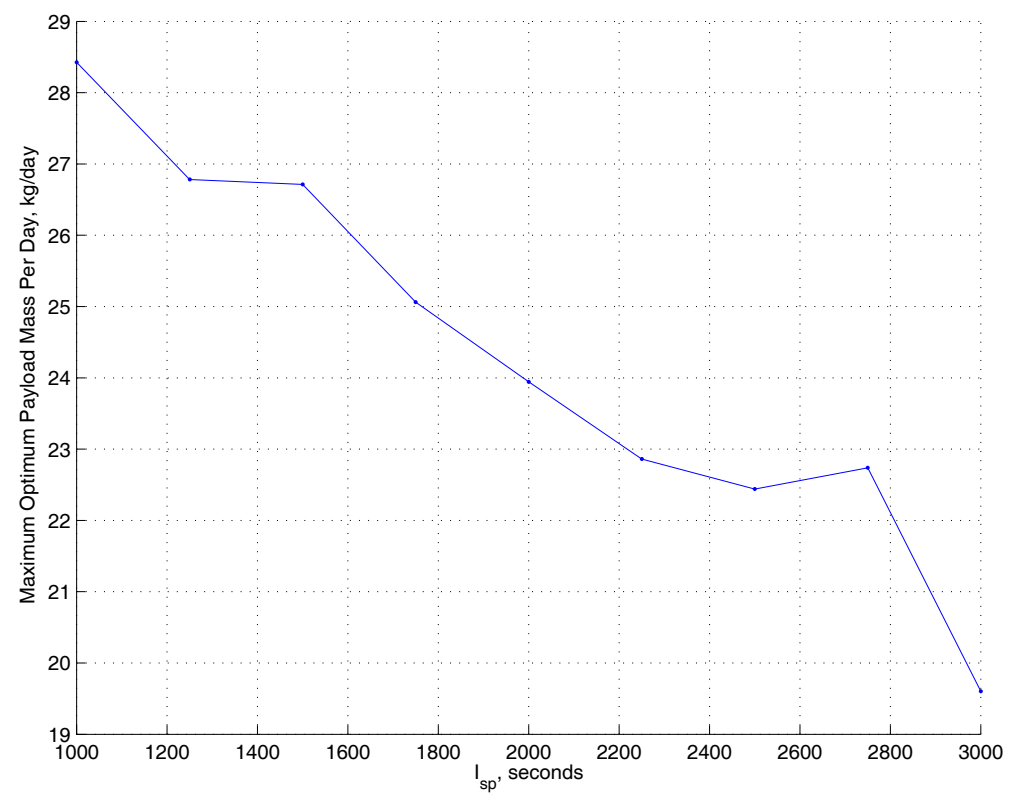

Figure 3.21: Initial Mass Diminishing Returns as a Function of Specific Impulse

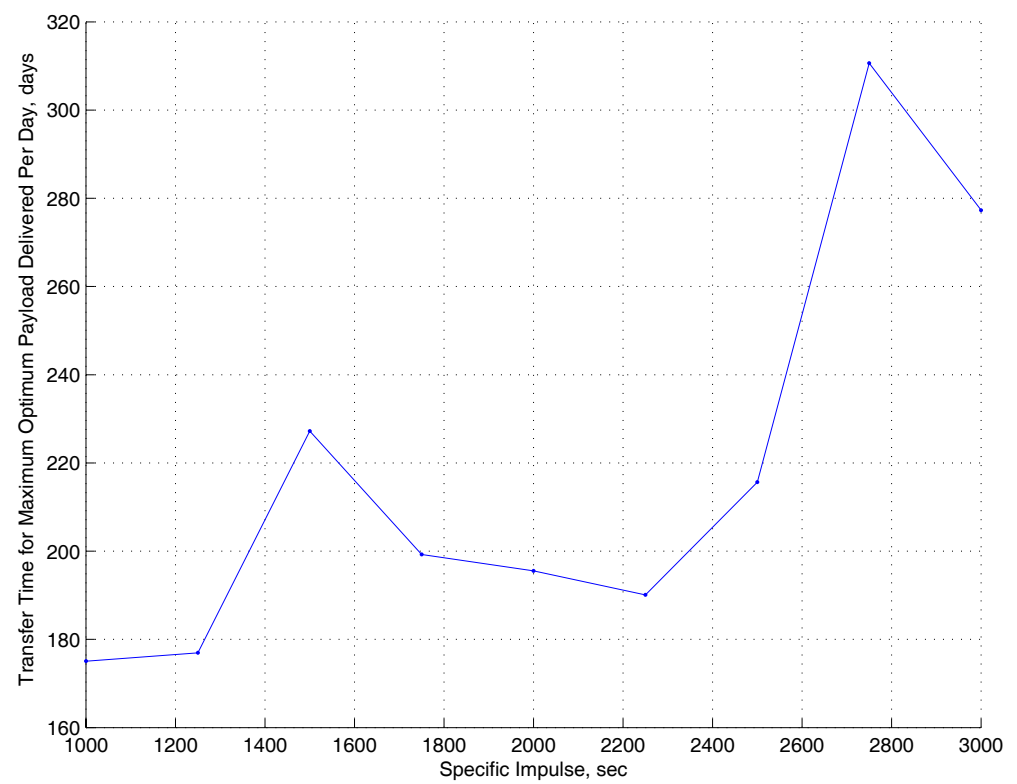

Figure 3.22: Transfer Time as a Function of Specific Impulse for the Maximum Optimum 


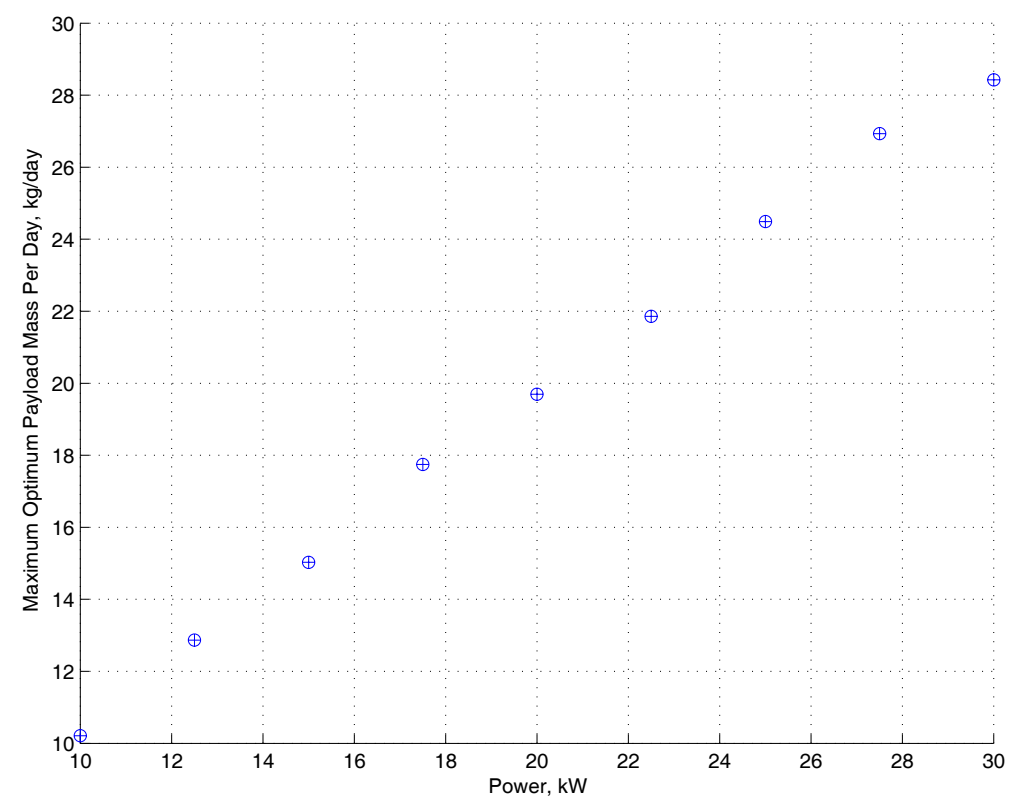

Figure 3.23: Initial Mass Diminishing Returns as a Function of Power

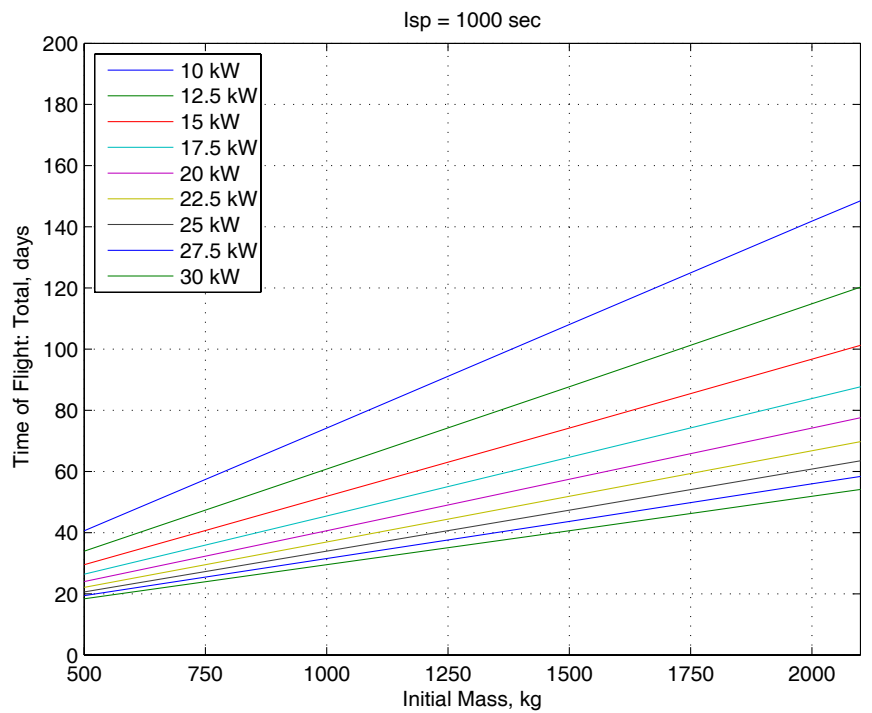

Figure 3.24: Initial Mass vs. Transfer Time, Delta II example 


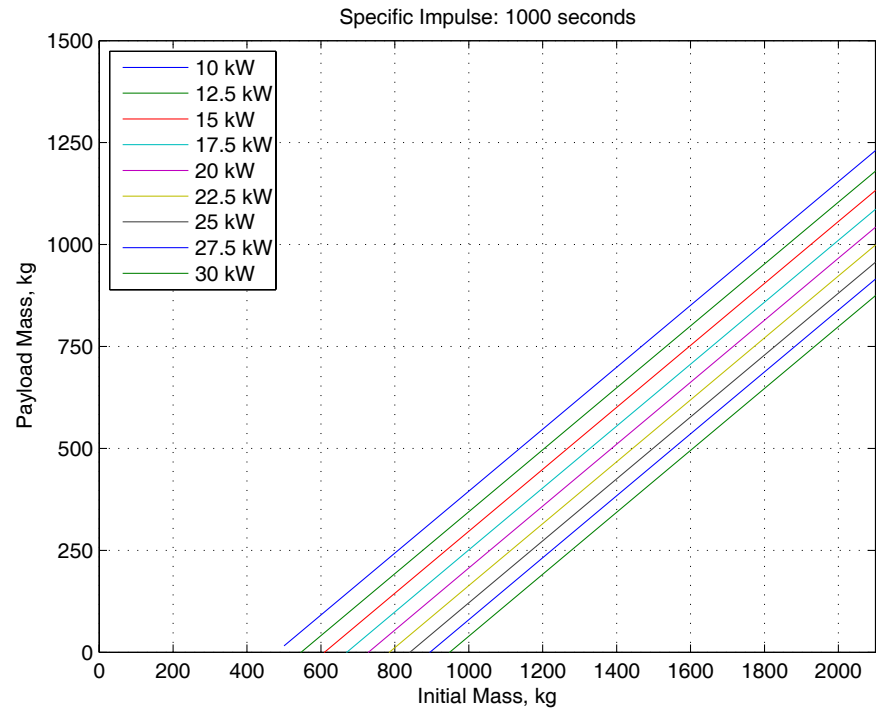

Figure 3.25: Initial Mass vs. Payload Mass per Day, Delta II example 


\subsection{Mid Term}

This section will highlight the mid term results, where the power ranges from $30 \mathrm{~kW}$ to $100 \mathrm{~kW}$ and the specific impulse ranges from 3,000 seconds to 15,000 seconds. Again, we will first examine the required initial mass, for each power level and specific impulse, Figure 3.26. The maximum initial mass lines end at 10,000 kg initial mass, where the investigated range ends. Again, the region between the two sets of lines indicates a combination of initial mass, specific impulse, and power level that will result in a delivery of a positive payload mass in under one year to lunar orbit. As an example, a Delta II sized payload could be launched using any of the combinations of power and specific impulse in this investigated range. For an Atlas V 541 example, a large range of specific impulse is able to be used with the minimum around 4500 seconds and a power level minimum around $35 \mathrm{~kW}$.

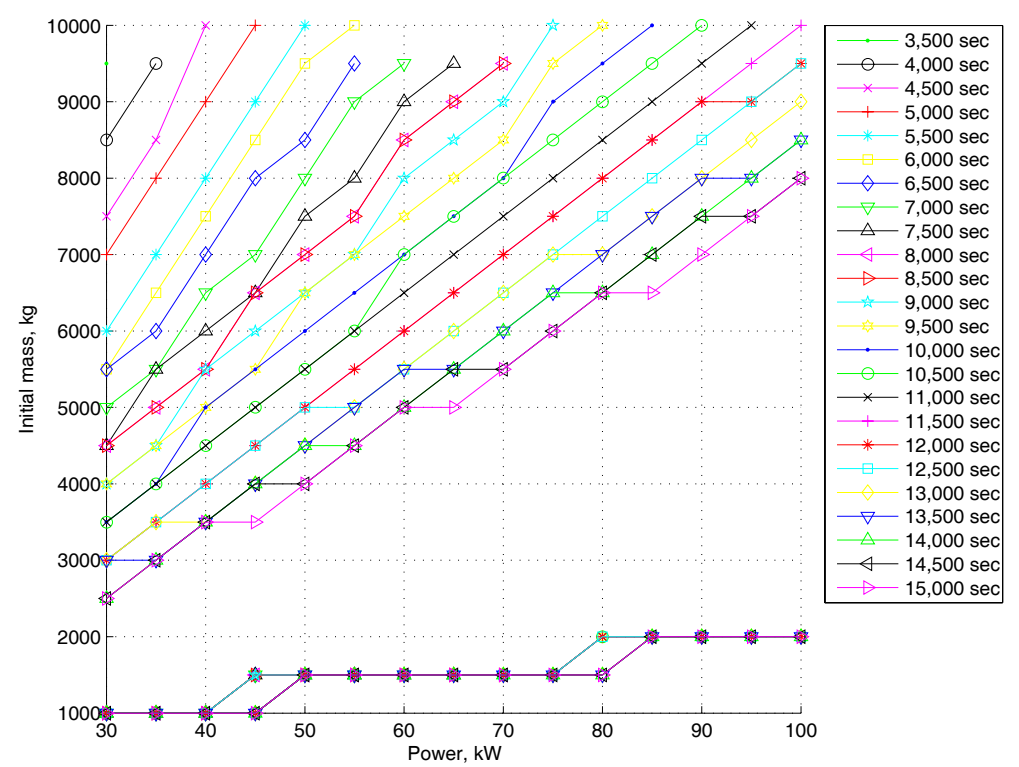

Figure 3.26: Constrained Initial Mass

Now we move to an investigation of the relationship between power and trip time. Figure 3.27 shows power versus the maximum and minimum total transfer time (within the range of initial masses) for the specific impulse range specified above. Each 
line has constant specific impulse and is either the maximum or minimum transfer time for all the initial masses. The lower grouping consists of all the minimum transfer times and the upper grouping of all the maximum transfer times for the specific impulse range. Since a large portion result in long transfer times, there may be a restriction on specific impulse. Most obviously, the transfer time drops as the power increases; this result is expected as can be seen through Equation 1.3. As power increases so does thrust and therefore decreases the transfer time.

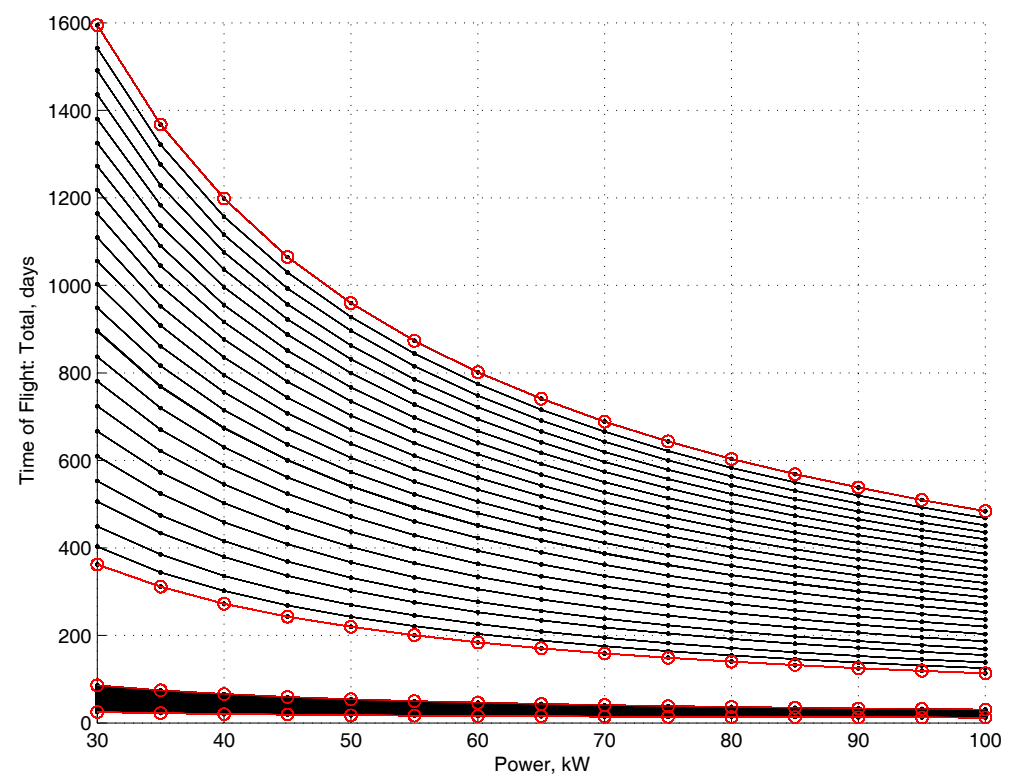

Figure 3.27: Power vs. Total Time of Flight for a Range of Specific Impulse, Midterm

Figure 3.28 is indicative of the transfer time as a function of the power level. The top line is the maximum transfer time, and the bottom line the minimum, for the given specific impulse as a function of power for all the initial masses in the investigated range. This plot shows only one specific impulse as a representative. Figure 3.29 presents the initial mass versus the total transfer time split to show each specific impulse separately. This plot shows only one specific impulse as a representative.

Plotting the payload mass transported per day versus the initial mass, Figure 3.30 shows that as specific impulse increases the amount of payload mass delivered per day decreases. There are fifteen power levels shown in these plots, from $30 \mathrm{~kW}$ to 

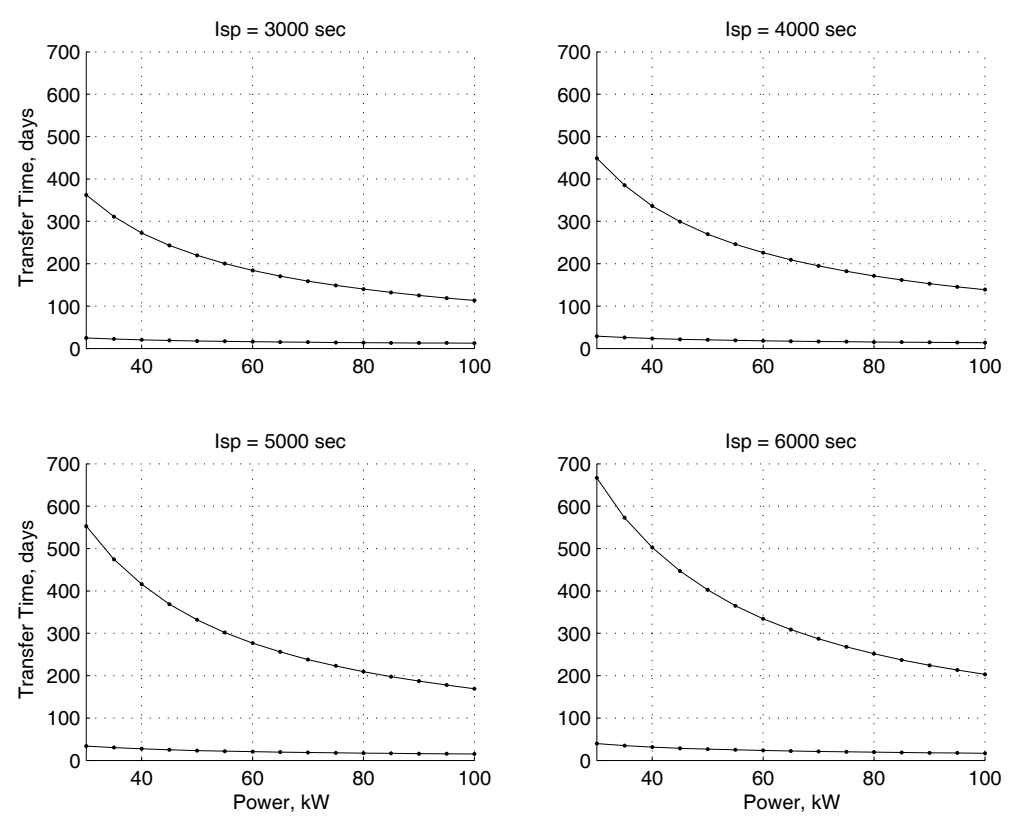

Figure 3.28: Total Time of Flight as a Function of Power

$100 \mathrm{~kW}$ (increments of $5 \mathrm{~kW}$ ) and the bottom curve, the blue curve, corresponds to the lowest power level which increases vertically. As the power increases so does the amount of mass delivered per day. The variation in specific impulse plays a strong role; by increasing this quantity from 3000 seconds to 15,000 seconds the payload mass delivered to lunar orbit per day drops by about $45.5 \mathrm{~kg}$. System power has a large effect as well, a variation of $62 \mathrm{~kg} /$ day to $22 \mathrm{~kg} /$ day results at an initial mass of $10,000 \mathrm{~kg}$ and specific impulse of 3000 seconds.

To more clearly show the importance of specific impulse, Figure 3.31 presents the maximum payload mass that can be achieved for each specific impulse for the investigated range of power levels and initial masses. Also, Figure 3.32 presents some selected results of the effect of specific impulse on the transfer time. This figure shows the specific impulse from 3,000 seconds to 15,000 seconds; the effect is clearly seen as the transfer time increases by hundreds of days over the range. 

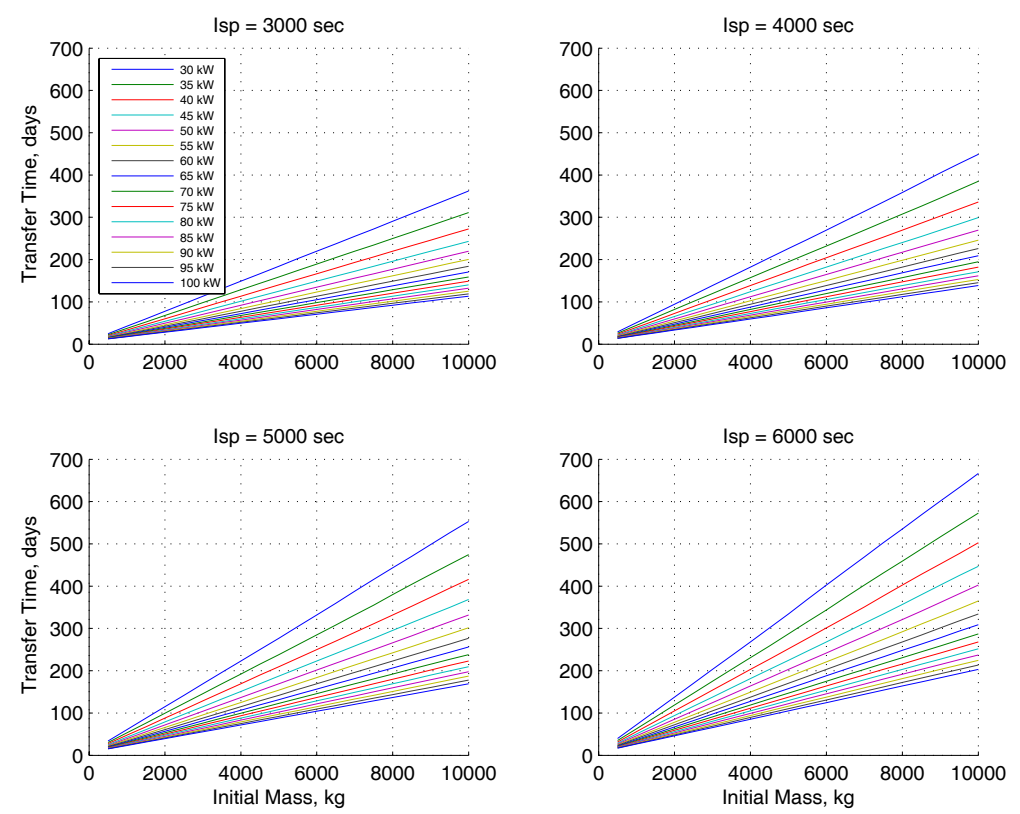

Figure 3.29: Initial Mass vs. Total Time of Flight, Midterm

\subsubsection{Diminishing Returns - Mid Term}

Figure 3.33 shows the points of diminishing returns as a function of specific impulse. As previously stated, the result decreases with increasing specific impulse since it is inversely proportional to thrust.

Figure 3.34 shows the transfer times for the points of maximum optimum payload mass delivered per day, the points of diminishing returns, as a function of specific impulse. Quickly we can eliminate those high specific impulses where the transfer time is above one year. This eliminates the 10,500 seconds, 11,500 seconds, 12,500 seconds, 13,000 seconds, 13,500 seconds, 14,000 seconds, 14,500 seconds, and 15,000 seconds specific impulses. This leaves specific impulses of 3000 seconds to 10,000 seconds, 11,000 seconds and 12,000 seconds unless there is some reason to operate at nonoptimum conditions.

Figure 3.35 shows the points of diminishing returns by power level. A couple of the points on this plot do not follow the general trend as closely as might be expected. This is especially true for the $85 \mathrm{~kW}$ case. This is basically due to the fact that 

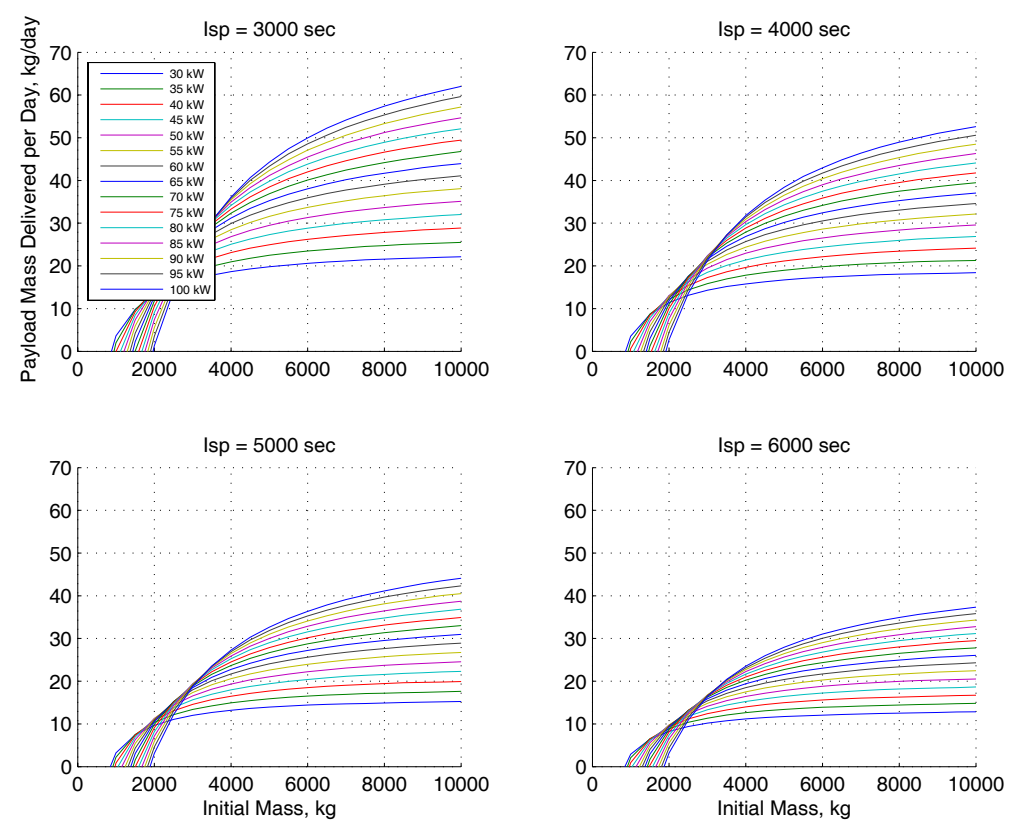

Figure 3.30: Initial Mass vs. Payload Mass per Day, Midterm

the trade space, the investigated ranges of inputs, is constrained. Within this trade space, some of the combinations of inputs do not give a point of diminishing returns. At power levels of $80 \mathrm{~kW}$ and $90 \mathrm{~kW}$, the maximum result come at a low specific impulse. Comparing this with the $85 \mathrm{~kW}$ data, we can see that there is no point of diminishing returns at low specific impulses. This curve of initial mass versus payload mass delivered per day is close to reaching that point. It could be expected that by increasing the range of initial masses to $15,000 \mathrm{~kg}$ would reveal a point of diminishing returns.

\subsubsection{Possible Payloads}

At this point we can examine what sort of payload mass this mid term system will allow. Within the mid term ranges of power and specific impulse we can attempt to find the best combination. Plotting the optimum payload mass as a function of specific impulse we can see that they lie within a band of about $2000 \mathrm{~kg}$, Figure 3.36. From previous results, Figure 3.33, we would be tempted to choose a low specific 


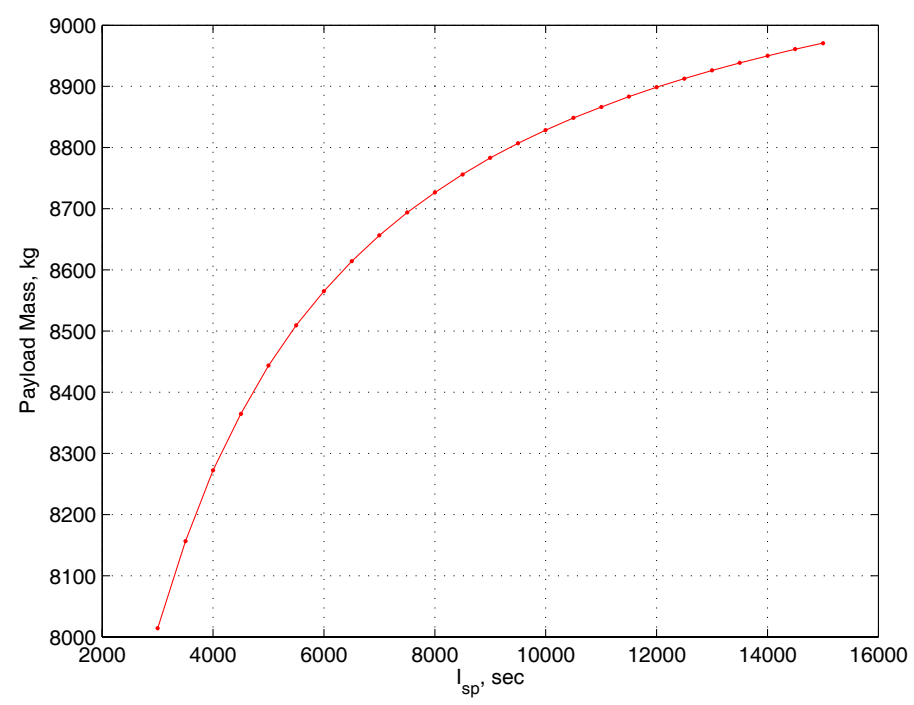

Figure 3.31: Maximum Payload Mass for Each Specific Impulse, Midterm

impulse to achieve a high efficiency with respect to both payload mass delivered and transfer time; a choice of this nature leads to about a $6000 \mathrm{~kg}$ payload mass. However, if transfer time could increase from around 200 days, nearer to the limit of one year, about $7000 \mathrm{~kg}$ could be delivered.

Compared with the near term technology discussed in the previous section, there is a large increase in capability. The near term technology gave results of payloads in the vicinity of $1200 \mathrm{~kg}$ at $10 \mathrm{~kW}$. This increased capability will allow much greater payload opportunities; for example, the capability now includes landing pressurized rovers. A Boeing design called "Rover First" has a landed mass of about $4300 \mathrm{~kg}[21]$, well below the mid term technology capability. Small habitats, on the order of $7000 \mathrm{~kg}[22]$, could also be delivered to orbit. These sorts of payloads highlight the advantages of using electric propulsion. For comparison the conventional chemical baseline RPC has a payload mass fraction of about $12 \%$, approximately $250 \mathrm{~kg}$ where the payload ratio for the ERPS is about $78.75 \%$ at $7087 \mathrm{~kg}$ of payload. However, the transfer time for this example is around 304 days.

In the next sections we will further investigate these results for far term uses (up 

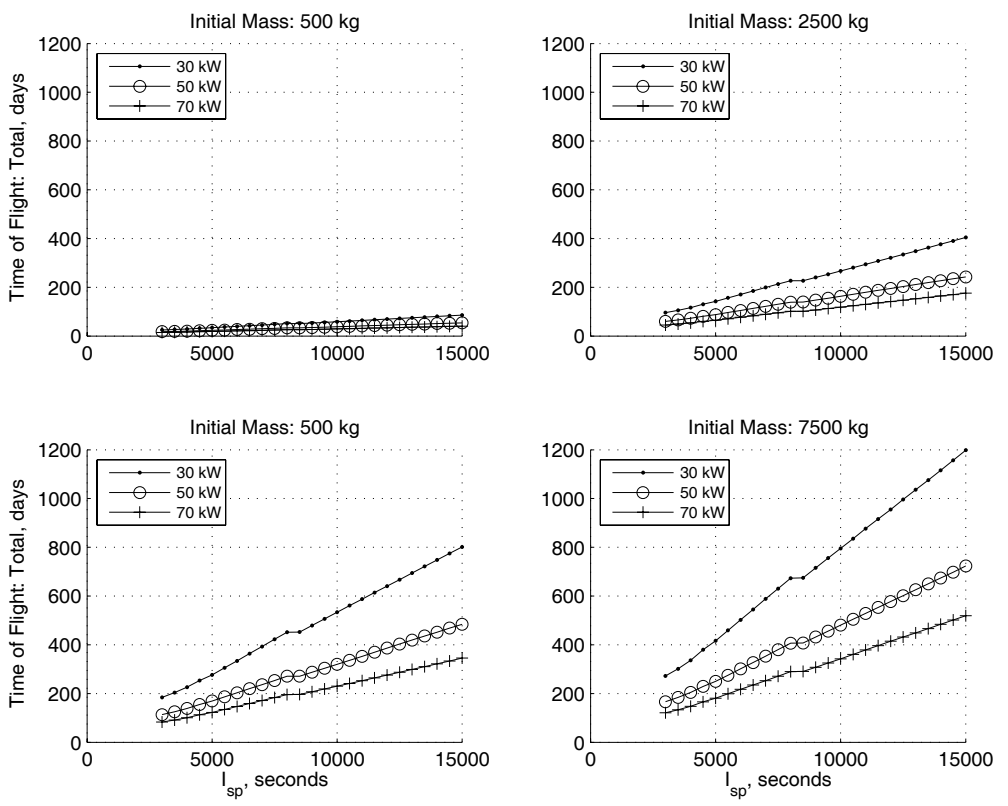

Figure 3.32: Specific Impulse vs. Transfer Time, Midterm

to $200 \mathrm{~kW}$ power and 30,000 seconds specific impulse). 


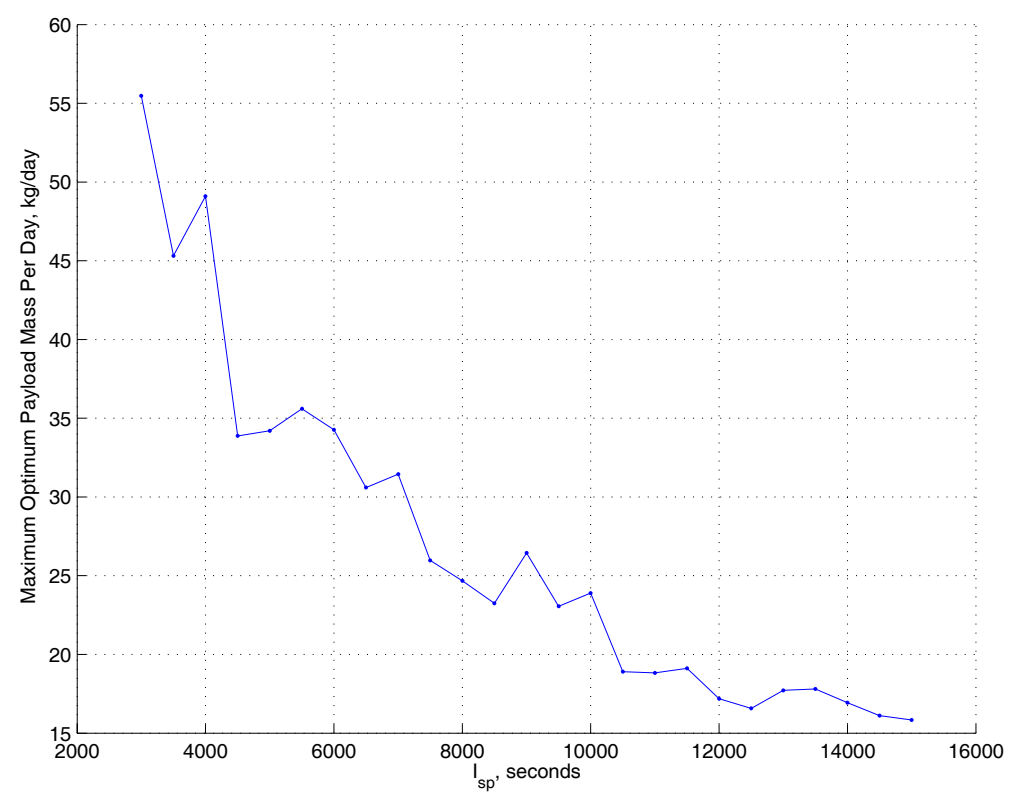

Figure 3.33: Initial Mass Diminishing Returns as a Function of Specific Impulse

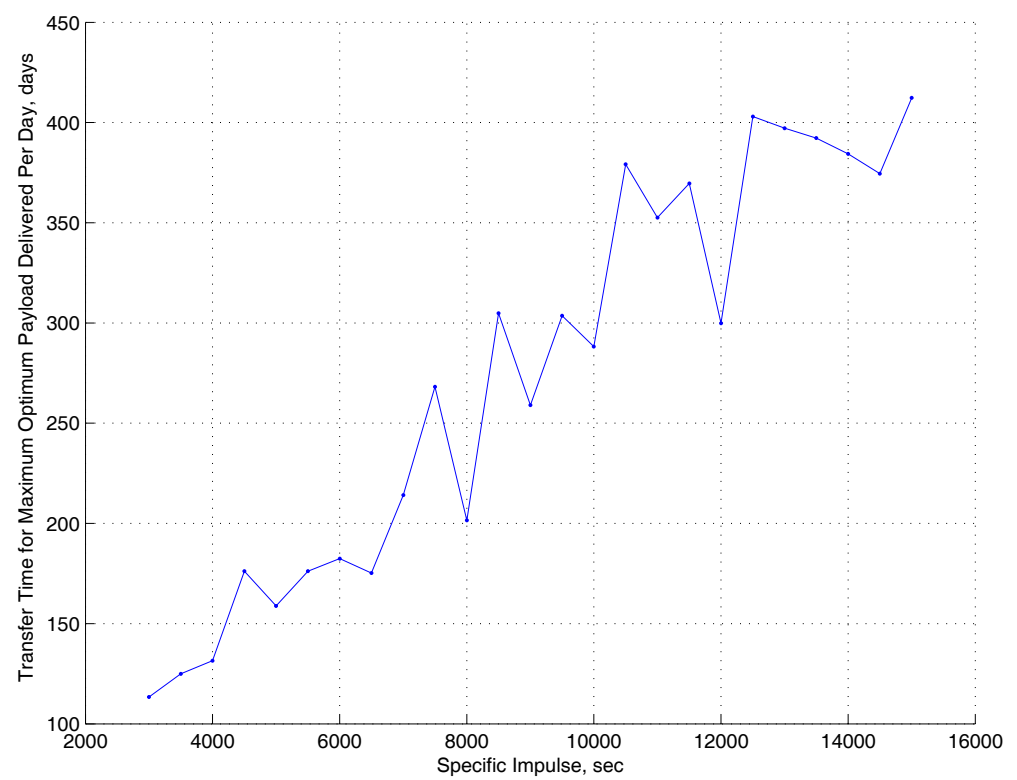

Figure 3.34: Transfer Time as a Function of Specific Impulse for the Maximum Optimum 


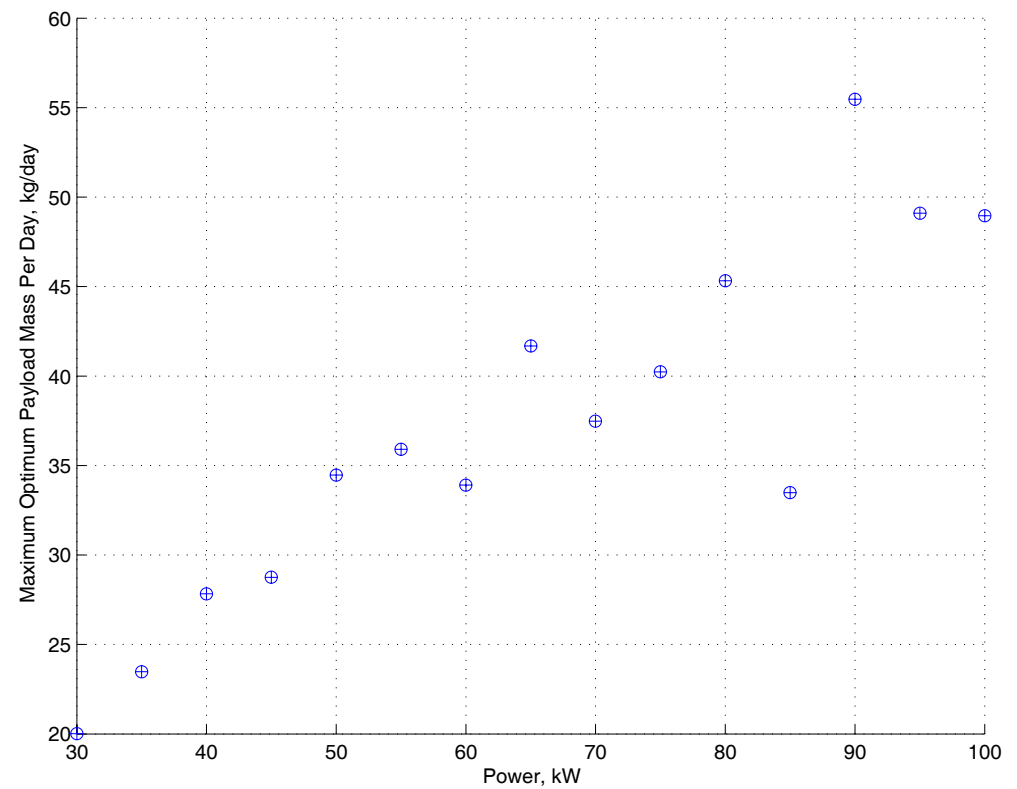

Figure 3.35: Initial Mass Diminishing Returns as a Function of Power

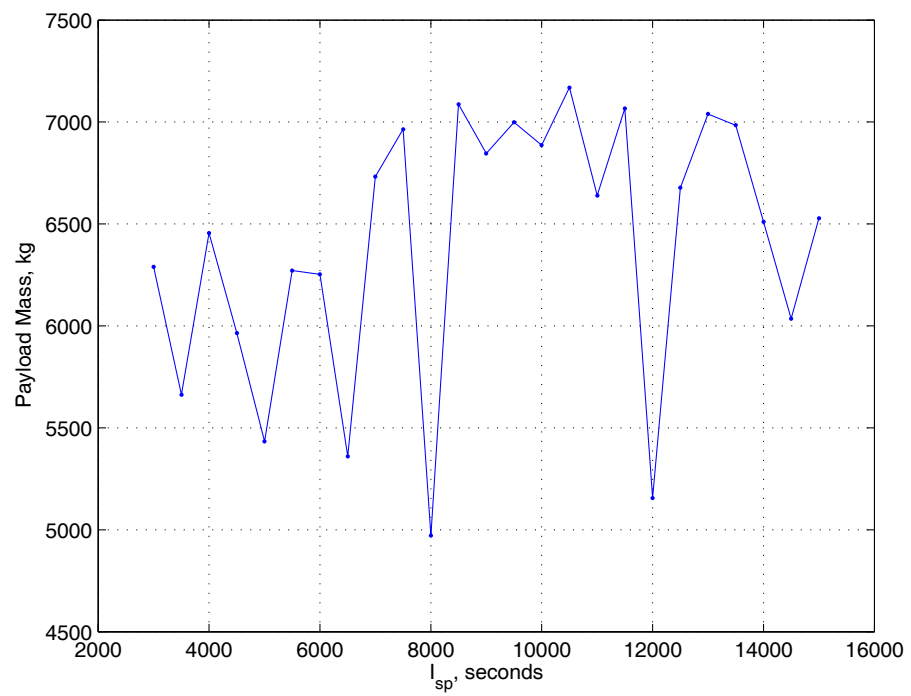

Figure 3.36: Specific impulse vs. Optimum Payload Mass 


\subsection{Far Term}

This section will highlight the far term results, where the power ranges from $100 \mathrm{~kW}$ to $200 \mathrm{~kW}$ and the specific impulse ranges from 15,000 seconds to 30,000 seconds. Again, we will first examine the required initial mass, for each power level and specific impulse, Figure 3.37. The maximum initial mass lines end at 10,000 kg initial mass, where the investigated range ends. Again, the region between the two sets of lines indicates a combination of initial mass, specific impulse, and power level that will result in a delivery of a positive payload mass in under one year to lunar orbit. Compared to the mid term levels, the requirements are more stringent for both the minimum initial mass and the maximum initial mass. This is most likely due to the assumption that the thruster efficiency, around a specific impulse of 4500 seconds, levels off. Taking a closer look at how the efficiency behaves for very high specific impulses would be interesting.

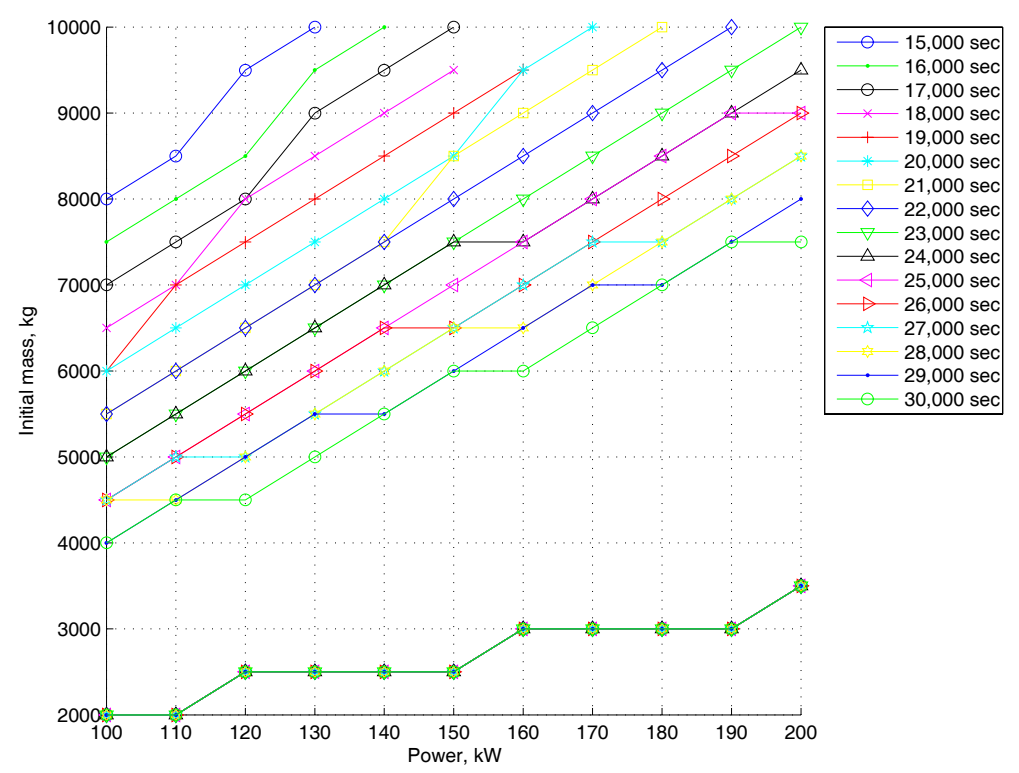

Figure 3.37: Constrained Initial Mass

Now we move to an investigation of the relationship between power and trip time. Figure 3.38 shows power versus the maximum and minimum total transfer time 
(within the range of initial masses) for the specific impulse range specified above. Each line has constant specific impulse and is either the maximum or minimum transfer time for all the initial masses. The lower grouping consists of all the minimum transfer times and the upper grouping of all the maximum transfer times for the specific impulse range. Since a large portion result in long transfer times, longer than the one year requirement, this further indicates restrictions on specific impulse.

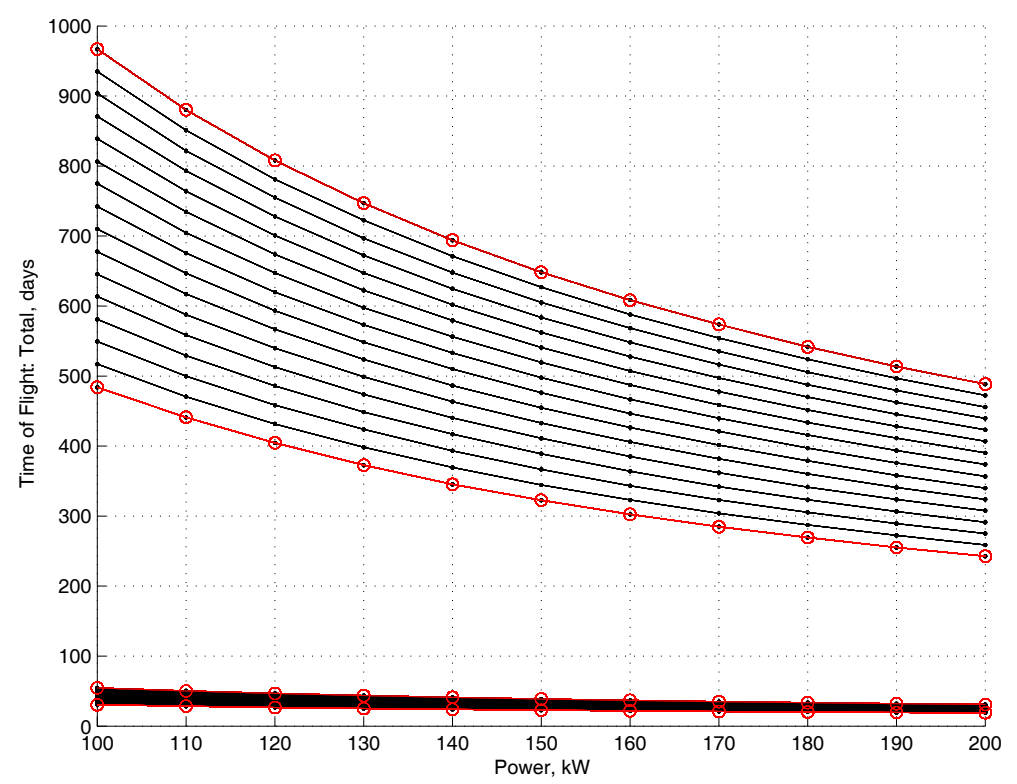

Figure 3.38: Power vs. Total Time of Flight for a range of Specific Impulse, Far Term

Figure 3.39 is indicative of the transfer time as a function of the power level. The top line is the maximum transfer time, and the bottom line the minimum, for the given specific impulse, as a function of power for all the initial masses in the investigated range. This plot shows only one specific impulse as a representative.

Figure 3.40 presents the initial mass versus the total transfer time split to show each specific impulse separately. This plot shows only one specific impulse as a representative.

We can now look at how the payload mass transported per day behaves for the inputs. Figure 3.41 shows that as specific impulse increases the amount of payload mass delivered per day decreases. There are fifteen power levels shown in these 

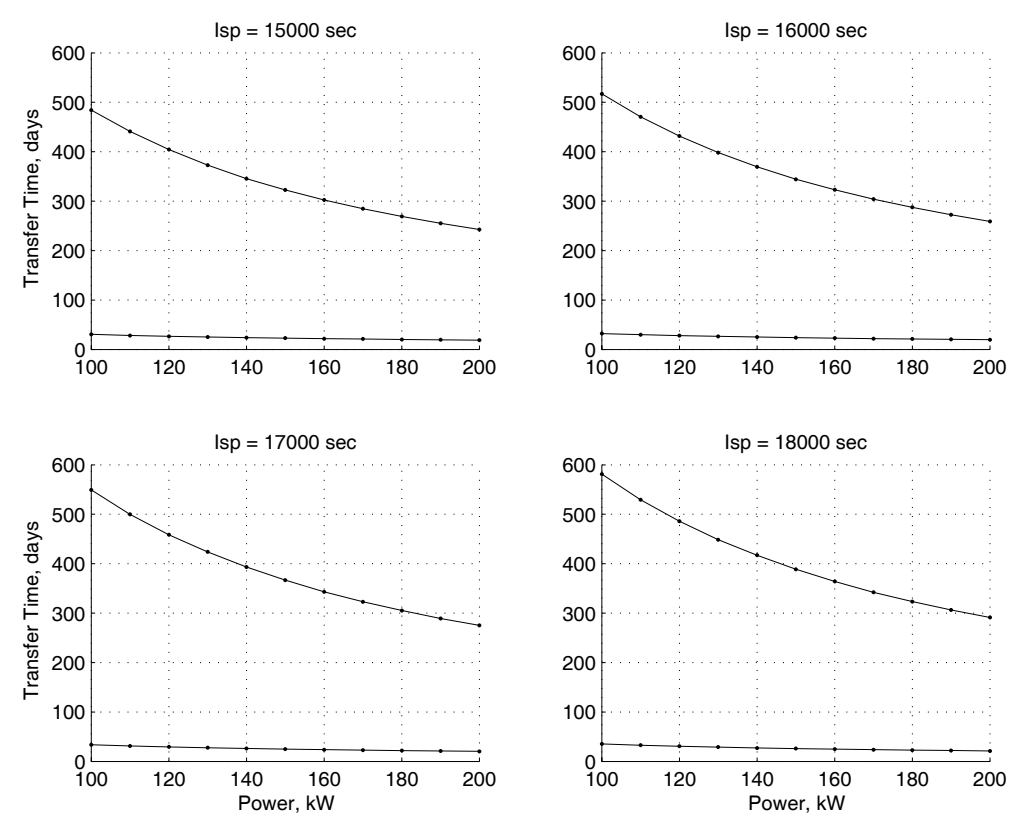

Figure 3.39: Total Time of Flight as a Function of Power

plots, from $30 \mathrm{~kW}$ to $200 \mathrm{~kW}$ (increments of $5 \mathrm{~kW}$ ) and the bottom curve, the blue curve, corresponds to the lowest power level which increases vertically. As the power increases so does the amount of mass delivered per day. The variation in specific impulse plays a strong role; by increasing this quantity from 15,000 seconds to 30,000 seconds the payload mass delivered to lunar orbit per day drops by about $13.65 \mathrm{~kg} /$ day. System power has a large effect as well, a variation of $27.75 \mathrm{~kg} /$ day at $200 \mathrm{~kW}$ to $16.5 \mathrm{~kg} /$ day at $100 \mathrm{~kW}$ exists at an initial mass of 10,000 kg and specific impulse of 15,000 seconds.

To more clearly show the importance of specific impulse, Figure 3.42 presents the maximum payload mass that can be achieved for each specific impulse within the range of investigated power levels and initial masses. Also, Figure 3.43 presents some selected results of the effect of specific impulse on the transfer time. This figure of plots shows the specific impulse from 15,000 seconds to 30,000 seconds; the effect is clearly seen as the transfer time increases by hundreds of days over this range. 

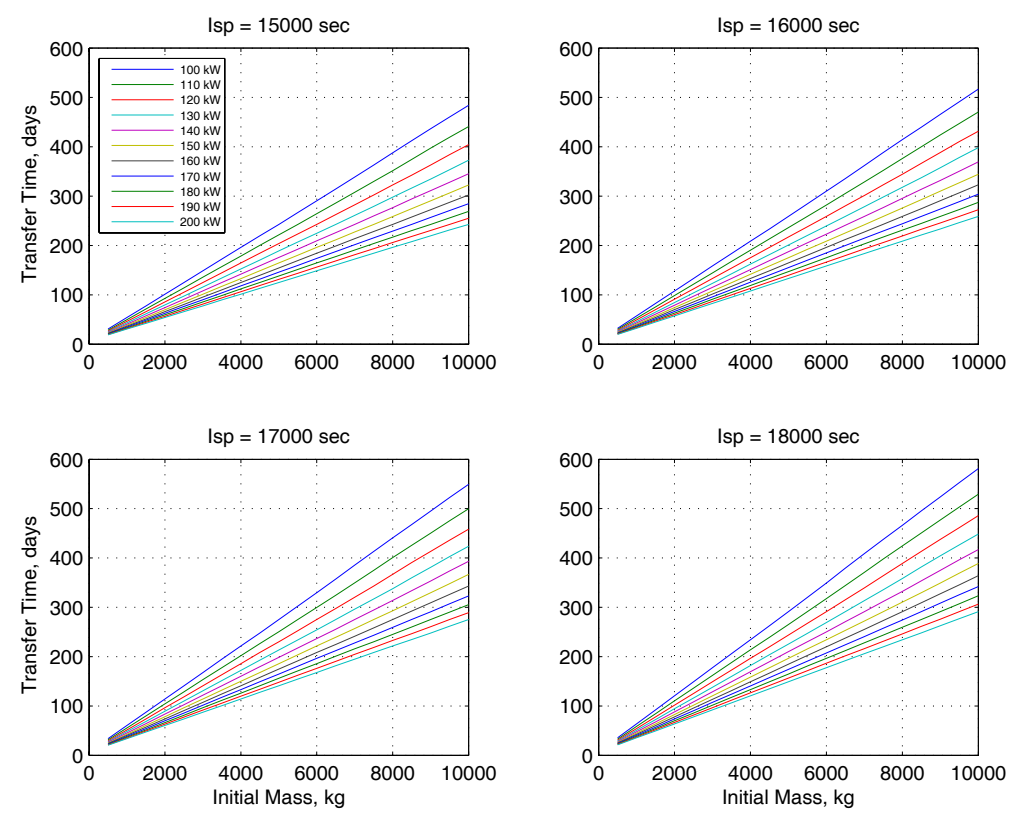

Figure 3.40: Initial Mass vs. Total Time of Flight, Far Term

\subsubsection{Diminishing Returns - Far Term}

Figure 3.44 shows the points of diminishing returns by power level. There are power levels where the result is zero occurring at $170 \mathrm{~kW}, 180 \mathrm{~kW}$, and $190 \mathrm{~kW}$. The curves of initial mass versus payload mass delivered per day at nearby power levels do not reach a point of diminishing returns.

Figure 3.45 shows the points of diminishing returns as a function of specific impulse. As previously stated, the result decreases with increasing specific impulse since it is inversely proportional to thrust. A zero point also occurs here indicating that for the specific impulse, 28,000 seconds in this case, no point of diminishing returns exists.

Figure 3.46 shows the transfer times for the points of maximum optimum payload mass delivered per day, the points of diminishing returns, as a function of specific impulse. Quickly we can eliminate those high specific impulses where the transfer time is above one year. This immediately eliminates 24,000 through 27,000, 29,000 and 30,000 seconds specific impulses. Looking closer we also see that it eliminates 

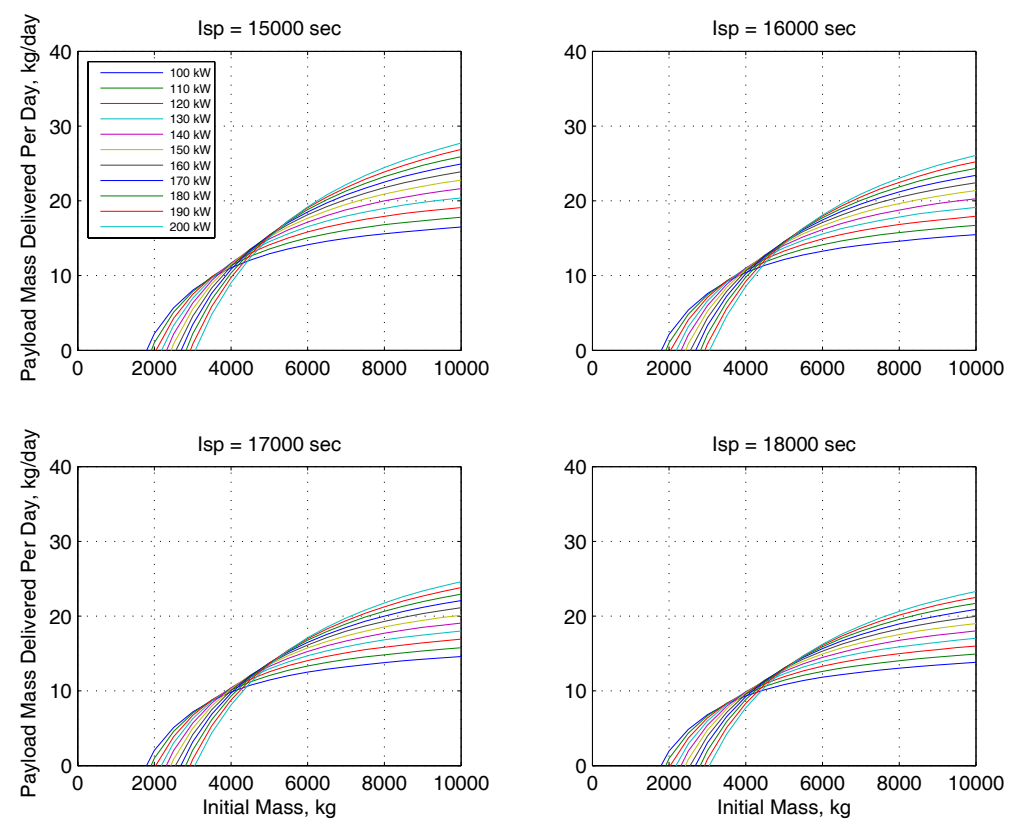

Figure 3.41: Initial Mass vs. Payload Mass per Day, Far Term

18,000 and 22,000 seconds. This leaves only 15,000 through 17,000 and 19,000 through 21,000 seconds as viable specific impulses for far term technology. I should mention again the assumption that was made at the beginning; the thruster efficiency levels off after a specific impulse of 4,500 seconds. Notice here that the 28,000 second specific impulse point is omitted.

At this point we can examine what sort of payload mass this far term system will allow. Within the far term ranges of power and specific impulse we can attempt to find the best combination. Plotting the optimum payload mass as a function of specific impulse we can see that they lie within a band of about $2000 \mathrm{~kg}$, Figure 3.47. From previous results, Figure 3.45, we would be tempted to choose a low specific impulse to achieve a high efficiency with respect to both payload mass delivered and transfer time; a choice of this nature leads to about a $6700 \mathrm{~kg}$ payload mass. 


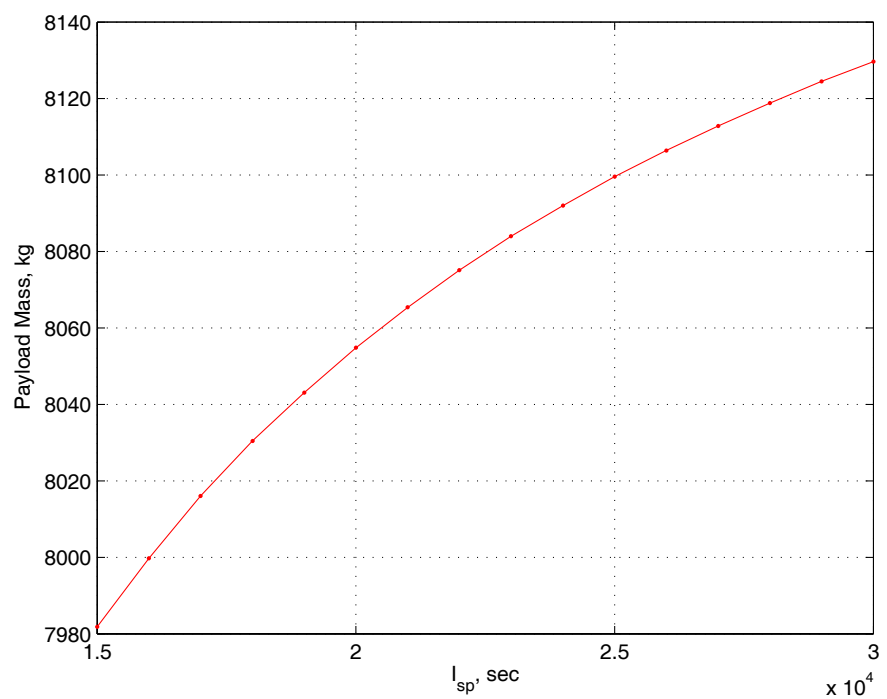

Figure 3.42: Maximum Payload Mass for Each Specific Impulse
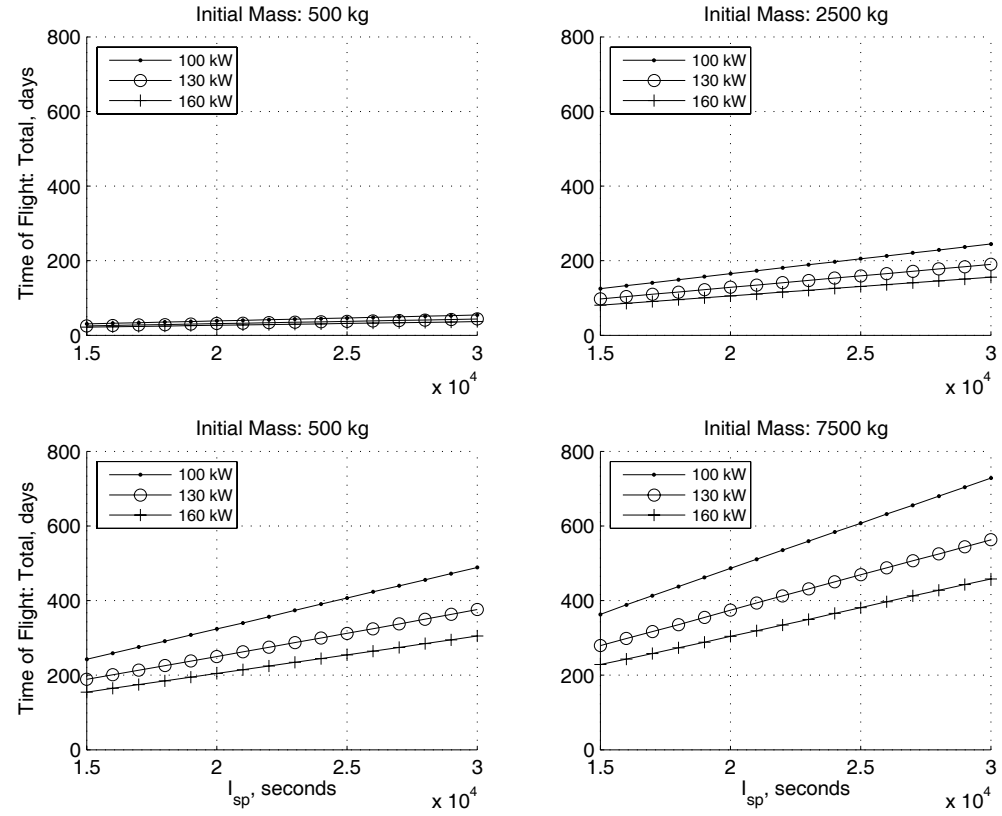

Figure 3.43: Specific Impulse vs. Transfer Time, Far Term 


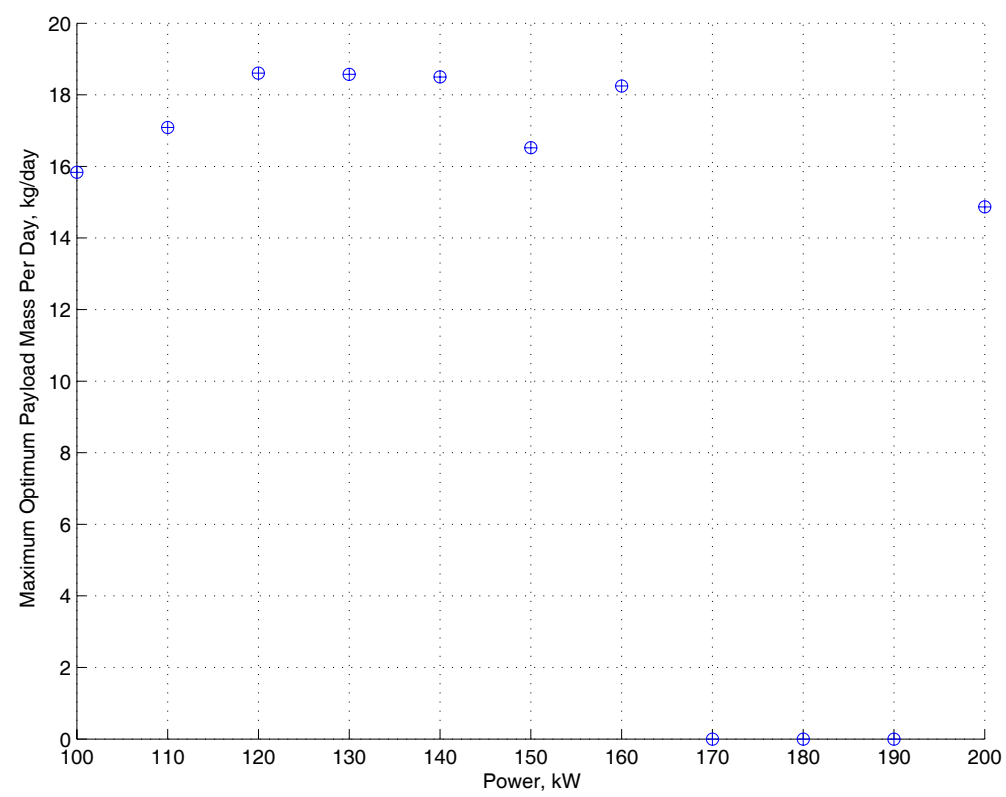

Figure 3.44: Initial Mass Diminishing Returns as a Function of Power

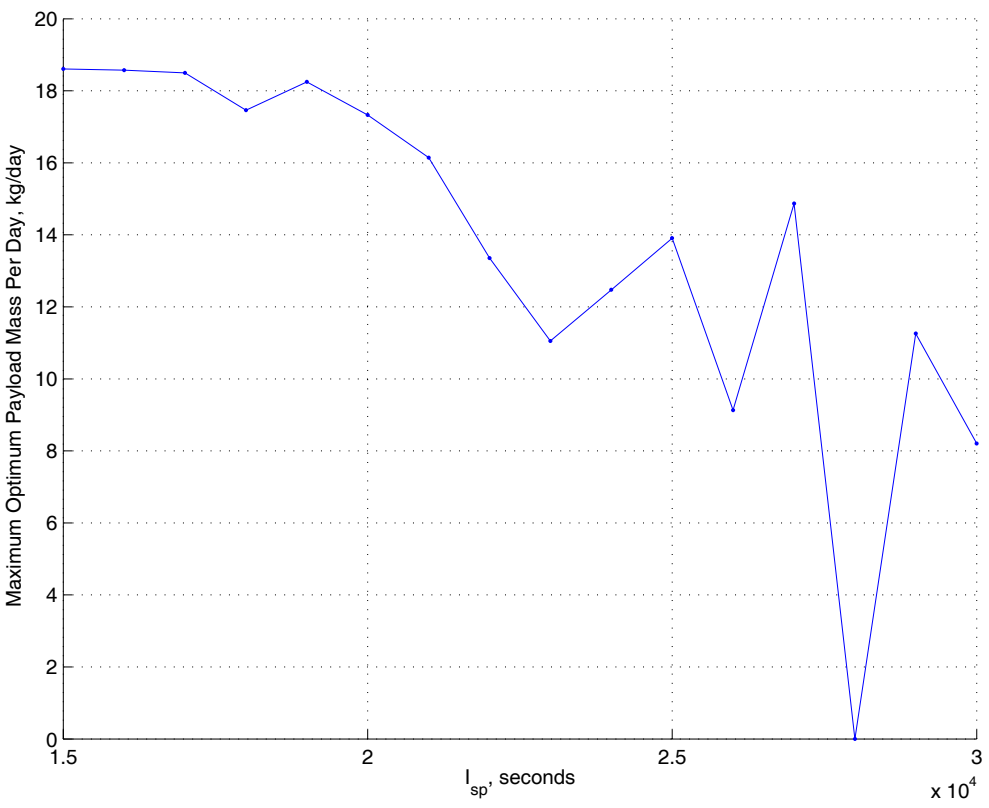

Figure 3.45: Initial Mass Diminishing Returns as a Function of Specific Impulse 


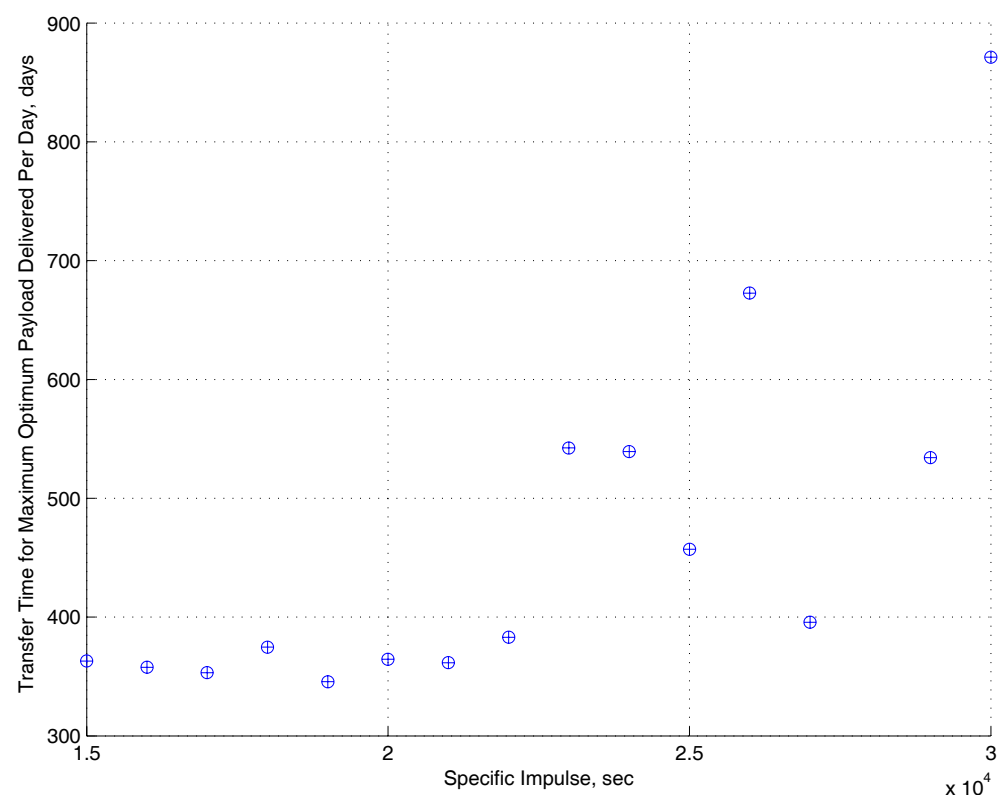

Figure 3.46: Transfer Time as a Function of Specific Impulse for the Maximum Optimum

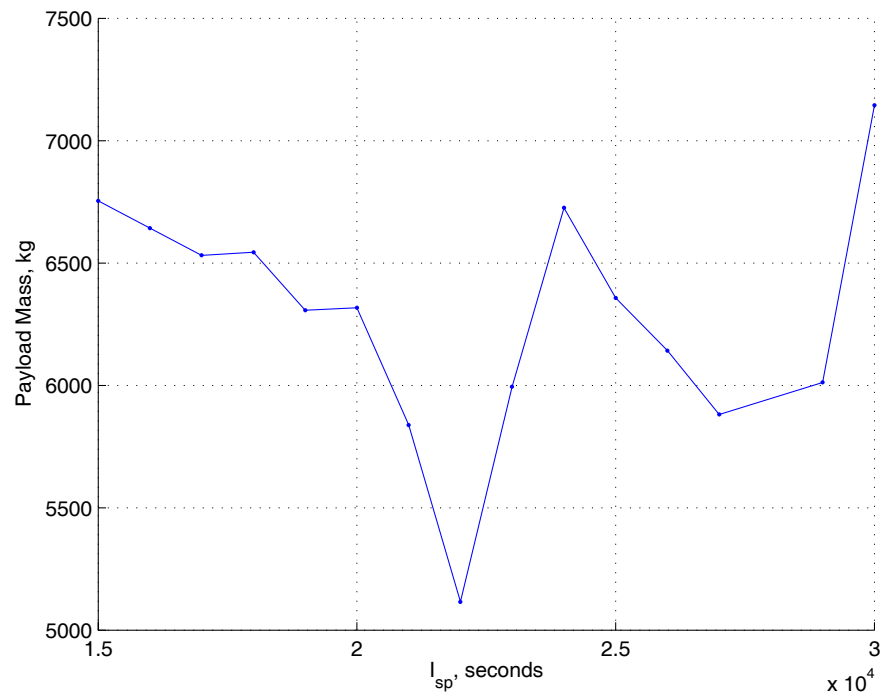

Figure 3.47: Specific impulse vs. Optimum Payload Mass 


\subsection{Discussions}

\subsubsection{Sensitivities}

As can be seen in Figures 3.5 and 3.6, the transfer time is very sensitive to the power used, this can also be seen in Figure 3.49. We can make a comparison of the sensitivity of the transfer time due to power and due to specific impulse, Figures 3.48 and 3.49. These show the transfer time and it can be seen that the power has a greater effect than the specific impulse. Where the variation in specific impulse effects the time of flight by only three days, for a $30 \mathrm{~kW}$ power and $500 \mathrm{~kg}$ initial mass, variations of twenty or more days are present due to power. However at higher power levels further increasing the power will not produce the same effects. This is clear as seen in the previous figure.

Figure 3.17 is also helpful; we can see how the initial mass and the power level affects the payload mass. The $15 \mathrm{~kW}$ power level outperforms the $30 \mathrm{~kW}$ power level by payload mass alone and it is obvious that by increasing the initial mass, the payload mass will follow. Figure 3.18 is a plot of the power level versus payload mass; as the power level increases, the payload mass for a given initial mass and specific impulse decreases. This occurs since the powerplant mass increases as the power level does. You can also see that increasing the specific impulse affects the higher initial masses more than low initial masses; the difference in payload mass due to tripling the specific impulse is proportional to the increase of the initial mass. The difference in payload mass due to specific impulse is about $450 \mathrm{~kg}$ for the $4000 \mathrm{~kg}$ initial mass and about $900 \mathrm{~kg}$ for the $8000 \mathrm{~kg}$ initial mass.

The sensitivity of the payload mass to the power and specific impulse are fairly evident in Figures 3.1 and 3.2. It can also be seen in Figure 3.15; the top spread is about $2000 \mathrm{~kg}$ due to specific impulse where the variation in power results in a spread of nearly $5000 \mathrm{~kg}$.

Mentioned earlier were the assumptions in the mass models for guidance, navigation and control (GNC) and telecommunications systems. The assumption was made that these are small masses and fixed. Historically these systems are small and the assumptions will not impact the overall results significantly. 

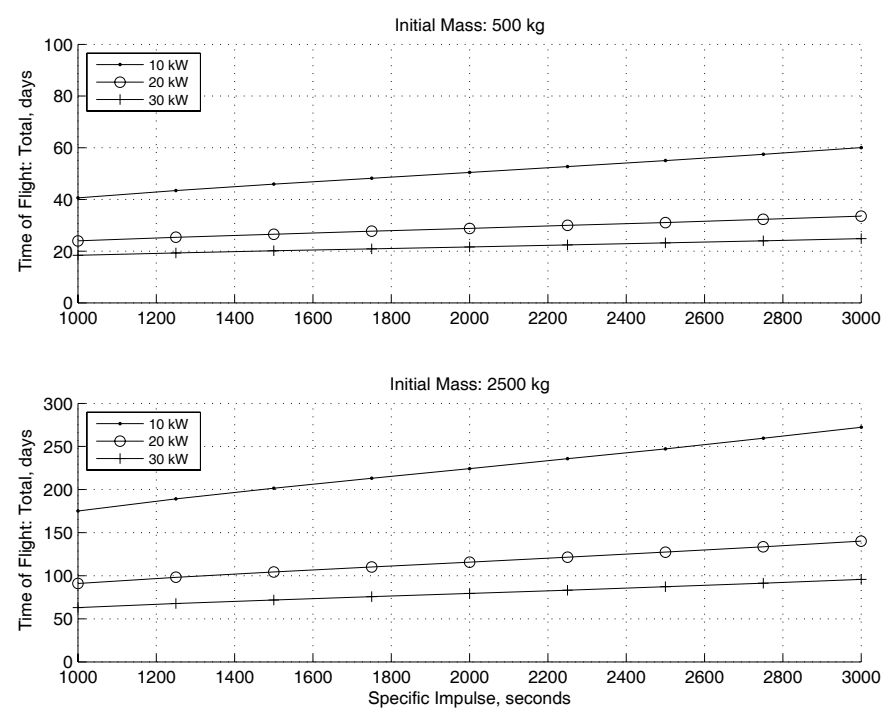

Figure 3.48: Transfer Time as a Function of Specific Impulse
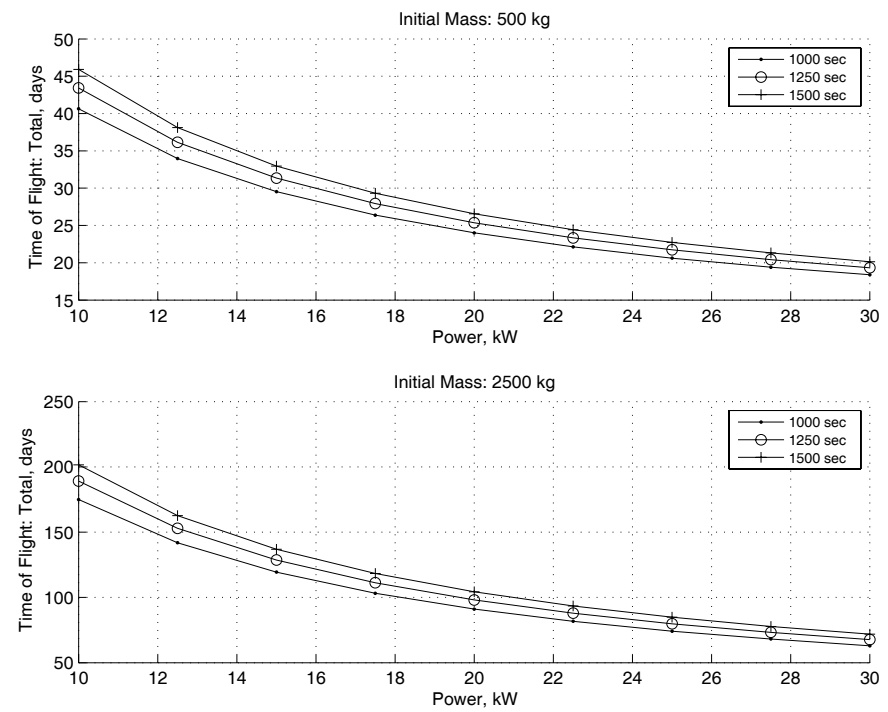

Figure 3.49: Transfer Time as a Function of Power 


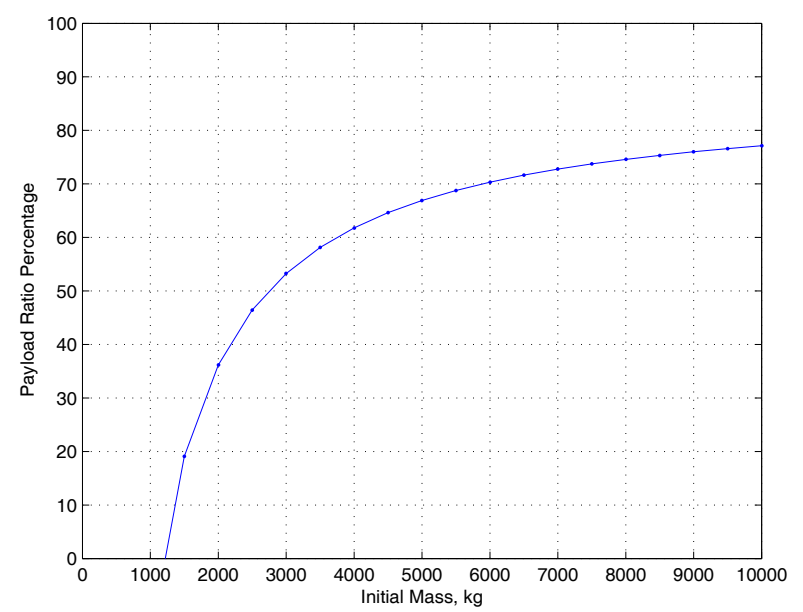

Figure 3.50: Payload Ratio Percentage as a Function of Initial Mass

\subsubsection{Initial Orbit Considerations}

Throughout this study the initial orbit has been assumed to be a $185 \mathrm{~km} \times 35,786 \mathrm{~km}$ GTO at an inclination of $28.5^{\circ}$. It is worth considering the effect of the initial orbit and the spacecraft's stay time in Earth's sphere of influence. Here we study a set of circular initial orbits ranging in altitude from $200 \mathrm{~km}$ to $36,000 \mathrm{~km}$ for a particular power of $50 \mathrm{~kW}$, a specific impulse of 2500 seconds, and an initial mass of $5000 \mathrm{~kg}$. We have already mentioned a few payload ratios when comparing against the baseline RPS. For the near term technology, payload ratios as high as $83.5 \%$ are achievable; using mid term technology, payload ratios as high as $89.5 \%$ are achievable. For this particular combination of inputs, the mid term technology can achieve a payload ratio around $77 \%$, Figure 3.50 .

Figure 3.51 presents the payload mass ratio percentage as a function of initial circular orbit altitude. Clearly, the result is poorer performance when compared to the baseline GTO, reaching not even $68 \%$ at an altitude of $36,000 \mathrm{~km}$.

Figure 3.52 presents the payload mass as a function of initial circular orbit altitude. At $5000 \mathrm{~kg}$ initial mass, the payload mass delivered is similar to the GTO baseline orbit. The GTO baseline will allow about $3345 \mathrm{~kg}$ of payload; a circular orbit of 


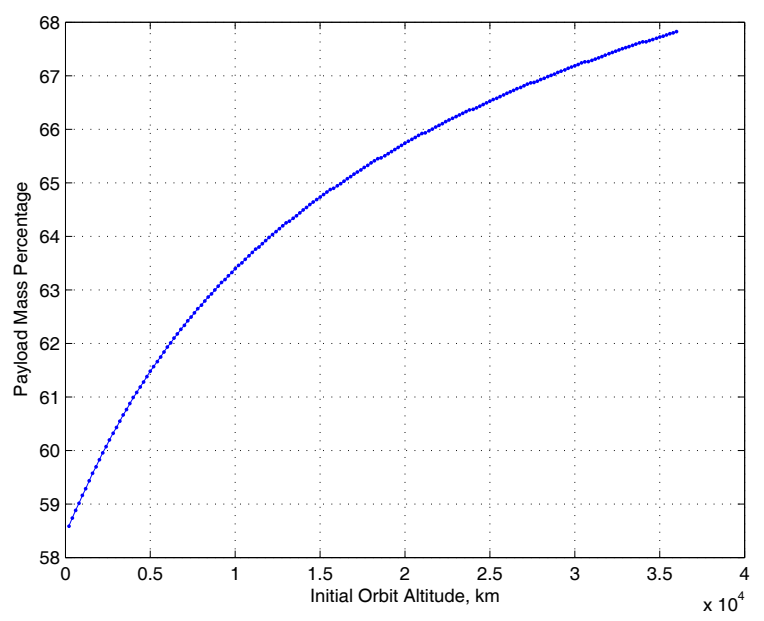

Figure 3.51: Payload Mass Percentage as a Function of Initial Orbit Altitude

approximately $27,500 \mathrm{~km}$ will allow a similar about of payload.

\subsubsection{Technology Level Considerations}

Here we will take a moment to compare the three levels of technology investigated. Looking at Figures 3.54, 3.55, and 3.56 we can see, despite the increasing power levels, that as the specific impulse increases the minimum bound constraining the initial mass grows tighter. The specific impulse has a much greater effect on the transfer time and hence the maximum bound. It is interesting to note that the largest initial mass is transferred using the mid term technology. This is most likely due to the assumption that the thruster efficiency, around a specific impulse of 4500 seconds, levels off. Only modest gains are made when upgrading to far term technology. The maximum payload masses achieved are shown in Figures 3.57, 3.58, and 3.59.

An ERPS system can achieve superior lunar transfer results when compared to a conventional RPS system for payload delivery to lunar orbit. More massive payloads can be delivered and higher ratios of payload delivered per day can be achieved. A combination of specific impulse, initial mass, and power level can be found that maximizes the payload delivered per day for some cases in the trade space. By maximizing 


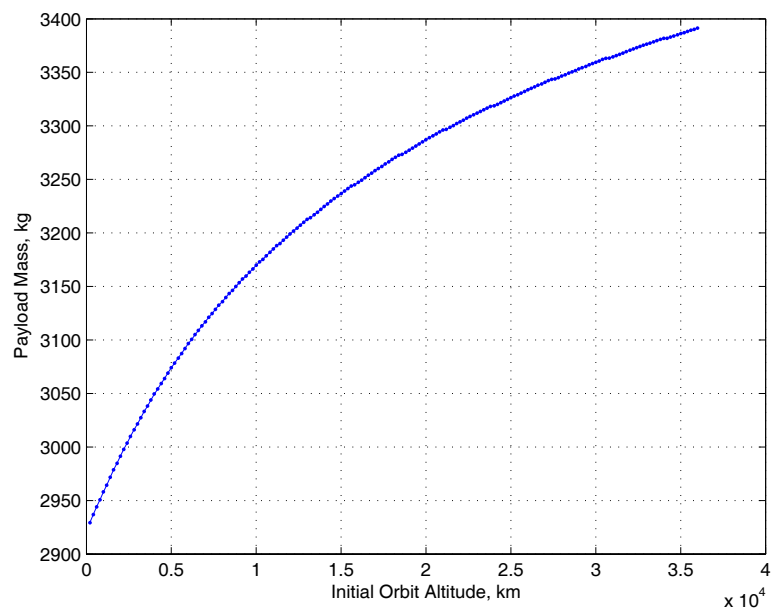

Figure 3.52: Payload Mass as a Function of Initial Orbit Altitude

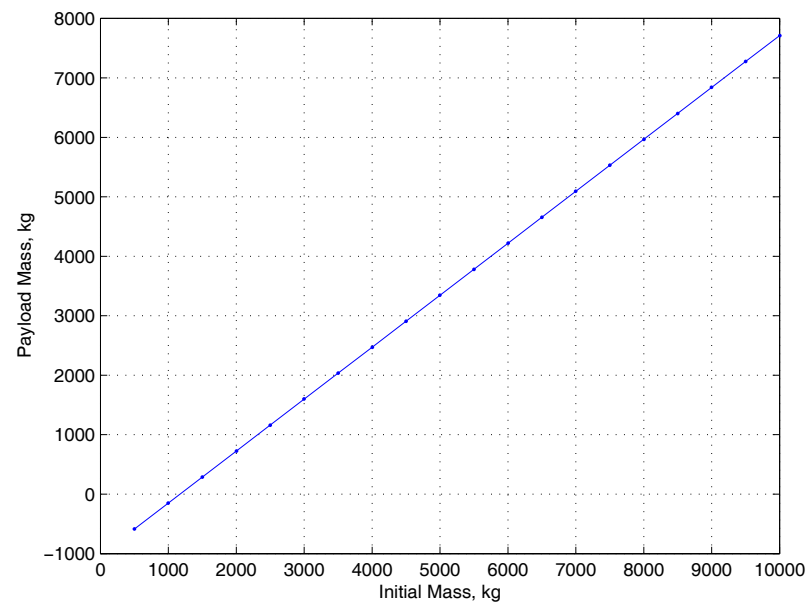

Figure 3.53: Payload Mass as a Function of Initial Mass 


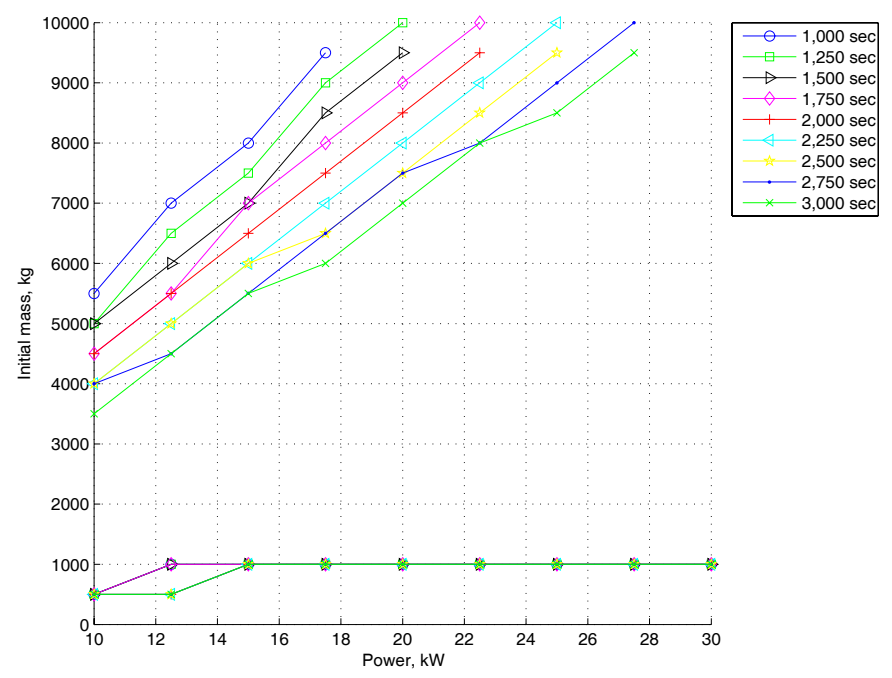

Figure 3.54: Constrained Initial Mass for Near Term Technology

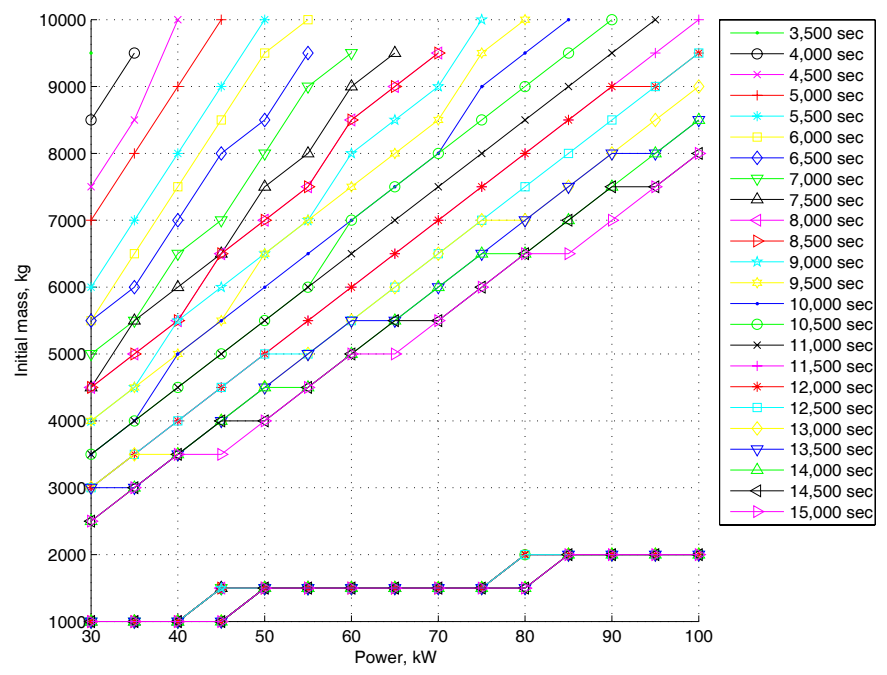

Figure 3.55: Constrained Initial Mass for Mid Term Technology 


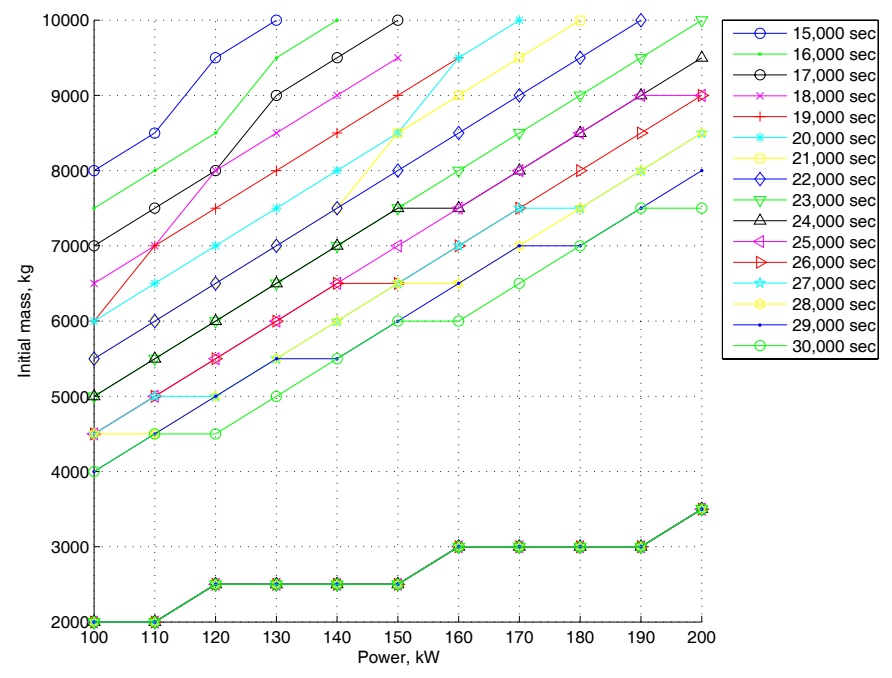

Figure 3.56: Constrained Initial Mass for Far Term Technology

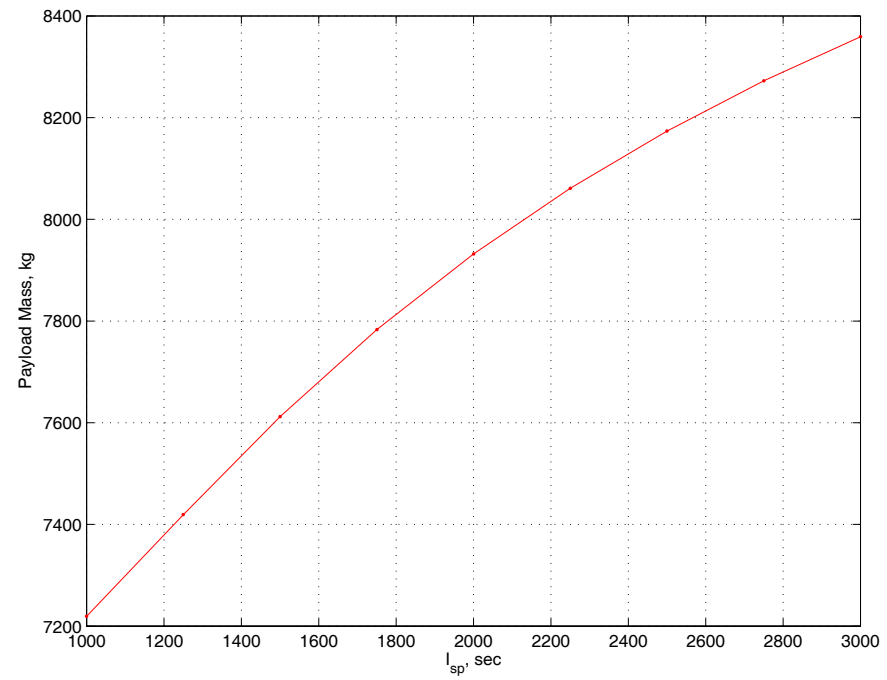

Figure 3.57: Maximum Payload Mass for Near Term Technology 


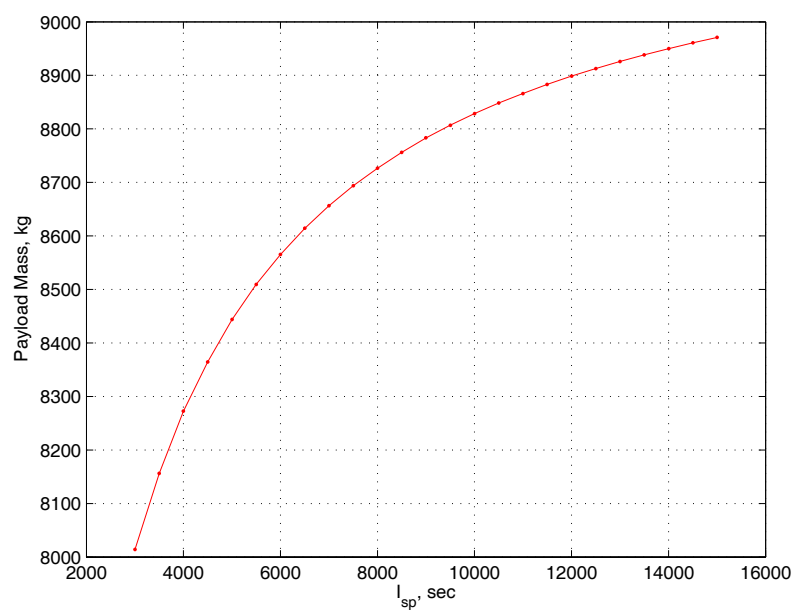

Figure 3.58: Maximum Payload Mass for Mid Term Technology

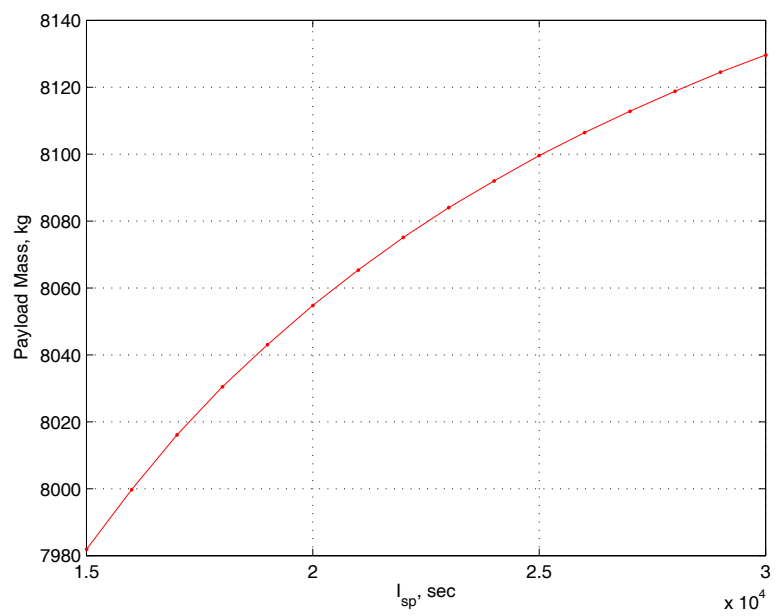

Figure 3.59: Maximum Payload Mass for Far Term Technology 
this ratio, we consider the transfer time as well as the amount of payload delivered. This combination does not necessarily result in the largest delivered payload; despite this, advantage is to be gained. For the Delta II launch vehicle example, the baseline RPS has a payload mass of approximately $250 \mathrm{~kg}$ whereas the near term technology ERPS can be approximately $1126 \mathrm{~kg}$. This mass margin allows many types of missions not previously available and more science to be performed. This increase in capability does come at the expected price of longer transfer times. For the Delta II example the transfer time is about 77 days. However, the objectives investigated are non-time critical and limited to under one year.

\subsubsection{Overall Program Goals}

Our results show that at an initial mass of $2000 \mathrm{~kg}, 20 \mathrm{~kW}$ power level, and specific impulse of 1500 seconds a payload mass of about $1046 \mathrm{~kg}$ can be achieved while a comparably sized RPS, $2100 \mathrm{~kg}$ initial mass, can only achieve a payload mass of $252 \mathrm{~kg}$. The ERPS system is able to deliver over four times as much payload for this example of a Delta II launch vehicle. The second RLEP mission is expected to be a larger lander and may launch on a Delta IV Medium[18]. This launch vehicle has the capability to put $3960 \mathrm{~kg}$ into GTO. The RPS can place roughly $278 \mathrm{~kg}$ of payload into LLO. An equivalent ERPS with an initial mass of roughly $4000 \mathrm{~kg}(20 \mathrm{~kW}$ power and $1500 \mathrm{~s} I_{s p}$ ) can place $2860 \mathrm{~kg}$ of payload into LLO, more than ten times as much payload.

Knowing this, we can easily conceive of a plan for an SEP to deliver multiple RLEP missions, say an orbiter and a lander, from one launch vehicle. Such a capability could shorten the baseline RLEP timeline and reduce launch costs or allow more missions to be launched if the baseline schedule were to be continued. The assumed schedule

for the baseline RLEP is a mission launch every two years. LRO is scheduled to launch in 2008 (estimated here at $250 \mathrm{~kg}$ ) and the second RLEP mission in 2010 (estimated here at $700 \mathrm{~kg}$ ). It is clearly feasible that an ERPS can deliver a lander, an enabling technology for the second planned RLEP mission, on a smaller launch vehicle or paired with another RLEP mission on the originally planned launch vehicle. 
Figure 3.60 shows a notional comparison between the assumed baseline schedule of a launch every two years with a schedule enabled by the ERPS. The ERPS schedule assumes that the launch vehicle is a Delta II and that each launch is a pairing of an orbiter and a lander, where the estimated masses of the orbiters are $250 \mathrm{~kg}$ and the landers are $700 \mathrm{~kg}$. It is clear that an ERPS can enable RLEP missions to launch on small vehicles like the Delta II as well as requiring fewer launches.

Assuming the Delta II launch vehicle and a launch cost of $\$ 80 \mathrm{M}$, the first six missions can be launched at a cost of $\$ 240 \mathrm{M}$. Using conventional chemical propulsion it would be six launches, costing around $\$ 480 \mathrm{M}$. Estimates of the SEP stage[23] put the cost of a $98 \mathrm{~kW}$ SEP tug around $\$ 44 \mathrm{M}$ while a smaller SEP tug as discussed about would cost closer to $\$ 10 \mathrm{M}$. Three SEP stages are needed for the first six missions. There is then about a $\$ 200 \mathrm{M}$ savings as compared to the RLEP baseline.

Once the payload has been delivered to the lunar infrastructure, the SEP stage will still be in orbit about the moon. The first thought that jumps to mind for its use is as a communications platform. The SEP stage will be fairly high powered, upward of $10 \mathrm{~kW}$, and will already have a communications package for operations. This is an easy way to extend its usefulness as well as extending capability for the investment. Problems relating to an optimum lunar orbit for such activity can be addressed in future work. Of course some of the payload could be offloaded into instruments that remain aboard the SEP stage and add yet more value to post-primary mission activities.

Technology demonstration is yet another way in which a SEP spacecraft can contribute to the overall RLEP goals; electric propulsion is continually demonstrated in Earth orbit on GEO communications satellites[24] and in the past for lunar mission through SMART-1[25]. Electric propulsion can achieve very promising results for human-scale missions[23] and beginning to fly this technology early will gain much needed heritage for future lunar and Mars missions. 


\section{A RLEP Mission Timeline Comparison}

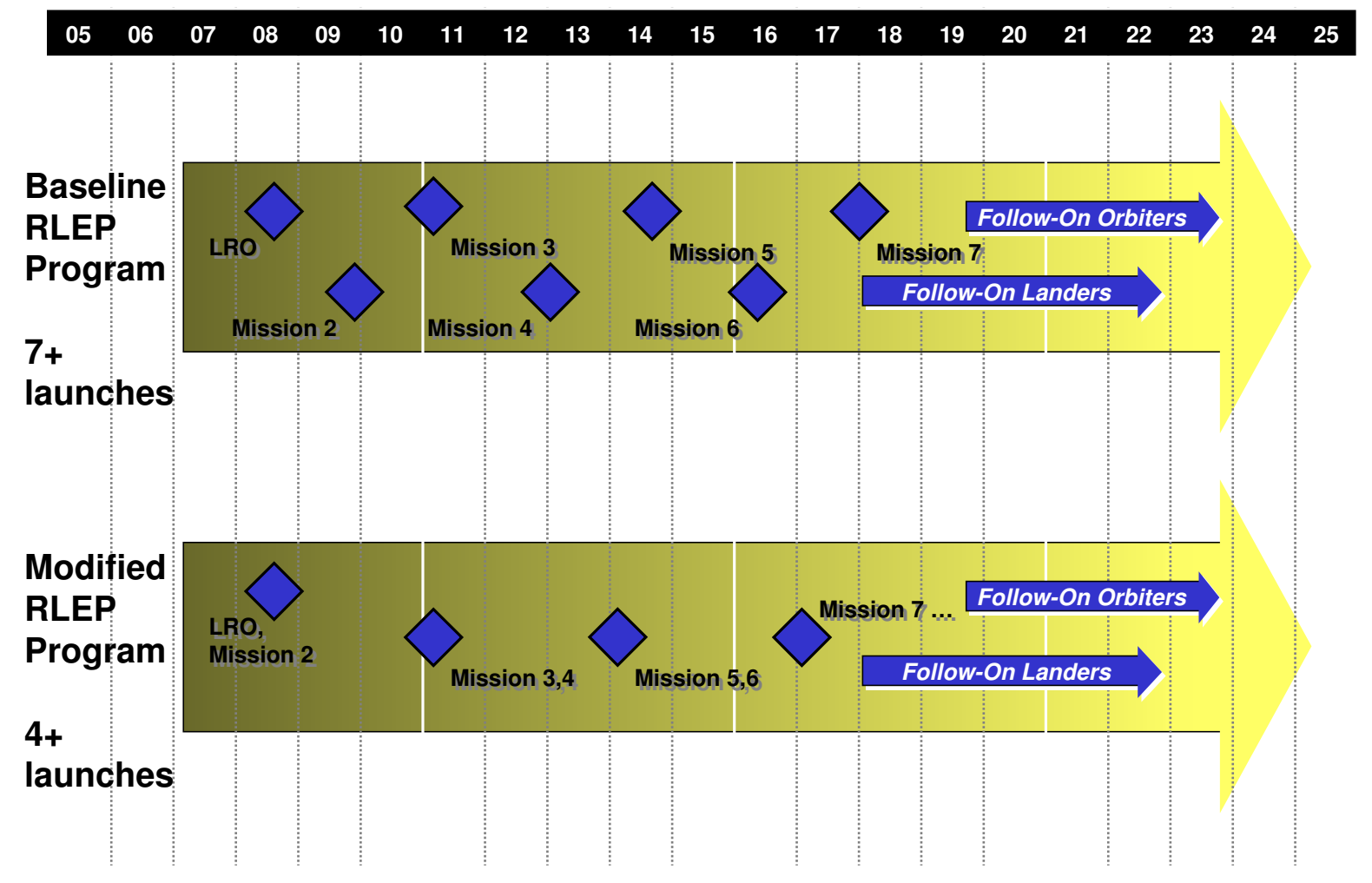

Figure 3.60: RLEP Schedule Comparison 


\section{Chapter 4}

\section{Summary and Conclusions}

This work has investigated a large trade space of possible SEP vehicles in the Earth lunar system. Straightforward output parameters such as payload mass and transfer times have been calculated for some 18,000 cases. Sections of the trade space where a point of diminished returns is reached have also been found. The resultant space is analyzed to find what combinations of specific impulse, initial mass, and power levels provide the largest payload masses in LLO and which are the most efficient in terms of a transportation rate.

For the near term application investigated, ERPS using Hall thrusters can provide an important function delivering payloads to the moon that will be needed for the Robotic Lunar Precursor Program. The capability is also large enough to enable early manned support missions, delivering rovers to aid astronauts for example. It is also able to allow the use of small launch vehicles as well as fewer, directly enabling launch cost savings.

Our results show that at an initial mass of $2000 \mathrm{~kg}, 20 \mathrm{~kW}$ power level, and specific impulse of 1500 seconds a payload mass of about $1046 \mathrm{~kg}$ can be achieved, a payload mass ratio of $52.28 \%$. A comparably sized RPS, $2100 \mathrm{~kg}$ initial mass, can only achieve a payload mass of $252 \mathrm{~kg}$, a payload mass ratio of only $12.0 \%$. The benefit of electric propulsion is a payload mass increase of $794 \mathrm{~kg}$.

If the specific impulse is increased to 2500 seconds then a payload mass of about $1158 \mathrm{~kg}$ can be achieved, a payload mass ratio of $57.90 \%$. If the initial mass constraint 
is increased to $4000 \mathrm{~kg}$ the benefit of electric propulsion would be an additional $1710 \mathrm{~kg}$ of payload, at a mass ratio of $71.70 \%$.

Besides cost savings an SEP system affords flexibility in the number and scheduling of launches. The end result is a flexible SEP system that can deliver more mass to LLO than conventional chemical propulsion while saving an estimated $\$ 200 \mathrm{M}$.

\subsection{Recommendation for Future Work}

Future studies should further investigate the nature of thruster efficiency for high specific impulse thrusters. The assumption made in this study concerning specific impulse showed a large effect and mitigated gains for far term technology applications.

The Delta II launch vehicle was assumed for near term technology levels since it is an available and proven vehicle and the first RLEP mission, LRO, is planning to use it. It was shown that the ERPS can deliver much more payload to LLO so it is advisable that other launch vehicles be considered. The program cost and schedule would need to be revisited following such consideration.

Also, more in depth assessment of a follow on mission capability, such as providing communications or power for future missions, may need to be performed. A further investigation of possible payloads might be helpful in refining the requirements. This could lead to further refinement of the launch vehicle selection and scheduling. 


\section{Appendix A}

\section{Appendix: Overall Plots}

The following plots, Figures A.1 to A.2, present the initial mass versus payload mass in low lunar orbit for power levels from $10 \mathrm{~kW}$ to $200 \mathrm{~kW}$.
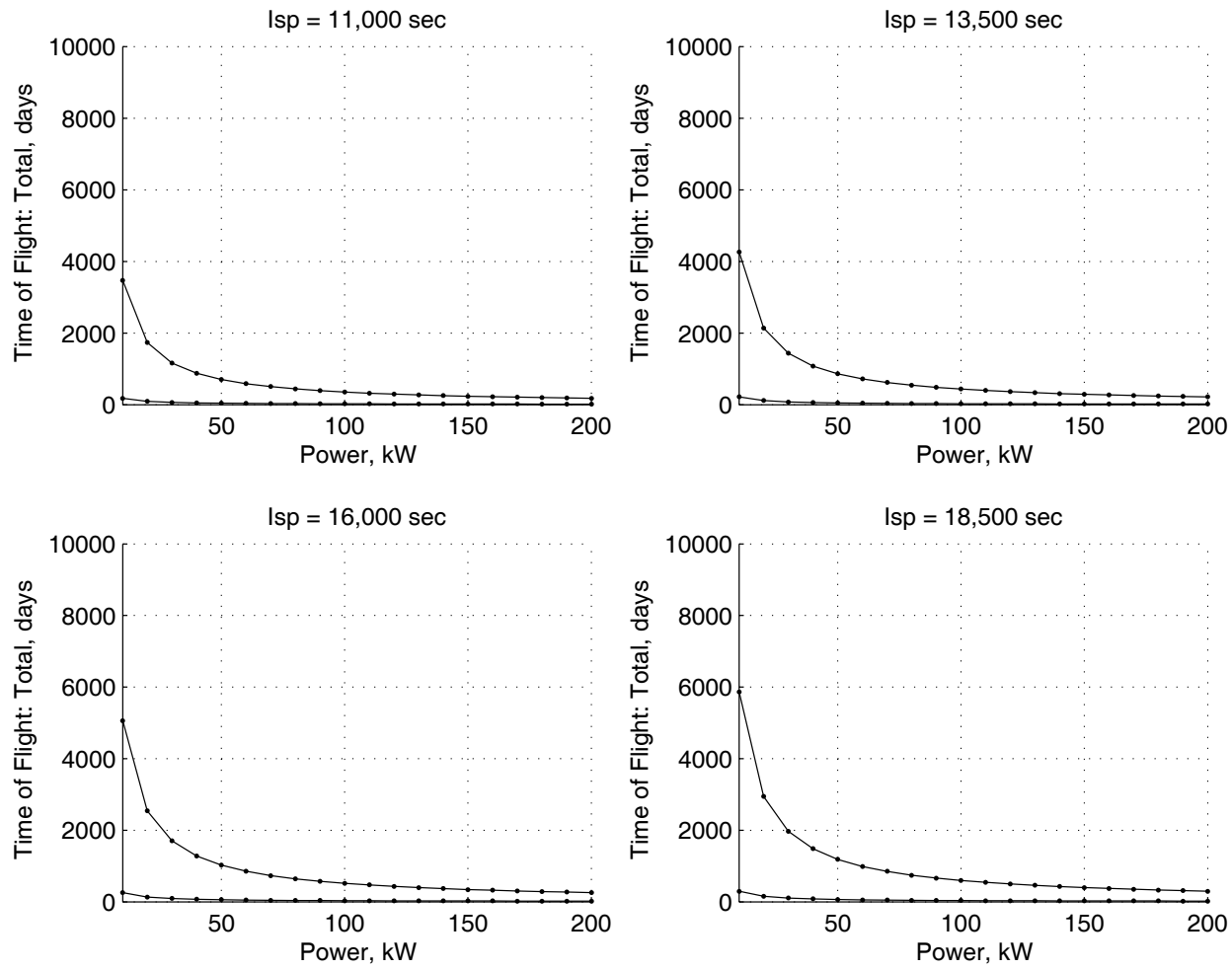

Figure A.1: Power vs. Total Time of Flight, Overall 

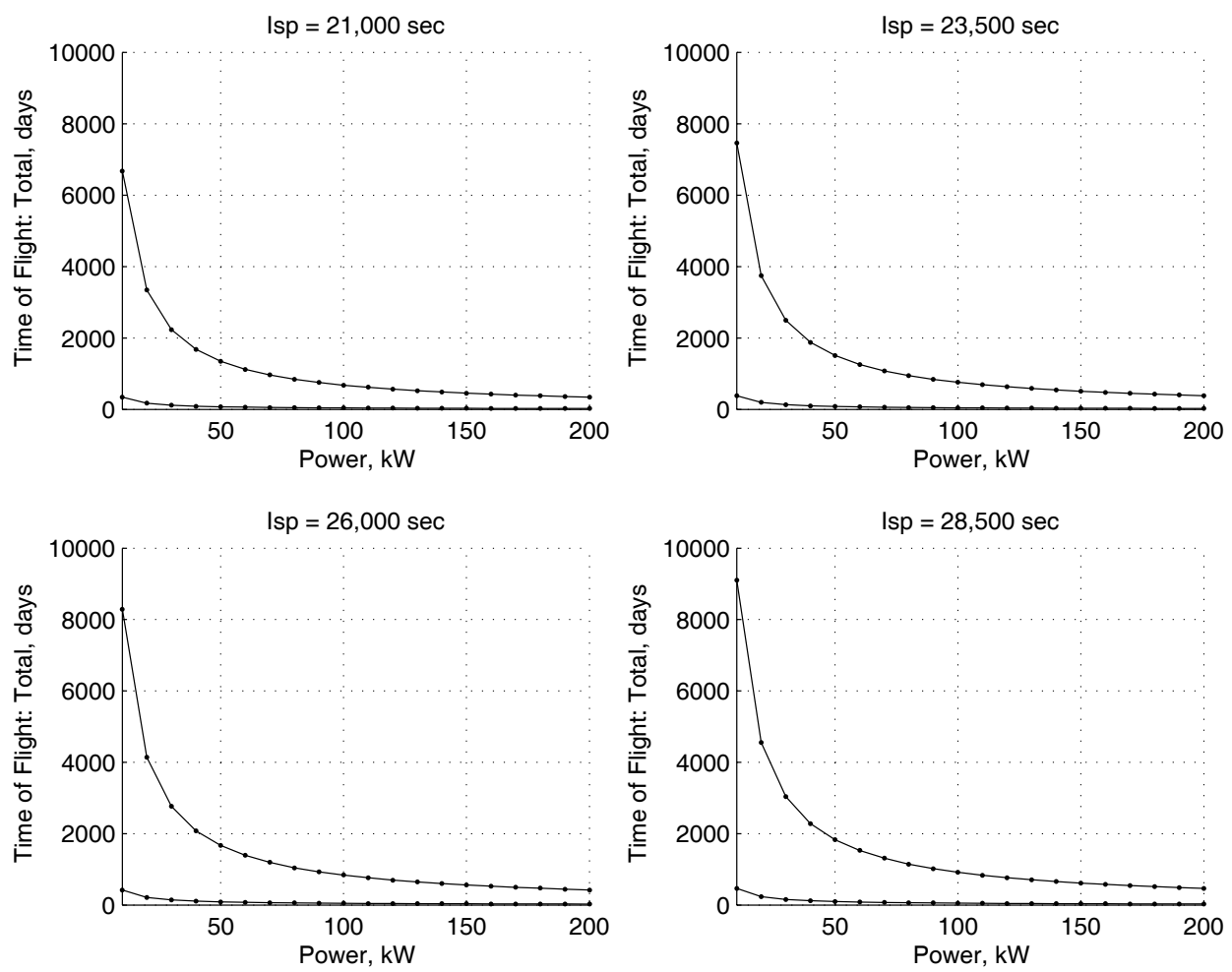

Figure A.2: Power vs. Total Time of Flight, Overall 
The following plots, Figures A.3 to A.4, present the initial mass versus the transfer time split to show each specific impulse separately.
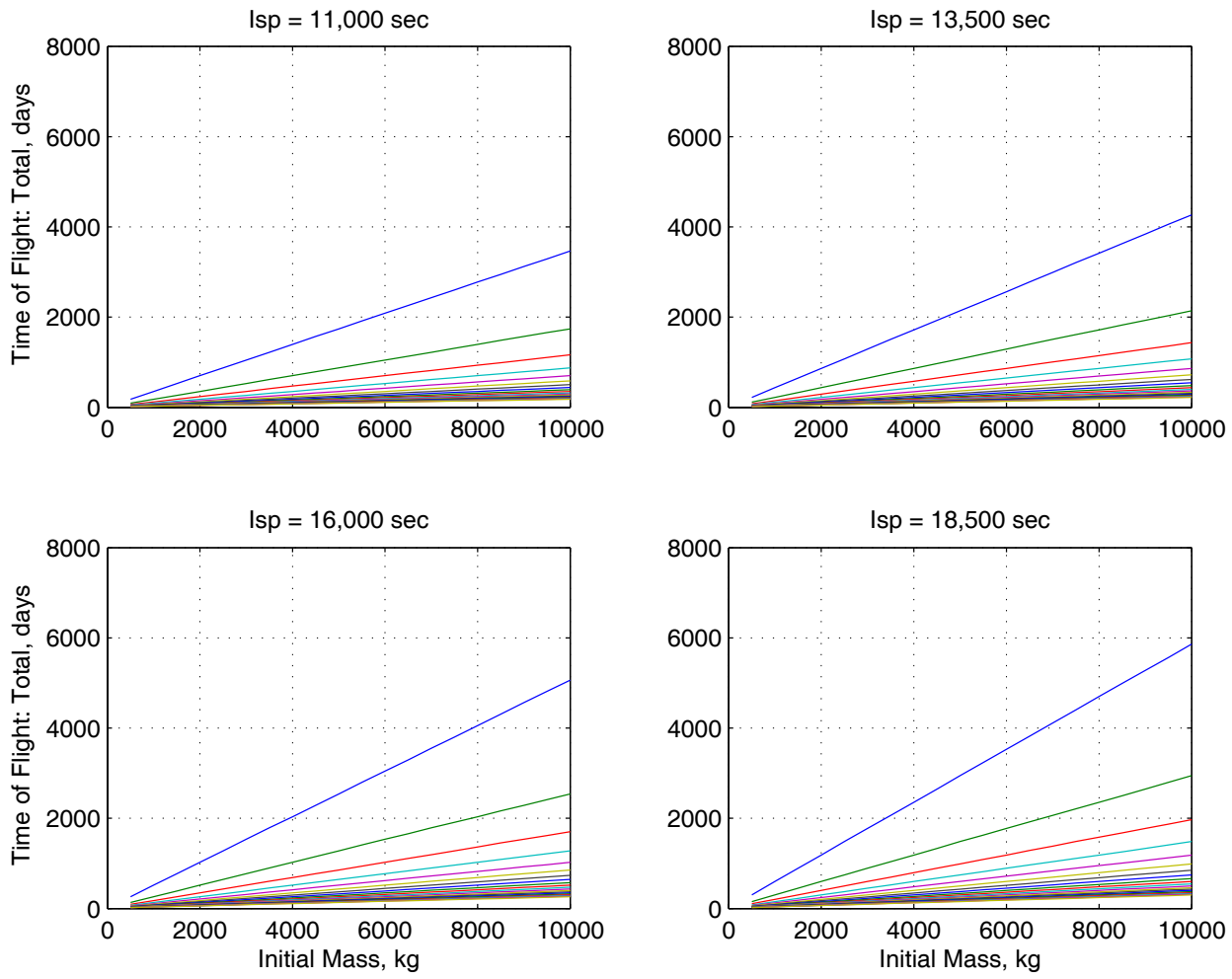

Figure A.3: Initial Mass vs. Total Time of Flight, Overall 

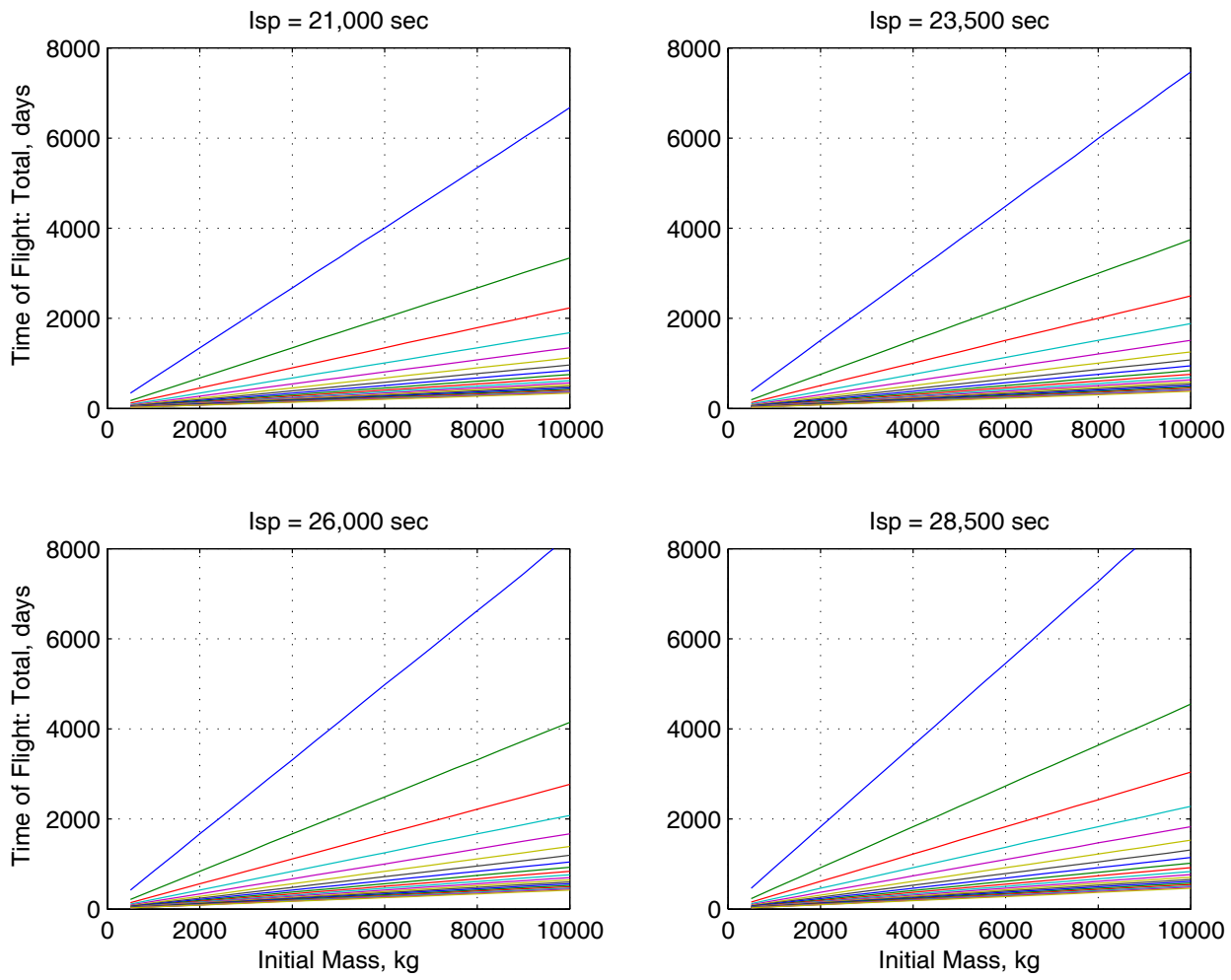

Figure A.4: Initial Mass vs. Total Time of Flight, Overall 
Figures A.5 to A.6, presents the payload mass delivered per day.
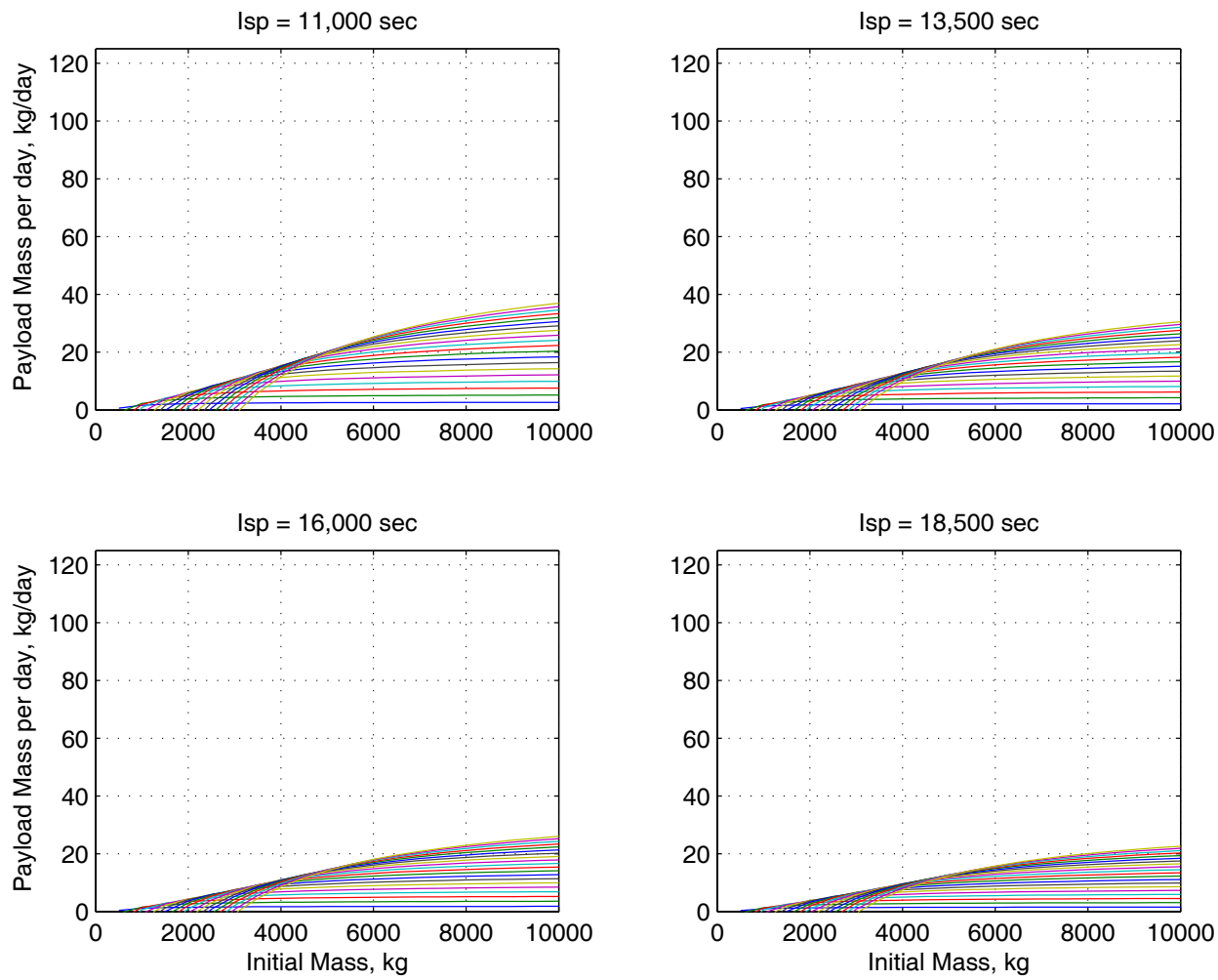

Figure A.5: Initial Mass vs. Payload Mass per Day, Overall 

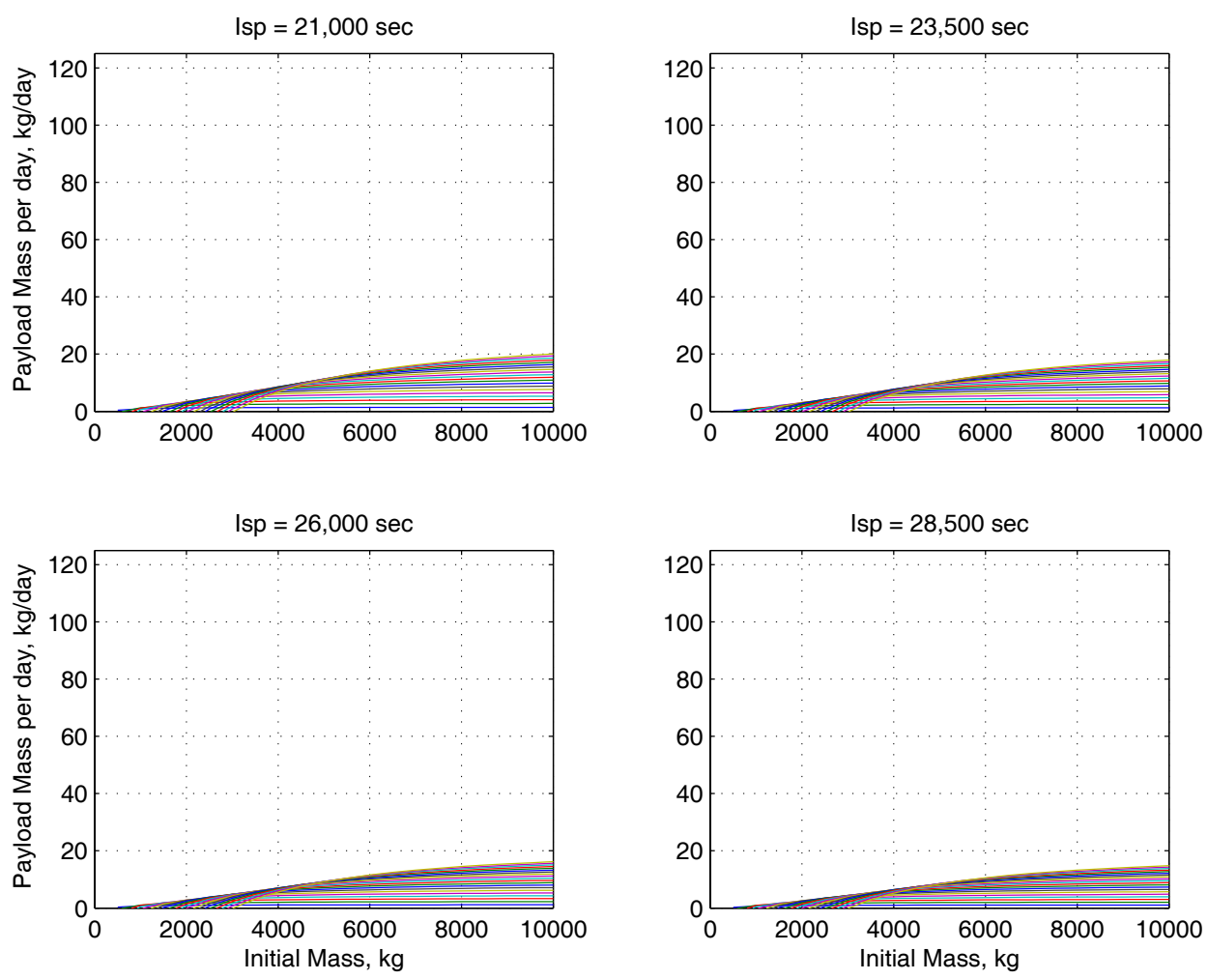

Figure A.6: Initial Mass vs. Payload Mass per Day, Overall 
The following are the tabulated results for the points of diminishing returns, where one exists for a combination of initial mass, power, and specific impulse, sorted by power. There are 98 cases that have a point of diminishing returns for the initial mass versus payload delivered per day curves.

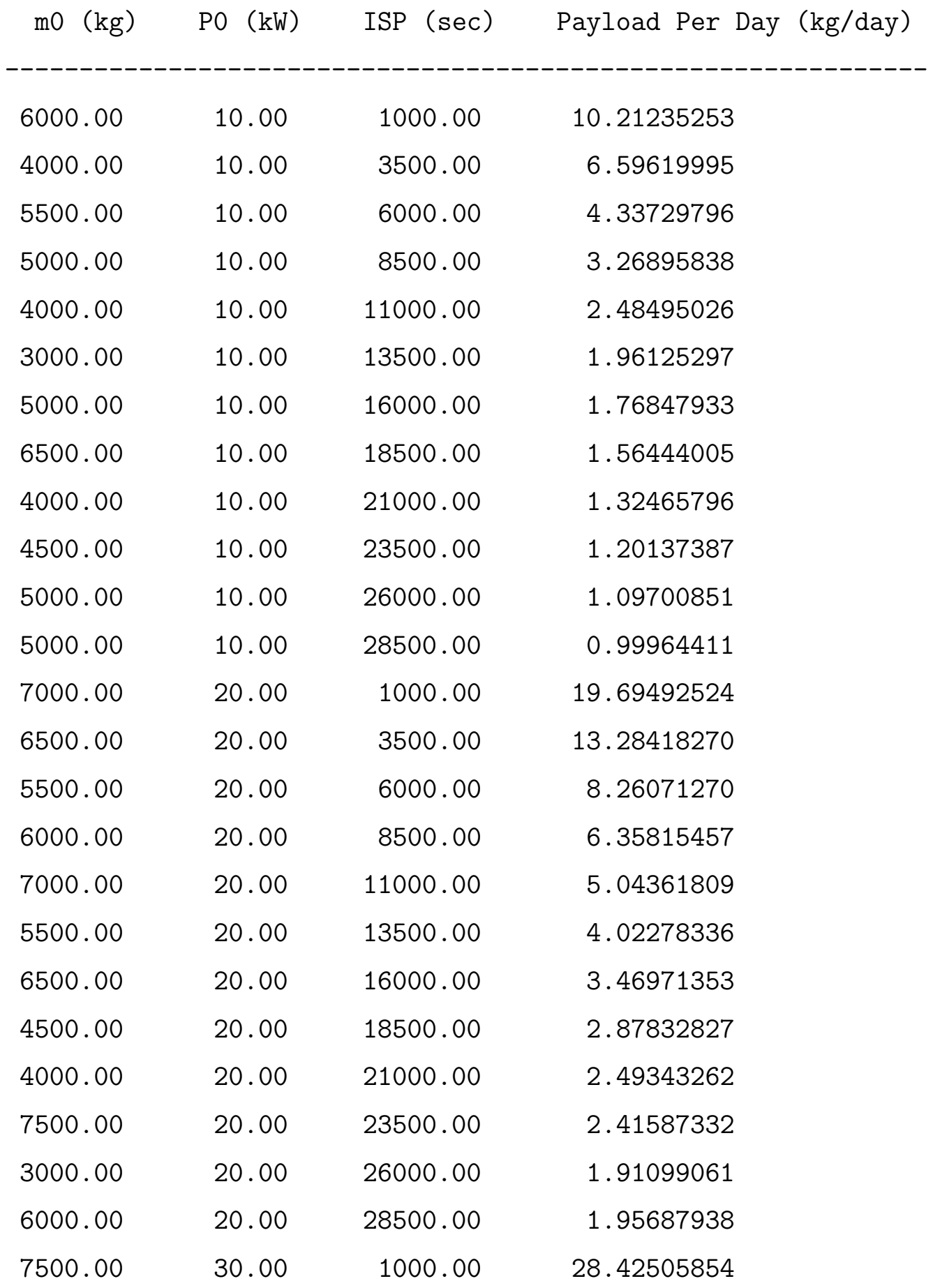




$\begin{array}{rrrr}8500.00 & 30.00 & 3500.00 & 20.01228621 \\ 7000.00 & 30.00 & 6000.00 & 12.32509269 \\ 8000.00 & 30.00 & 8500.00 & 9.54186965 \\ 7000.00 & 30.00 & 11000.00 & 7.32032424 \\ 6000.00 & 30.00 & 13500.00 & 5.88443297 \\ 7000.00 & 30.00 & 16000.00 & 5.09124856 \\ 6000.00 & 30.00 & 18500.00 & 4.32801335 \\ 6000.00 & 30.00 & 21000.00 & 3.82146280 \\ 5500.00 & 30.00 & 23500.00 & 3.37604719 \\ 6000.00 & 30.00 & 26000.00 & 3.09763258 \\ 4500.00 & 30.00 & 28500.00 & 2.69621316 \\ 8500.00 & 40.00 & 1000.00 & 37.16912920 \\ 7500.00 & 40.00 & 3500.00 & 25.37035181 \\ 7000.00 & 40.00 & 6000.00 & 16.04366841 \\ 5500.00 & 40.00 & 8500.00 & 11.62875244 \\ 6500.00 & 40.00 & 11000.00 & 9.35797587 \\ 8000.00 & 40.00 & 13500.00 & 7.94359873 \\ 6500.00 & 40.00 & 16000.00 & 6.51449348 \\ 7500.00 & 40.00 & 18500.00 & 5.79392719 \\ 8000.00 & 40.00 & 21000.00 & 5.15786660 \\ 7500.00 & 40.00 & 23500.00 & 4.57266536 \\ 6500.00 & 40.00 & 26000.00 & 4.05515046 \\ 6000.00 & 40.00 & 28500.00 & 3.65627953 \\ 8000.00 & 50.00 & 3500.00 & 31.09072491 \\ 9000.00 & 50.00 & 6000.00 & 20.29533875 \\ 7000.00 & 50.00 & 8500.00 & 14.77025818 \\ 6500.00 & 50.00 & 11000.00 & 11.34195358 \\ 9000.00 & 50.00 & 13500.00 & 9.85899109 \\ 8500.00 & 50.00 & 16000.00 & 8.29009408 \\ 8500.00 & 50.00 & 21000.00 & 6.36187824 \\ 7500.00 & 50.00 & 23500.00 & 5.58856719\end{array}$




\begin{tabular}{rrrr}
6500.00 & 50.00 & 26000.00 & 4.92922075 \\
6500.00 & 50.00 & 28500.00 & 4.50023844 \\
9000.00 & 60.00 & 6000.00 & 23.87791790 \\
8500.00 & 60.00 & 8500.00 & 17.91547748 \\
6500.00 & 60.00 & 11000.00 & 13.22636220 \\
8500.00 & 60.00 & 13500.00 & 11.46769850 \\
8000.00 & 60.00 & 16000.00 & 9.62838817 \\
8500.00 & 60.00 & 18500.00 & 8.44839829 \\
7500.00 & 60.00 & 21000.00 & 7.30051728 \\
9000.00 & 60.00 & 23500.00 & 6.76060423 \\
8000.00 & 60.00 & 26000.00 & 6.00229750 \\
8000.00 & 60.00 & 28500.00 & 5.47378290 \\
8000.00 & 70.00 & 6000.00 & 26.53643078 \\
7500.00 & 70.00 & 11000.00 & 15.53284326 \\
8000.00 & 70.00 & 16000.00 & 10.96538262 \\
9000.00 & 70.00 & 18500.00 & 9.75247642 \\
7000.00 & 70.00 & 21000.00 & 8.16784728 \\
8000.00 & 70.00 & 23500.00 & 7.55303595 \\
8000.00 & 80.00 & 3500.00 & 45.32291227 \\
9000.00 & 80.00 & 8500.00 & 23.24792133 \\
8000.00 & 80.00 & 11000.00 & 17.66222305 \\
7500.00 & 80.00 & 28500.00 & 6.88067491 \\
7000.00 & 90.00 & 11000.00 & 18.69782759 \\
8500.00 & 90.00 & 28500.00 & 7.80733896 \\
9000.00 & 100.00 & 13500.00 & 17.80632384 \\
7500.00 & 100.00 & 16000.00 & 14.33637632 \\
8000.00 & 100.00 & 26000.00 & 9.13238262 \\
8500.00 & 110.00 & 16000.00 & 16.02705369 \\
7000.00 & 110.00 & 18500.00 & 13.04839557 \\
8500.00 & 110.00 & 21000.00 & 12.30034729 \\
\hline 9000.00 & 120.00 & 16000.00 & 17.41860782
\end{tabular}




$\begin{array}{rrrr}9000.00 & 120.00 & 28500.00 & 9.94796644 \\ 9000.00 & 130.00 & 1000.00 & 88.53185285 \\ 9000.00 & 130.00 & 16000.00 & 18.57166874 \\ 7500.00 & 130.00 & 21000.00 & 13.30061496 \\ 9000.00 & 130.00 & 23500.00 & 12.72957221 \\ 8500.00 & 140.00 & 18500.00 & 16.66767196 \\ 8500.00 & 140.00 & 23500.00 & 13.17570879 \\ 8500.00 & 150.00 & 11000.00 & 28.71317620 \\ 8500.00 & 160.00 & 21000.00 & 16.14638280 \\ 9000.00 & 170.00 & 6000.00 & 52.13767398 \\ 9000.00 & 190.00 & 23500.00 & 16.55580066\end{array}$

The following are the tabulated results for the points of diminishing returns, where one exists for a combination of initial mass, power, and specific impulse, sorted by specific impulse.

$\begin{array}{lrrr}\mathrm{m} 0(\mathrm{~kg}) & \text { P0 }(\mathrm{kW}) & \text { ISP }(\mathrm{sec}) & \text { Payload Per Day }(\mathrm{kg} / \mathrm{day}) \\ 6000.00 & 10.00 & 1000.00 & 10.21235253 \\ 7000.00 & 20.00 & 1000.00 & 19.69492524 \\ 7500.00 & 30.00 & 1000.00 & 28.42505854 \\ 8500.00 & 40.00 & 1000.00 & 37.16912920 \\ 9000.00 & 130.00 & 1000.00 & 88.53185285 \\ 4000.00 & 10.00 & 3500.00 & 6.59619995 \\ 6500.00 & 20.00 & 3500.00 & 13.28418270 \\ 8500.00 & 30.00 & 3500.00 & 20.01228621 \\ 7500.00 & 40.00 & 3500.00 & 25.37035181 \\ 8000.00 & 50.00 & 3500.00 & 31.09072491 \\ 8000.00 & 80.00 & 3500.00 & 45.32291227 \\ 5500.00 & 10.00 & 6000.00 & 4.33729796 \\ 5500.00 & 20.00 & 6000.00 & 8.26071270 \\ 7000.00 & 30.00 & 6000.00 & 12.32509269\end{array}$




$\begin{array}{rrrr}7000.00 & 40.00 & 6000.00 & 16.04366841 \\ 9000.00 & 50.00 & 6000.00 & 20.29533875 \\ 9000.00 & 60.00 & 6000.00 & 23.87791790 \\ 8000.00 & 70.00 & 6000.00 & 26.53643078 \\ 9000.00 & 170.00 & 6000.00 & 52.13767398 \\ 5000.00 & 10.00 & 8500.00 & 3.26895838 \\ 6000.00 & 20.00 & 8500.00 & 6.35815457 \\ 8000.00 & 30.00 & 8500.00 & 9.54186965 \\ 5500.00 & 40.00 & 8500.00 & 11.62875244 \\ 7000.00 & 50.00 & 8500.00 & 14.77025818 \\ 8500.00 & 60.00 & 8500.00 & 17.91547748 \\ 9000.00 & 80.00 & 8500.00 & 23.24792133 \\ 4000.00 & 10.00 & 11000.00 & 2.48495026 \\ 7000.00 & 20.00 & 11000.00 & 5.04361809 \\ 7000.00 & 30.00 & 11000.00 & 7.32032424 \\ 6500.00 & 40.00 & 11000.00 & 9.35797587 \\ 6500.00 & 50.00 & 11000.00 & 11.34195358 \\ 6500.00 & 60.00 & 11000.00 & 13.22636220 \\ 7500.00 & 70.00 & 11000.00 & 15.53284326 \\ 8000.00 & 80.00 & 11000.00 & 17.66222305 \\ 7000.00 & 90.00 & 11000.00 & 18.69782759 \\ 8500.00 & 150.00 & 11000.00 & 28.71317620 \\ 3000.00 & 10.00 & 13500.00 & 1.96125297 \\ 5500.00 & 20.00 & 13500.00 & 4.02278336 \\ 6000.00 & 30.00 & 13500.00 & 5.88443297 \\ 8000.00 & 40.00 & 13500.00 & 7.94359873 \\ 9000.00 & 50.00 & 13500.00 & 9.85899109 \\ 8500.00 & 60.00 & 13500.00 & 11.46769850 \\ 9000.00 & 100.00 & 13500.00 & 17.80632384 \\ 5000.00 & 10.00 & 16000.00 & 1.76847933 \\ 6500.00 & 20.00 & 16000.00 & 3.46971353\end{array}$




$\begin{array}{rrrr}7000.00 & 30.00 & 16000.00 & 5.09124856 \\ 6500.00 & 40.00 & 16000.00 & 6.51449348 \\ 8500.00 & 50.00 & 16000.00 & 8.29009408 \\ 8000.00 & 60.00 & 16000.00 & 9.62838817 \\ 8000.00 & 70.00 & 16000.00 & 10.96538262 \\ 7500.00 & 100.00 & 16000.00 & 14.33637632 \\ 8500.00 & 110.00 & 16000.00 & 16.02705369 \\ 9000.00 & 120.00 & 16000.00 & 17.41860782 \\ 9000.00 & 130.00 & 16000.00 & 18.57166874 \\ 6500.00 & 10.00 & 18500.00 & 1.56444005 \\ 4500.00 & 20.00 & 18500.00 & 2.87832827 \\ 6000.00 & 30.00 & 18500.00 & 4.32801335 \\ 7500.00 & 40.00 & 18500.00 & 5.79392719 \\ 8500.00 & 60.00 & 18500.00 & 8.44839829 \\ 9000.00 & 70.00 & 18500.00 & 9.75247642 \\ 7000.00 & 110.00 & 18500.00 & 13.04839557 \\ 8500.00 & 140.00 & 18500.00 & 16.66767196 \\ 4000.00 & 10.00 & 21000.00 & 1.32465796 \\ 4000.00 & 20.00 & 21000.00 & 2.49343262 \\ 6000.00 & 30.00 & 21000.00 & 3.82146280 \\ 8000.00 & 40.00 & 21000.00 & 5.15786660 \\ 8500.00 & 50.00 & 21000.00 & 6.36187824 \\ 7500.00 & 60.00 & 21000.00 & 7.30051728 \\ 7000.00 & 70.00 & 21000.00 & 8.16784728 \\ 8500.00 & 110.00 & 21000.00 & 12.30034729 \\ 7500.00 & 130.00 & 21000.00 & 13.30061496 \\ 8500.00 & 160.00 & 21000.00 & 16.14638280 \\ 4500.00 & 10.00 & 23500.00 & 1.20137387 \\ 7500.00 & 20.00 & 23500.00 & 2.41587332 \\ 5500.00 & 30.00 & 23500.00 & 3.37604719 \\ 7500.00 & 40.00 & 23500.00 & 4.57266536\end{array}$




\begin{tabular}{|c|c|c|c|}
\hline 7500.00 & 50.00 & 23500.00 & 5.58856719 \\
\hline 9000.00 & 60.00 & 23500.00 & 6.76060423 \\
\hline 8000.00 & 70.00 & 23500.00 & 7.55303595 \\
\hline 9000.00 & 130.00 & 23500.00 & 12.72957221 \\
\hline 8500.00 & 140.00 & 23500.00 & 13.17570879 \\
\hline 9000.00 & 190.00 & 23500.00 & 16.55580066 \\
\hline 5000.00 & 10.00 & 26000.00 & 1.09700851 \\
\hline 3000.00 & 20.00 & 26000.00 & 1.91099061 \\
\hline 6000.00 & 30.00 & 26000.00 & 3.09763258 \\
\hline 6500.00 & 40.00 & 26000.00 & 4.05515046 \\
\hline 6500.00 & 50.00 & 26000.00 & 4.92922075 \\
\hline 8000.00 & 60.00 & 26000.00 & 6.00229750 \\
\hline 8000.00 & 100.00 & 26000.00 & 9.13238262 \\
\hline 5000.00 & 10.00 & 28500.00 & 0.99964411 \\
\hline 6000.00 & 20.00 & 28500.00 & 1.95687938 \\
\hline 4500.00 & 30.00 & 28500.00 & 2.69621316 \\
\hline 6000.00 & 40.00 & 28500.00 & 3.65627953 \\
\hline 6500.00 & 50.00 & 28500.00 & 4.50023844 \\
\hline 8000.00 & 60.00 & 28500.00 & 5.47378290 \\
\hline 7500.00 & 80.00 & 28500.00 & 6.88067491 \\
\hline 8500.00 & 90.00 & 28500.00 & 7.80733896 \\
\hline 9000.00 & 120.00 & 28500.00 & 9.94796644 \\
\hline
\end{tabular}

The following is the list of transfer times for the maximum optimum payload delivered per day.

$\begin{array}{crcc}\mathrm{m} 0(\mathrm{~kg}) & \mathrm{PO}(\mathrm{kW}) & \text { ISP }(\mathrm{sec}) & \text { TOF (days) } \\ & & & \\ 9000.00 & 130.00 & 1000.00 & 53.5552 \\ 8000.00 & 80.00 & 3500.00 & 124.9487 \\ 9000.00 & 170.00 & 6000.00 & 110.6547 \\ 9000.00 & 80.00 & 8500.00 & 304.8503\end{array}$




$\begin{array}{llll}8500.00 & 150.00 & 11000.00 & 202.1461 \\ 9000.00 & 100.00 & 13500.00 & 392.2133 \\ 9000.00 & 130.00 & 16000.00 & 357.7031 \\ 8500.00 & 140.00 & 18500.00 & 363.7434 \\ 8500.00 & 160.00 & 21000.00 & 361.5730 \\ 9000.00 & 190.00 & 23500.00 & 361.2277 \\ 8000.00 & 100.00 & 26000.00 & 672.6456 \\ 9000.00 & 120.00 & 28500.00 & 691.5612\end{array}$




\section{Appendix B}

\section{Appendix: Near Term Plots}

Figures B.1 through B.2 present the initial mass versus payload mass in low lunar orbit for nine power levels, $10 \mathrm{~kW}, 12.5 \mathrm{~kW}, 15 \mathrm{~kW}, 17.5 \mathrm{~kW}, 20 \mathrm{~kW}, 22.5 \mathrm{~kW}$, $25 \mathrm{~kW}, 27.5 \mathrm{~kW}$, and $30 \mathrm{~kW}$.

Figures B.3 through B.4 present the payload mass per day as a function of initial mass.

Figures B.5 to B.6 highlight the transfer times for a range of specific impulses, from $1250 \mathrm{sec}$ to $3000 \mathrm{sec}$. 

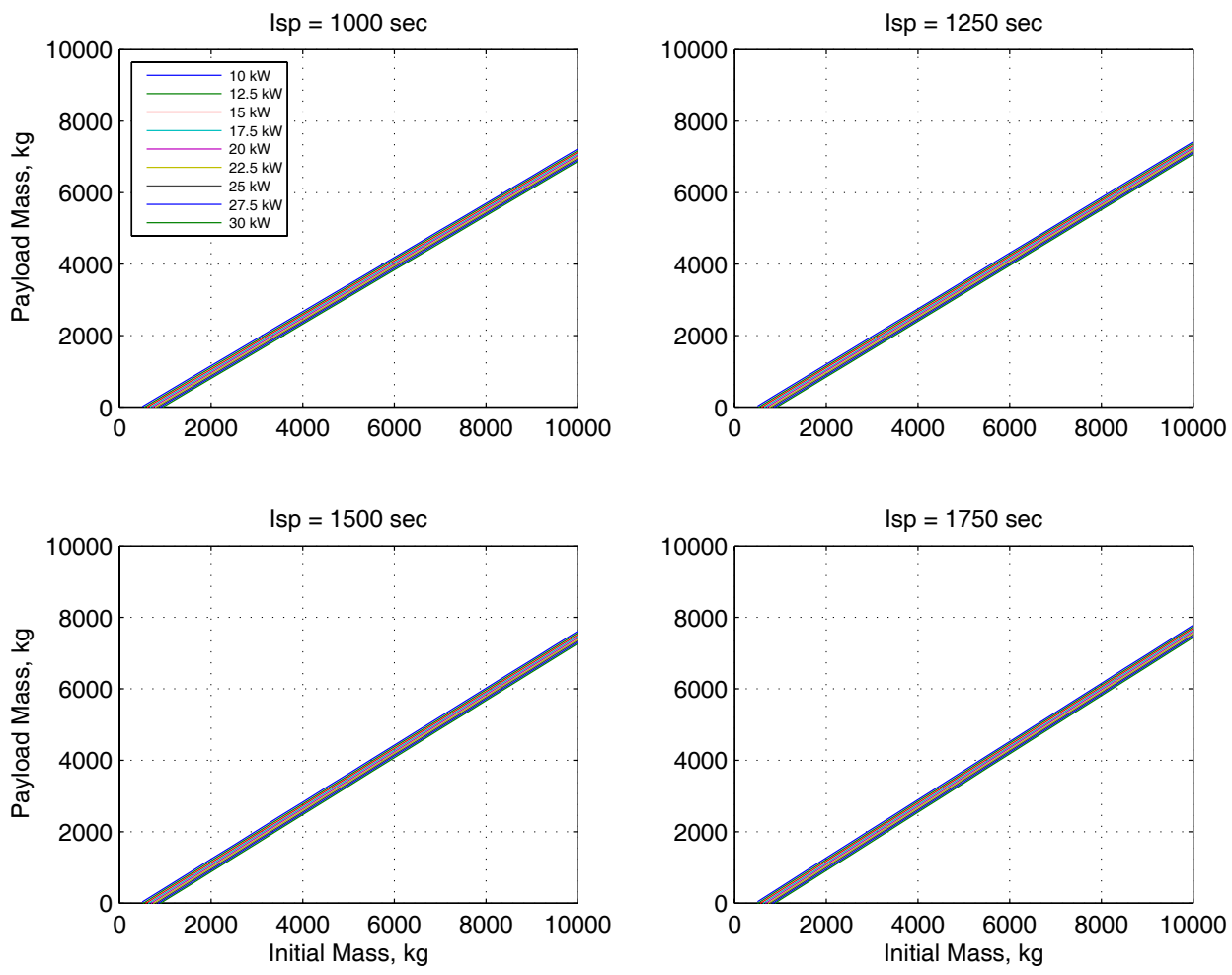

Figure B.1: Initial Mass versus Payload Mass 

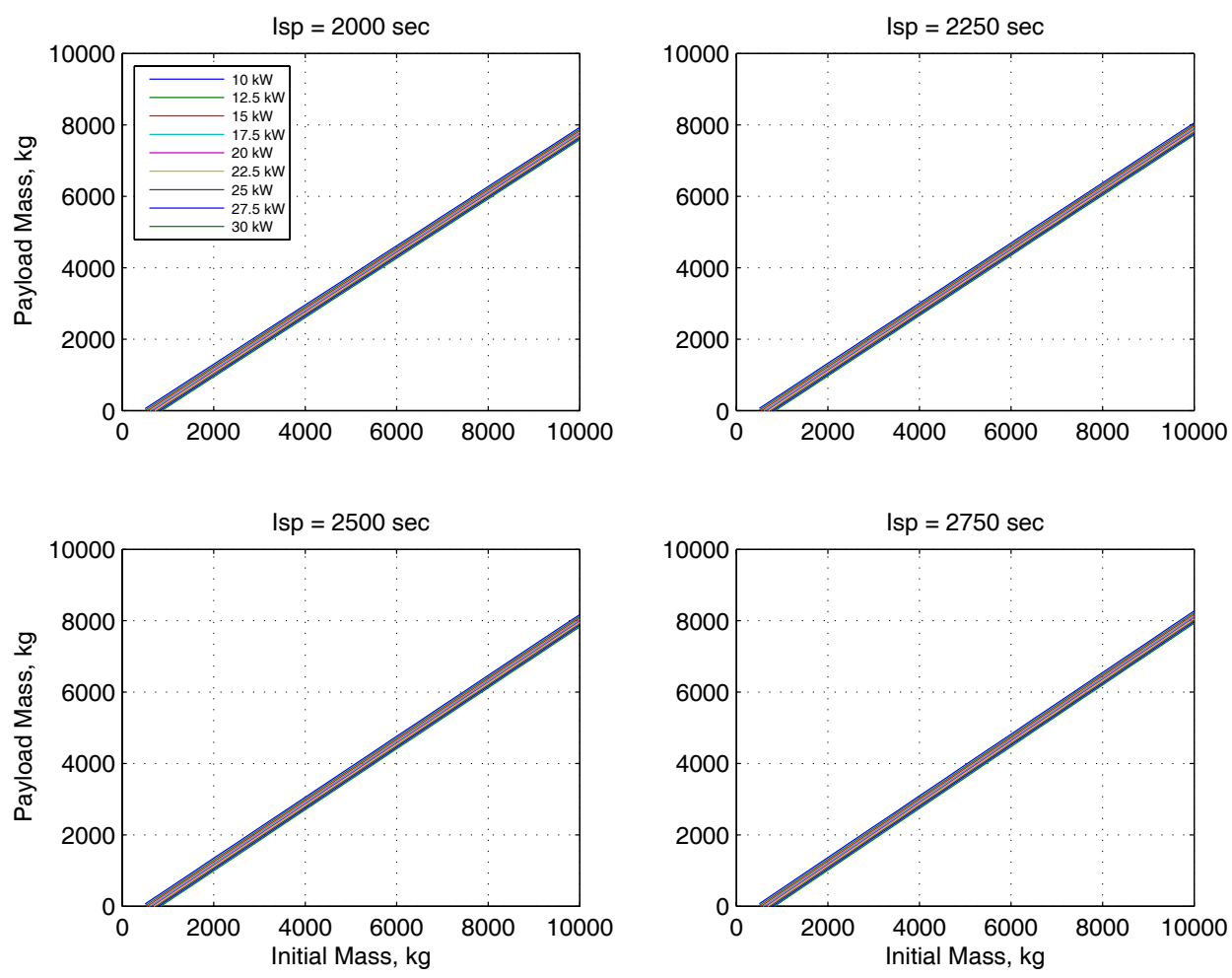

Figure B.2: Initial Mass versus Payload Mass 

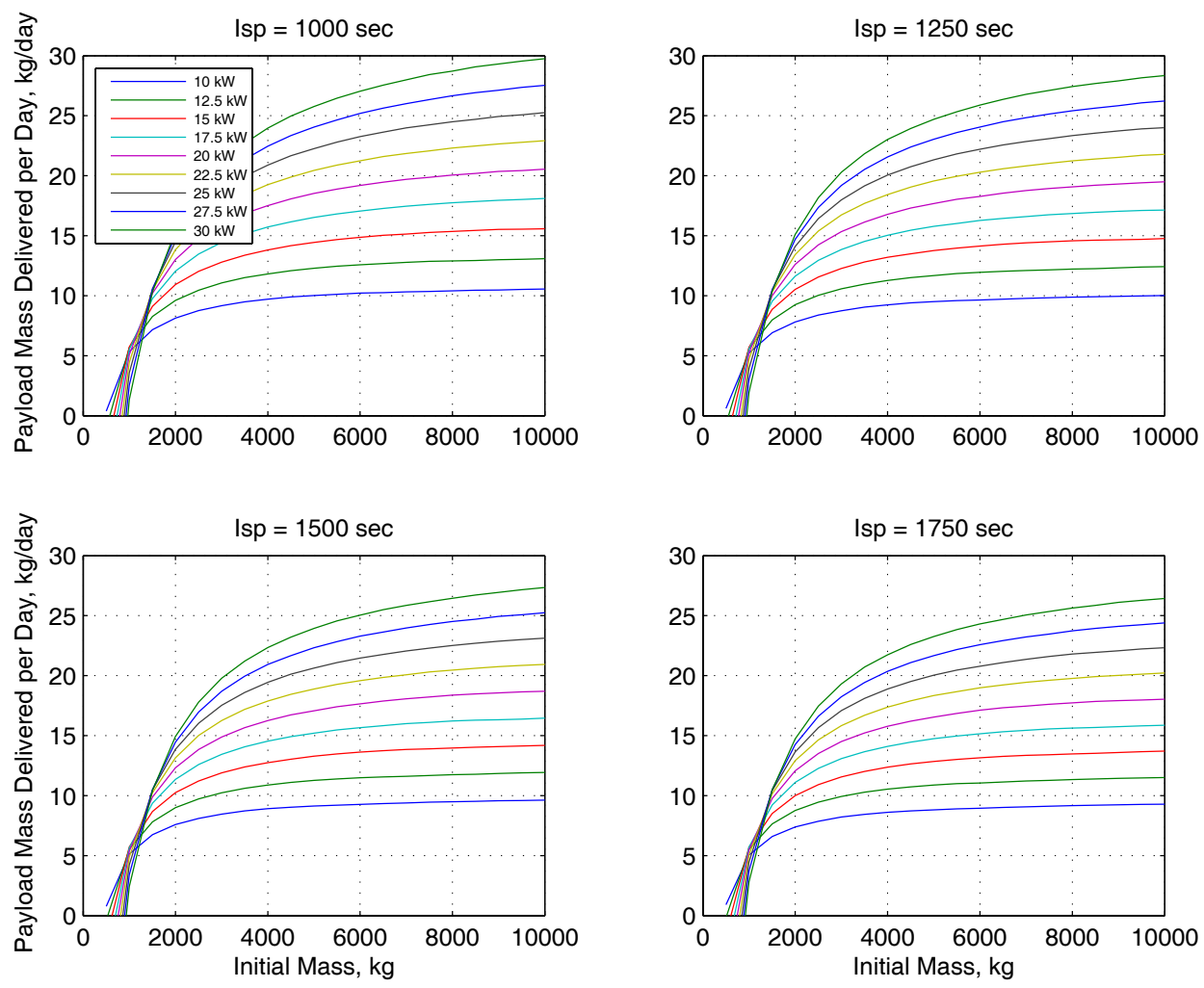

Figure B.3: Initial Mass vs. Payload Mass per Day 

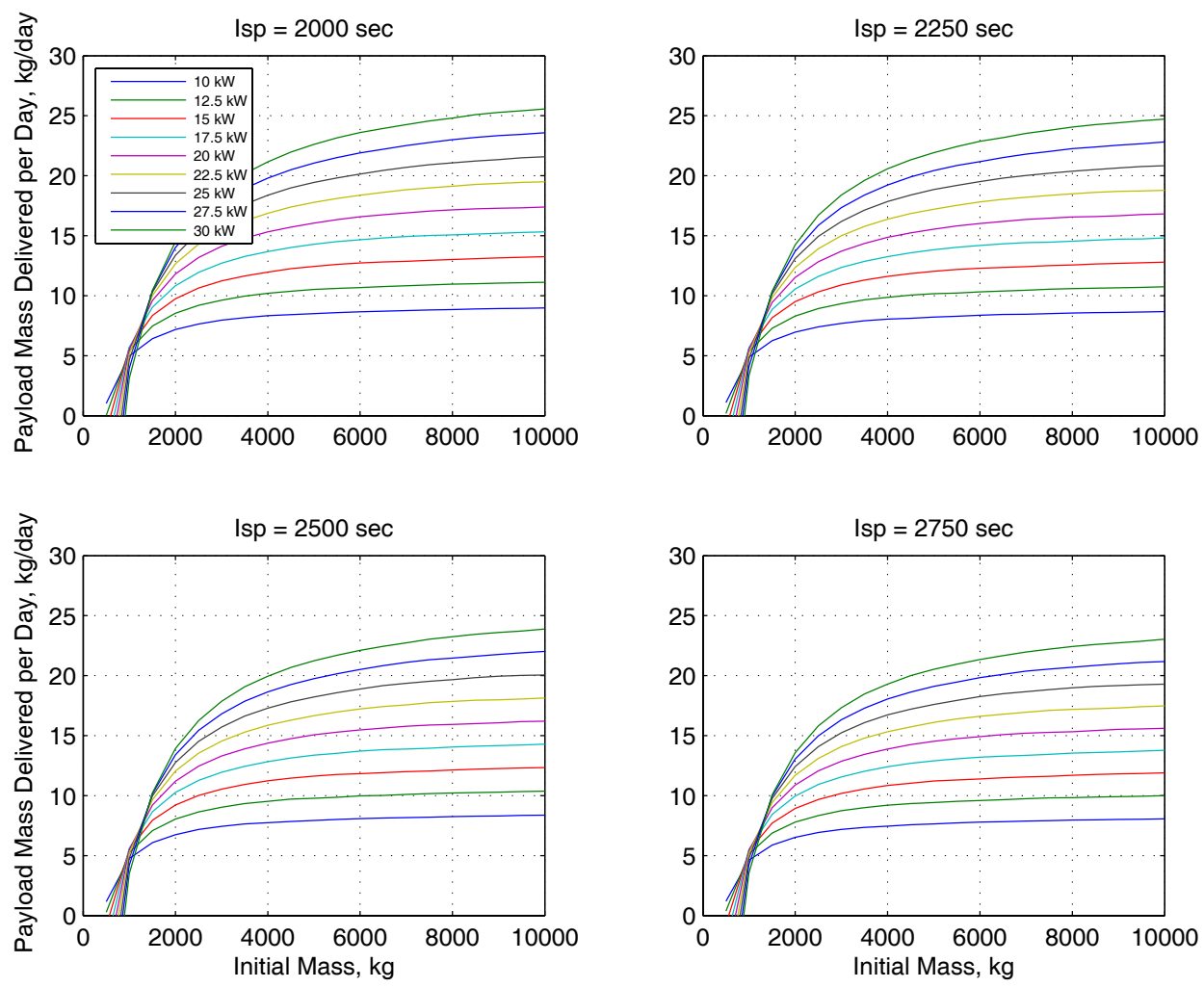

Figure B.4: Initial Mass vs. Payload Mass per Day 

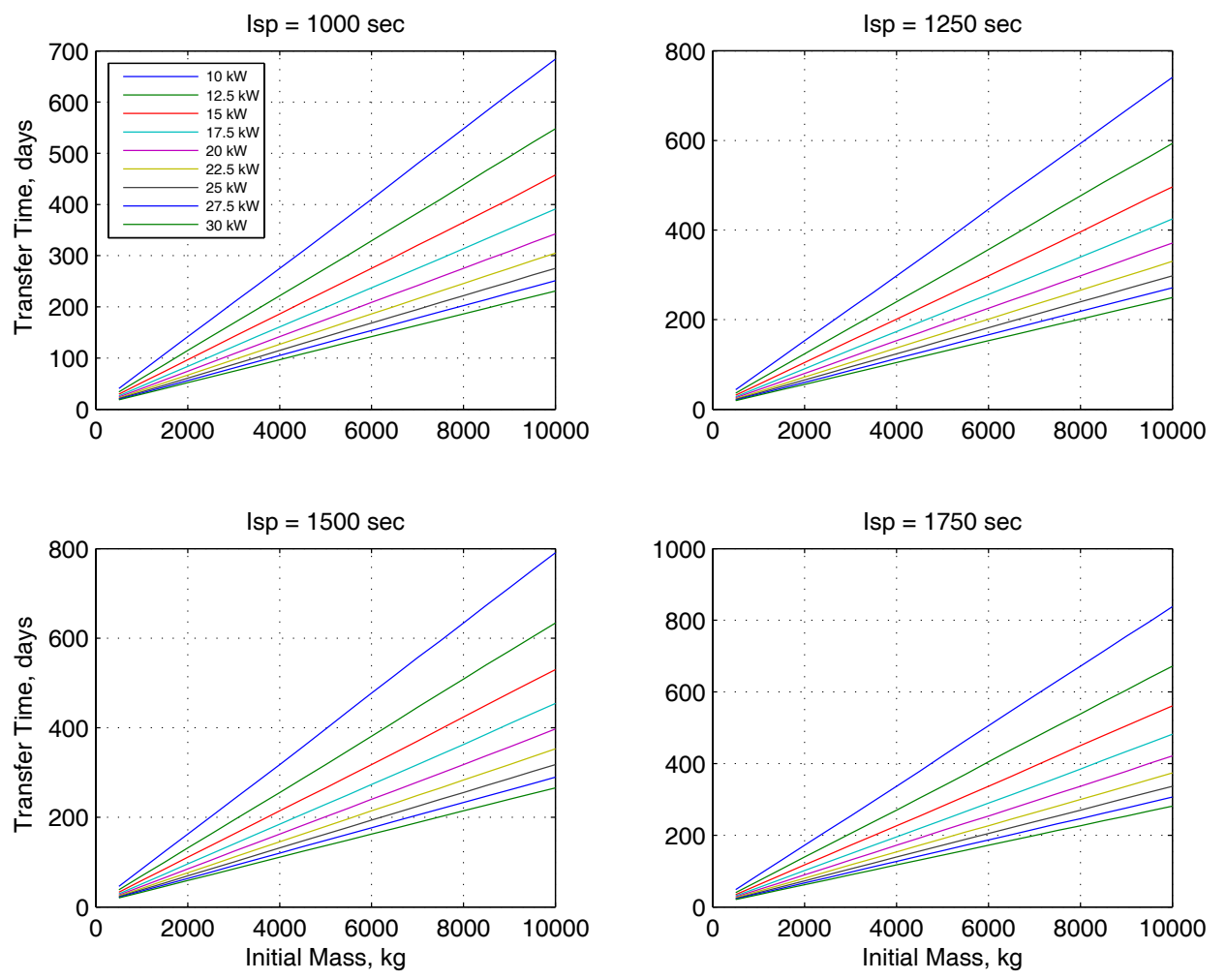

Figure B.5: Initial Mass vs. Payload Mass per Day 

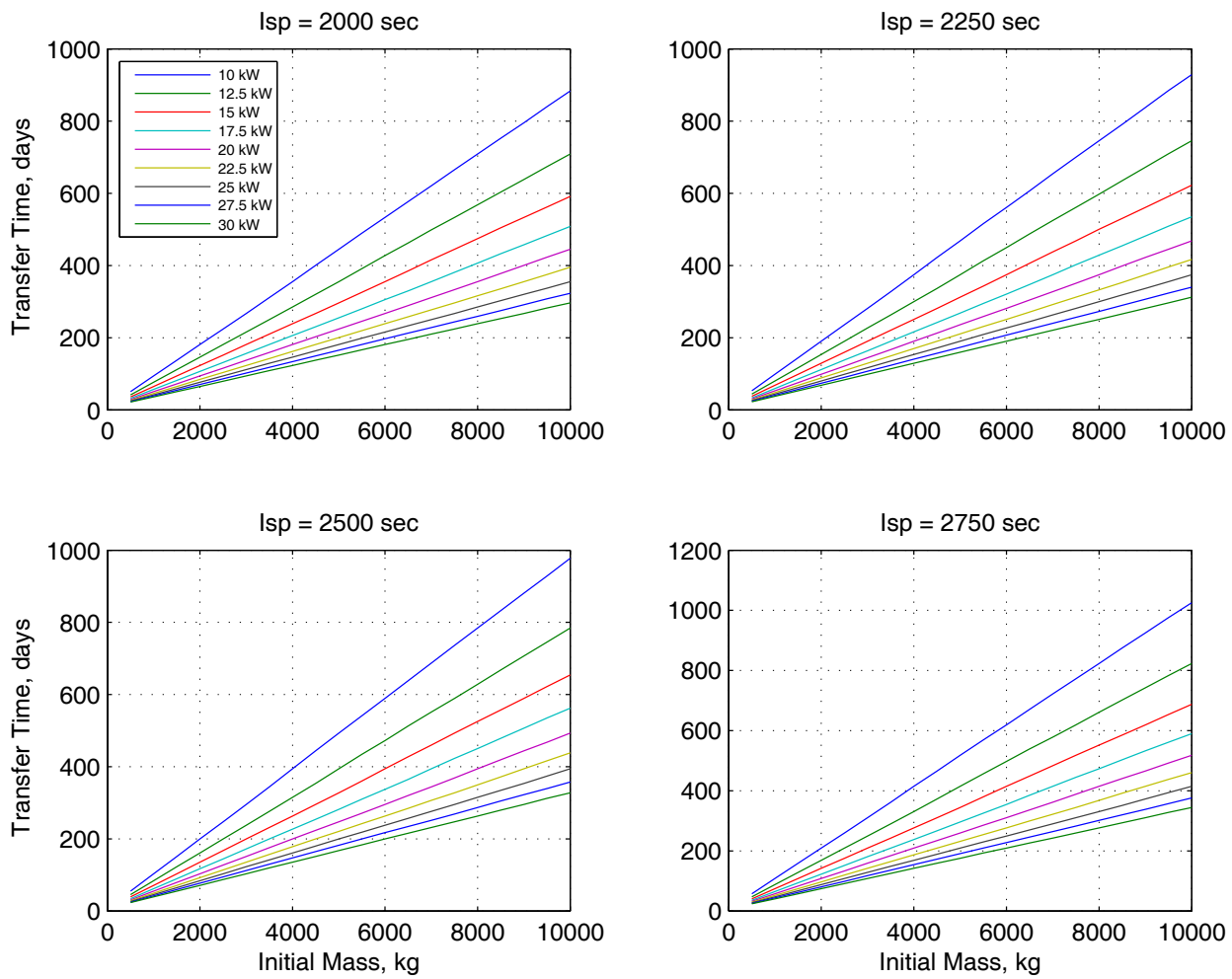

Figure B.6: Initial Mass vs. Payload Mass per Day 
The following are the tabulated results for the points of diminishing returns, where one exists for a combination of initial mass, power, and specific impulse, sorted by power. There are 77 cases that have a point of diminishing returns for the initial mass versus payload delivered per day curves.

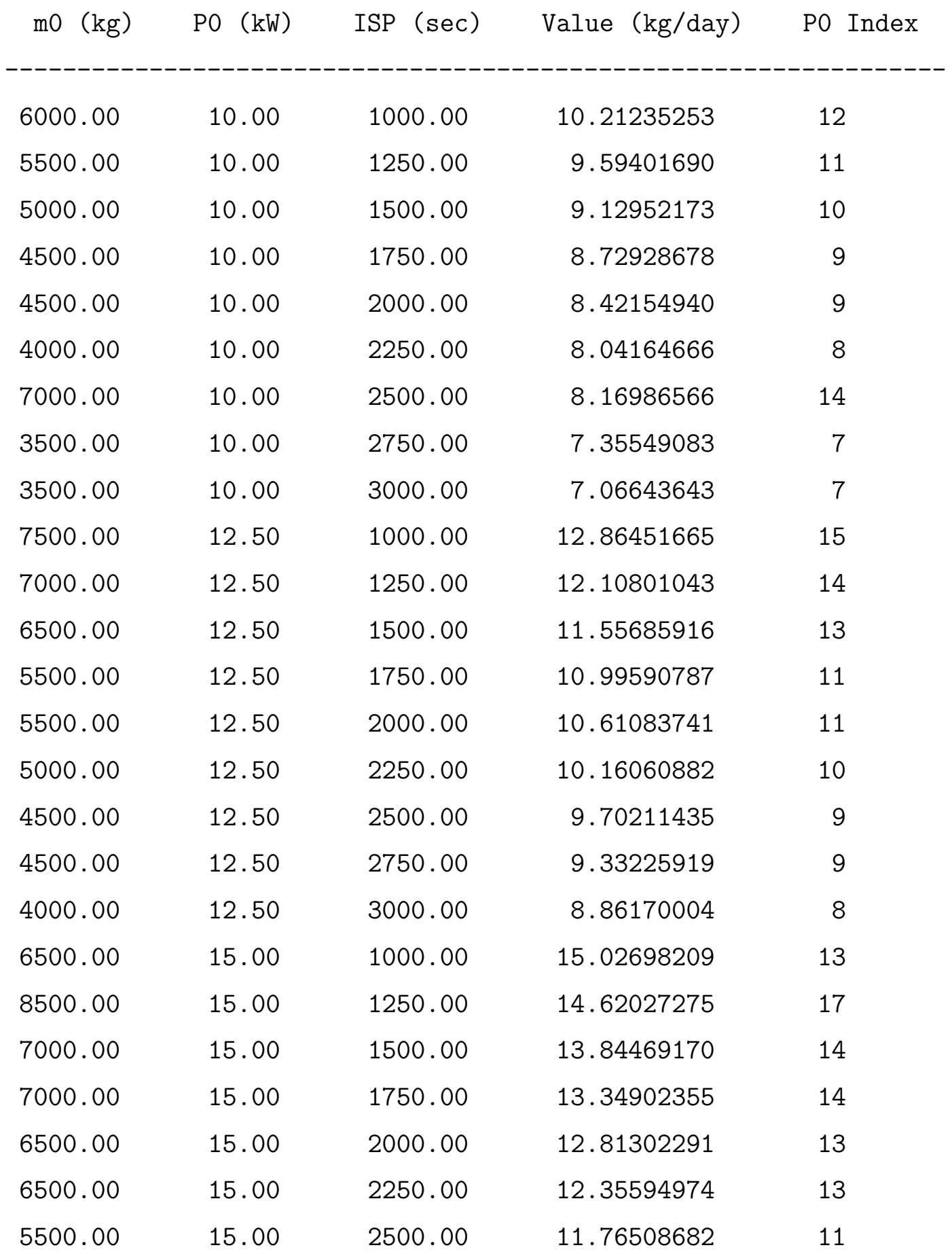




\begin{tabular}{|c|c|c|c|}
\hline 5500.00 & 15.00 & 2750.00 & 11.30755788 \\
\hline 5000.00 & 15.00 & 3000.00 & 10.77890549 \\
\hline 8000.00 & 17.50 & 1000.00 & 17.74286817 \\
\hline 5000.00 & 17.50 & 1250.00 & 15.77973345 \\
\hline 7000.00 & 17.50 & 1500.00 & 15.99728090 \\
\hline 8000.00 & 17.50 & 1750.00 & 15.62959572 \\
\hline 5500.00 & 17.50 & 2000.00 & 14.50227610 \\
\hline 7000.00 & 17.50 & 2250.00 & 14.41728188 \\
\hline 5000.00 & 17.50 & 2500.00 & 13.37432884 \\
\hline 6000.00 & 17.50 & 2750.00 & 13.20806585 \\
\hline 4500.00 & 17.50 & 3000.00 & 12.21163103 \\
\hline 7000.00 & 20.00 & 1000.00 & 19.69492524 \\
\hline 5500.00 & 20.00 & 1250.00 & 18.02940501 \\
\hline 9000.00 & 20.00 & 1750.00 & 17.92209335 \\
\hline 6500.00 & 20.00 & 2000.00 & 16.75251896 \\
\hline 8000.00 & 20.00 & 2250.00 & 16.55228278 \\
\hline 7500.00 & 20.00 & 2500.00 & 15.87962448 \\
\hline 7500.00 & 20.00 & 2750.00 & 15.26790016 \\
\hline 7000.00 & 20.00 & 3000.00 & 14.59359132 \\
\hline 7000.00 & 22.50 & 1000.00 & 21.85896635 \\
\hline 8500.00 & 22.50 & 1250.00 & 21.38684277 \\
\hline 6500.00 & 22.50 & 1500.00 & 19.85269093 \\
\hline 5000.00 & 22.50 & 1750.00 & 18.33393219 \\
\hline 7500.00 & 22.50 & 2000.00 & 18.98770242 \\
\hline 6500.00 & 22.50 & 2250.00 & 18.04281248 \\
\hline 6500.00 & 22.50 & 2500.00 & 17.42184127 \\
\hline 8000.00 & 22.50 & 2750.00 & 17.19384470 \\
\hline 6000.00 & 22.50 & 3000.00 & 15.98989642 \\
\hline 8000.00 & 25.00 & 1000.00 & 24.49106179 \\
\hline 7000.00 & 25.00 & 1250.00 & 22.84678181 \\
\hline 7000.00 & 25.00 & 1500.00 & 22.06217911 \\
\hline
\end{tabular}




\begin{tabular}{|c|c|c|c|}
\hline 8500.00 & 25.00 & 1750.00 & 21.94072755 \\
\hline 8500.00 & 25.00 & 2000.00 & 21.21639333 \\
\hline 7500.00 & 25.00 & 2500.00 & 19.53190563 \\
\hline 6500.00 & 25.00 & 2750.00 & 18.49452547 \\
\hline 7000.00 & 25.00 & 3000.00 & 17.96104813 \\
\hline 8500.00 & 27.50 & 1000.00 & 26.92916707 \\
\hline 8500.00 & 27.50 & 1250.00 & 25.63847602 \\
\hline 6000.00 & 27.50 & 1500.00 & 23.29496981 \\
\hline 7000.00 & 27.50 & 1750.00 & 23.22170620 \\
\hline 7000.00 & 27.50 & 2000.00 & 22.52039347 \\
\hline 5500.00 & 27.50 & 2250.00 & 20.85258664 \\
\hline 7500.00 & 27.50 & 2500.00 & 21.31970028 \\
\hline 7000.00 & 27.50 & 3000.00 & 19.60115211 \\
\hline 7500.00 & 30.00 & 1000.00 & 28.42505854 \\
\hline 7000.00 & 30.00 & 1250.00 & 26.78118425 \\
\hline 8500.00 & 30.00 & 1500.00 & 26.71277924 \\
\hline 7000.00 & 30.00 & 1750.00 & 25.06166696 \\
\hline 6500.00 & 30.00 & 2000.00 & 23.94433851 \\
\hline 6000.00 & 30.00 & 2250.00 & 22.86041554 \\
\hline 6500.00 & 30.00 & 2500.00 & 22.43872314 \\
\hline 9000.00 & 30.00 & 2750.00 & 22.73798042 \\
\hline
\end{tabular}

The following are the tabulated results for the points of diminishing returns, where one exists for a combination of initial mass, power, and specific impulse, sorted by specific impulse.

$\begin{array}{llccc}\mathrm{m} 0(\mathrm{~kg}) & \text { P0 }(\mathrm{kW}) & \text { ISP }(\mathrm{sec}) & \text { Value }(\mathrm{kg} / \text { day }) & \text { P0 Index } \\ & & & \\ 6000.00 & 10.00 & 1000.00 & 10.21235253 & 12 \\ 7500.00 & 12.50 & 1000.00 & 12.86451665 & 15 \\ 6500.00 & 15.00 & 1000.00 & 15.02698209 & 13 \\ 8000.00 & 17.50 & 1000.00 & 17.74286817 & 16\end{array}$




\begin{tabular}{|c|c|c|c|}
\hline 7000.00 & 20.00 & 1000.00 & 19.69492524 \\
\hline 7000.00 & 22.50 & 1000.00 & 21.85896635 \\
\hline 8000.00 & 25.00 & 1000.00 & 24.49106179 \\
\hline 8500.00 & 27.50 & 1000.00 & 26.92916707 \\
\hline 7500.00 & 30.00 & 1000.00 & 28.42505854 \\
\hline 5500.00 & 10.00 & 1250.00 & 9.59401690 \\
\hline 7000.00 & 12.50 & 1250.00 & 12.10801043 \\
\hline 8500.00 & 15.00 & 1250.00 & 14.62027275 \\
\hline 5000.00 & 17.50 & 1250.00 & 15.77973345 \\
\hline 5500.00 & 20.00 & 1250.00 & 18.02940501 \\
\hline 8500.00 & 22.50 & 1250.00 & 21.38684277 \\
\hline 7000.00 & 25.00 & 1250.00 & 22.84678181 \\
\hline 8500.00 & 27.50 & 1250.00 & 25.63847602 \\
\hline 7000.00 & 30.00 & 1250.00 & 26.78118425 \\
\hline 5000.00 & 10.00 & 1500.00 & 9.12952173 \\
\hline 6500.00 & 12.50 & 1500.00 & 11.55685916 \\
\hline 7000.00 & 15.00 & 1500.00 & 13.84469170 \\
\hline 7000.00 & 17.50 & 1500.00 & 15.99728090 \\
\hline 6500.00 & 22.50 & 1500.00 & 19.85269093 \\
\hline 7000.00 & 25.00 & 1500.00 & 22.06217911 \\
\hline 6000.00 & 27.50 & 1500.00 & 23.29496981 \\
\hline 8500.00 & 30.00 & 1500.00 & 26.71277924 \\
\hline 4500.00 & 10.00 & 1750.00 & 8.72928678 \\
\hline 5500.00 & 12.50 & 1750.00 & 10.99590787 \\
\hline 7000.00 & 15.00 & 1750.00 & 13.34902355 \\
\hline 8000.00 & 17.50 & 1750.00 & 15.62959572 \\
\hline 9000.00 & 20.00 & 1750.00 & 17.92209335 \\
\hline 5000.00 & 22.50 & 1750.00 & 18.33393219 \\
\hline 8500.00 & 25.00 & 1750.00 & 21.94072755 \\
\hline 7000.00 & 27.50 & 1750.00 & 23.22170620 \\
\hline 7000.00 & 30.00 & 1750.00 & 25.06166696 \\
\hline
\end{tabular}




\begin{tabular}{|c|c|c|c|}
\hline 4500.00 & 10.00 & 2000.00 & 8.42154940 \\
\hline 5500.00 & 12.50 & 2000.00 & 10.61083741 \\
\hline 6500.00 & 15.00 & 2000.00 & 12.81302291 \\
\hline 5500.00 & 17.50 & 2000.00 & 14.50227610 \\
\hline 6500.00 & 20.00 & 2000.00 & 16.75251896 \\
\hline 7500.00 & 22.50 & 2000.00 & 18.98770242 \\
\hline 8500.00 & 25.00 & 2000.00 & 21.21639333 \\
\hline 7000.00 & 27.50 & 2000.00 & 22.52039347 \\
\hline 6500.00 & 30.00 & 2000.00 & 23.94433851 \\
\hline 4000.00 & 10.00 & 2250.00 & 8.04164666 \\
\hline 5000.00 & 12.50 & 2250.00 & 10.16060882 \\
\hline 6500.00 & 15.00 & 2250.00 & 12.35594974 \\
\hline 7000.00 & 17.50 & 2250.00 & 14.41728188 \\
\hline 8000.00 & 20.00 & 2250.00 & 16.55228278 \\
\hline 6500.00 & 22.50 & 2250.00 & 18.04281248 \\
\hline 5500.00 & 27.50 & 2250.00 & 20.85258664 \\
\hline 6000.00 & 30.00 & 2250.00 & 22.86041554 \\
\hline 7000.00 & 10.00 & 2500.00 & 8.16986566 \\
\hline 4500.00 & 12.50 & 2500.00 & 9.70211435 \\
\hline 5500.00 & 15.00 & 2500.00 & 11.76508682 \\
\hline 5000.00 & 17.50 & 2500.00 & 13.37432884 \\
\hline 7500.00 & 20.00 & 2500.00 & 15.87962448 \\
\hline 6500.00 & 22.50 & 2500.00 & 17.42184127 \\
\hline 7500.00 & 25.00 & 2500.00 & 19.53190563 \\
\hline 7500.00 & 27.50 & 2500.00 & 21.31970028 \\
\hline 6500.00 & 30.00 & 2500.00 & 22.43872314 \\
\hline 3500.00 & 10.00 & 2750.00 & 7.35549083 \\
\hline 4500.00 & 12.50 & 2750.00 & 9.33225919 \\
\hline 5500.00 & 15.00 & 2750.00 & 11.30755788 \\
\hline 6000.00 & 17.50 & 2750.00 & 13.20806585 \\
\hline 7500.00 & 20.00 & 2750.00 & 15.26790016 \\
\hline
\end{tabular}




$\begin{array}{rrrrr}8000.00 & 22.50 & 2750.00 & 17.19384470 & 16 \\ 6500.00 & 25.00 & 2750.00 & 18.49452547 & 13 \\ 9000.00 & 30.00 & 2750.00 & 22.73798042 & 18 \\ 3500.00 & 10.00 & 3000.00 & 7.06643643 & 7 \\ 4000.00 & 12.50 & 3000.00 & 8.86170004 & 8 \\ 5000.00 & 15.00 & 3000.00 & 10.77890549 & 10 \\ 4500.00 & 17.50 & 3000.00 & 12.21163103 & 9 \\ 7000.00 & 20.00 & 3000.00 & 14.59359132 & 14 \\ 6000.00 & 22.50 & 3000.00 & 15.98989642 & 12 \\ 7000.00 & 25.00 & 3000.00 & 17.96104813 & 14 \\ 7000.00 & 27.50 & 3000.00 & 19.60115211 & 14\end{array}$

The following is the list of transfer times for the maximum optimum payload delivered per day.

The following is the list of transfer times for the maximum optimum payload per day $\mathrm{mo}(\mathrm{kg}) \quad \mathrm{PO}(\mathrm{kW}) \quad$ ISP (sec) TOF (days)

$\begin{array}{llll}7500.00 & 30.00 & 1000.00 & 175.0357 \\ 7000.00 & 30.00 & 1250.00 & 176.9327 \\ 8500.00 & 30.00 & 1500.00 & 227.2332 \\ 7000.00 & 30.00 & 1750.00 & 199.2531 \\ 6500.00 & 30.00 & 2000.00 & 195.5445 \\ 6000.00 & 30.00 & 2250.00 & 190.0674 \\ 6500.00 & 30.00 & 2500.00 & 215.6425 \\ 9000.00 & 30.00 & 2750.00 & 310.6153 \\ 7000.00 & 27.50 & 3000.00 & 277.3062\end{array}$




\section{Appendix $\mathrm{C}$}

\section{Appendix: Mid Term Plots}

The following plots, Figures C.1 to C.2, present the initial mass versus payload mass in low lunar orbit for fifteen power levels $30 \mathrm{~kW}$ to $100 \mathrm{~kW}$, by increments of $5 \mathrm{~kW}$.
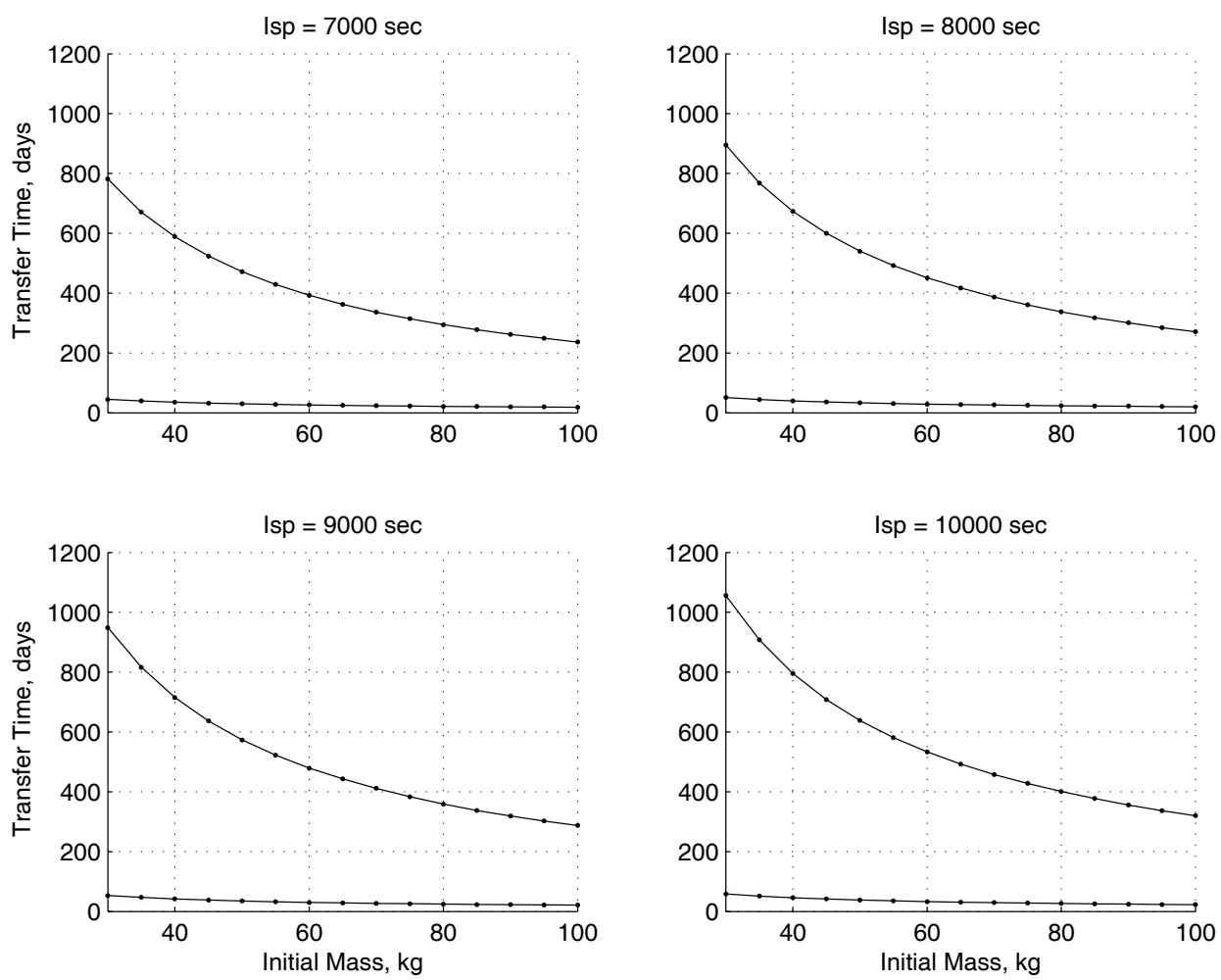

Figure C.1: Power vs. Total Time of Flight 

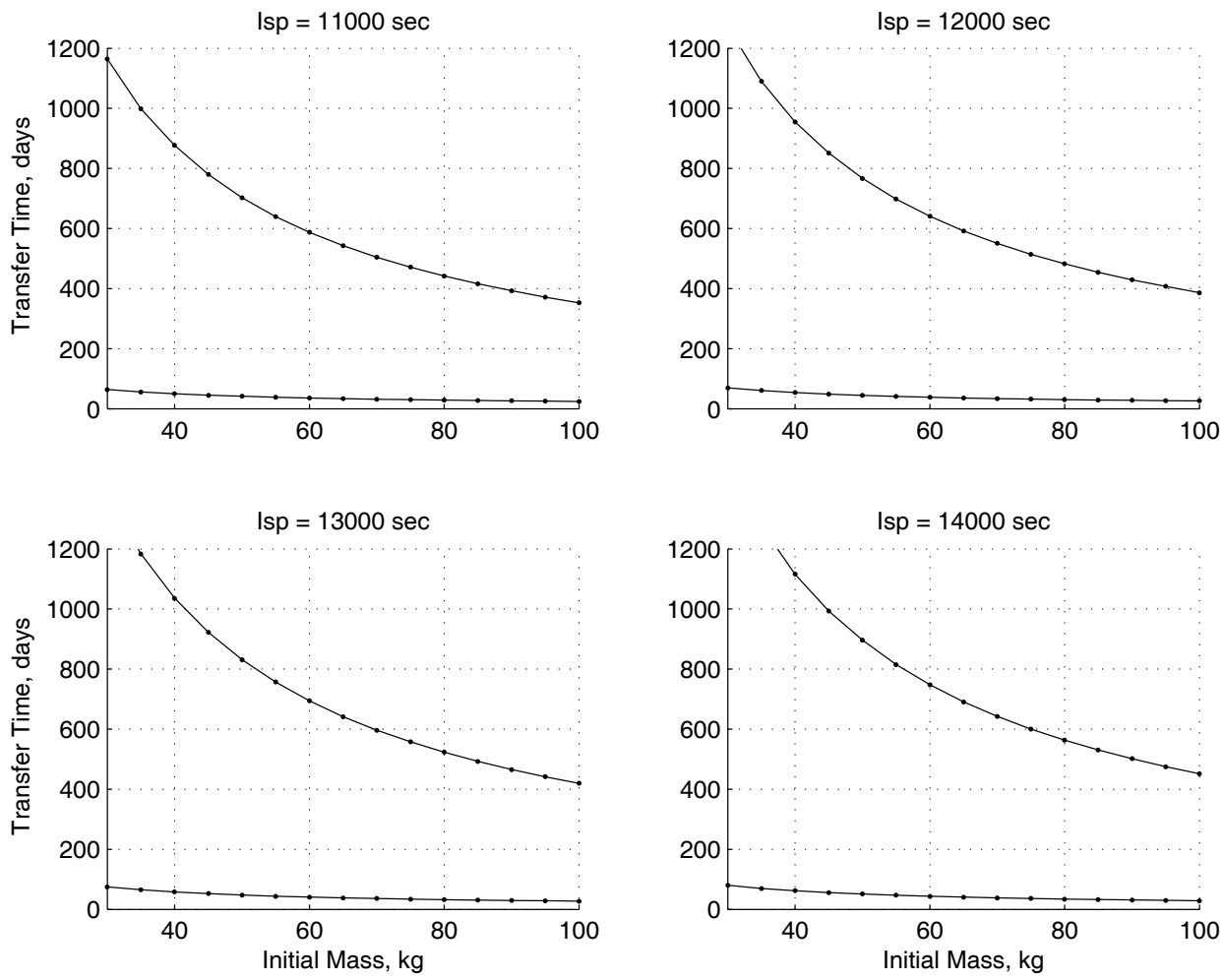

Figure C.2: Power vs. Total Time of Flight 
The following plots, Figures C.3 to C.4, present the transfer time as a function of initial mass for the mid term technology levels.
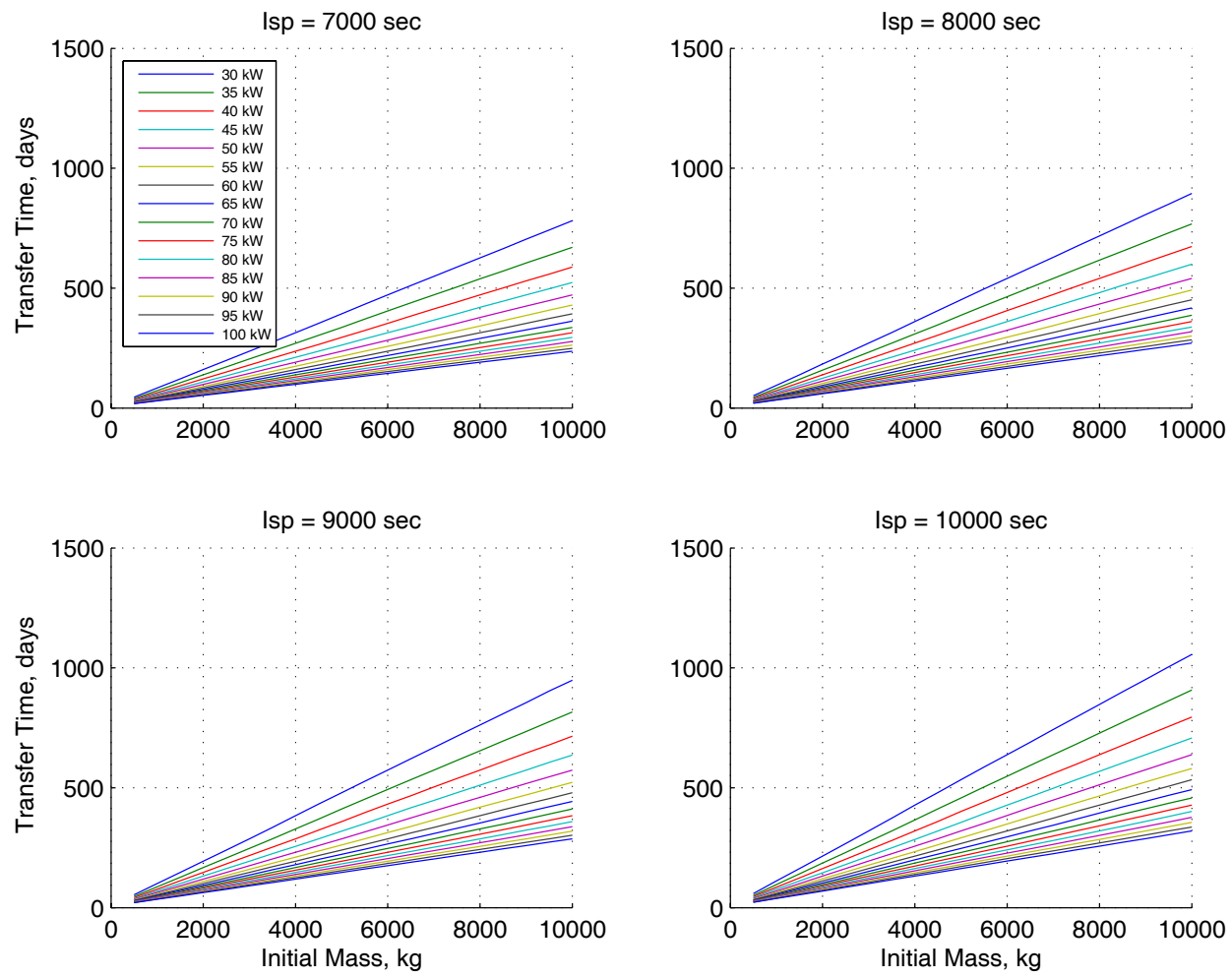

Figure C.3: Initial Mass vs. Total Time of Flight 

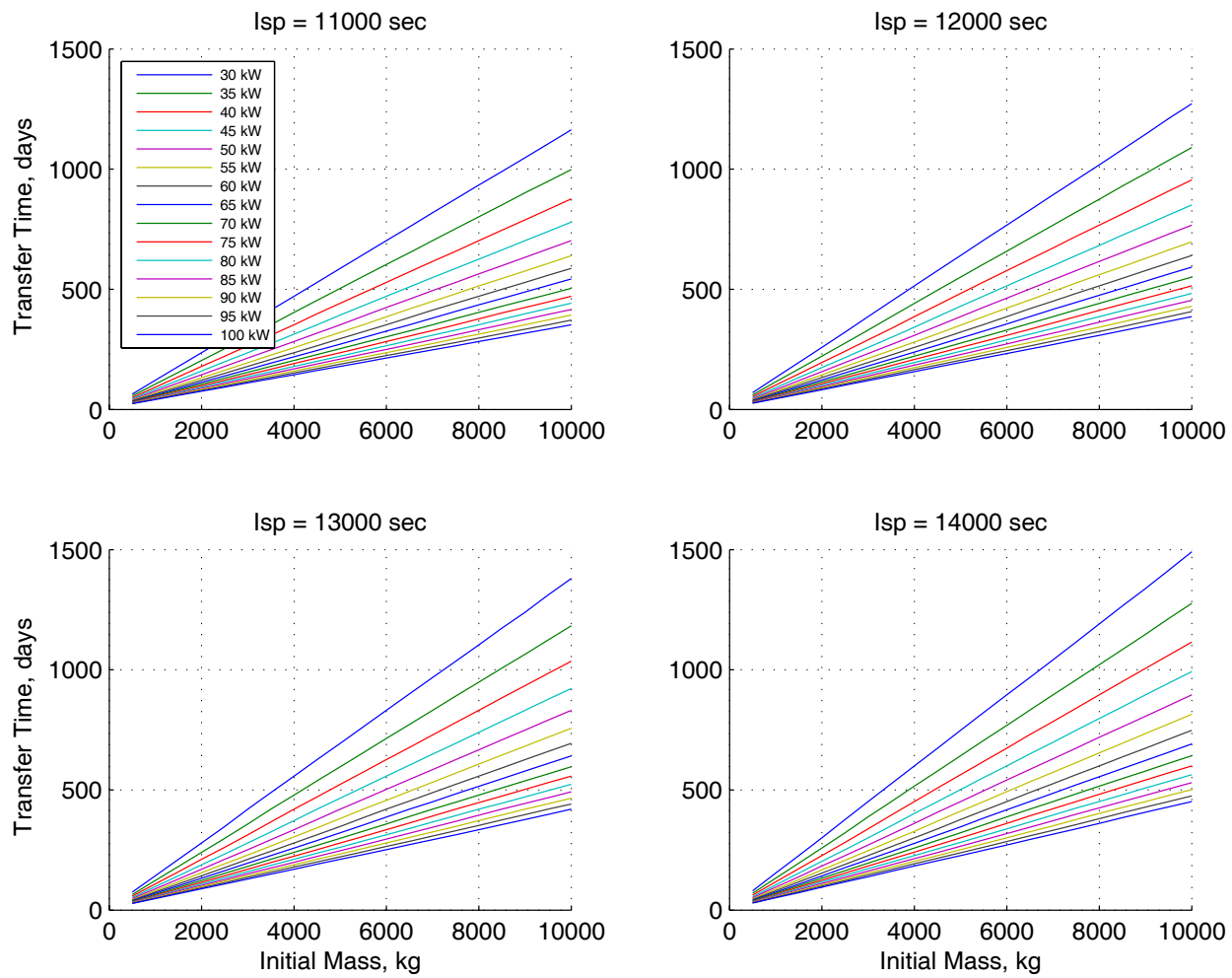

Figure C.4: Initial Mass vs. Total Time of Flight 
Figures C.5 to C.6, presents the payload mass delivered per day for the mid term technology levels.
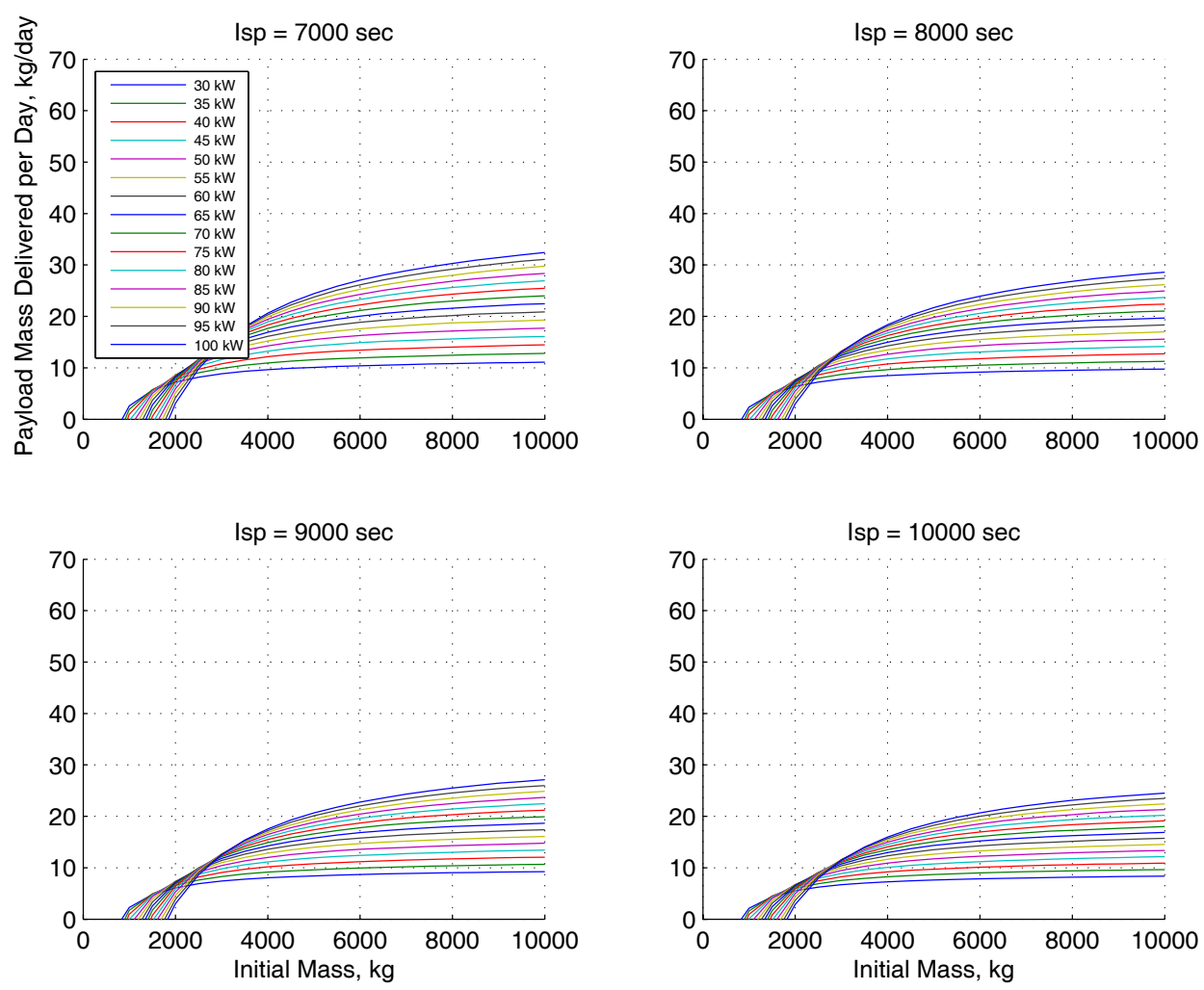

Figure C.5: Initial Mass vs. Payload Delivered Per Day 

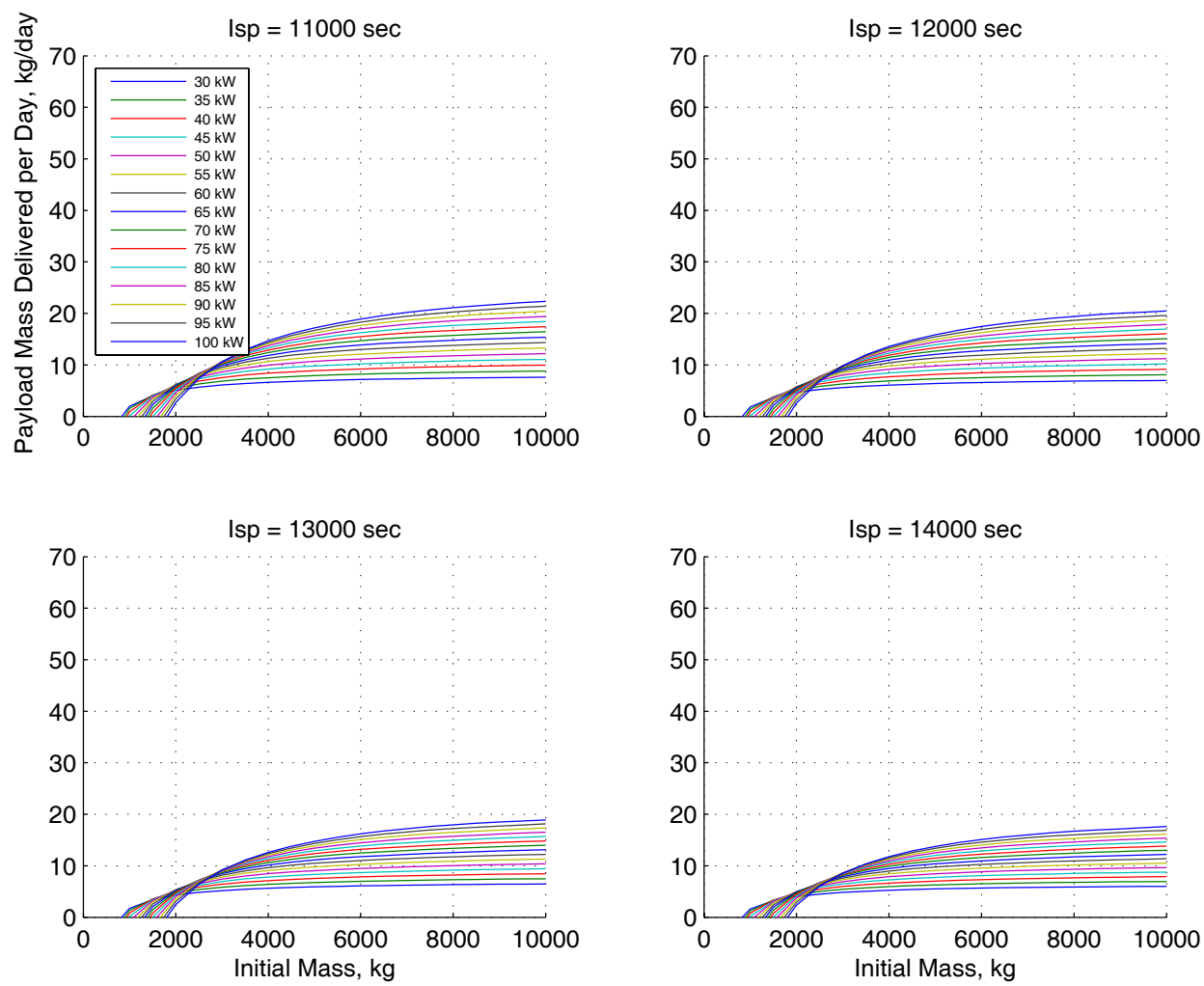

Figure C.6: Initial Mass vs. Payload Delivered Per Day 
The following are the tabulated results for the points of diminishing returns, where one exists for a combination of initial mass, power, and specific impulse, sorted by power. There are 240 cases that have a point of diminishing returns for the initial mass versus payload delivered per day curves.

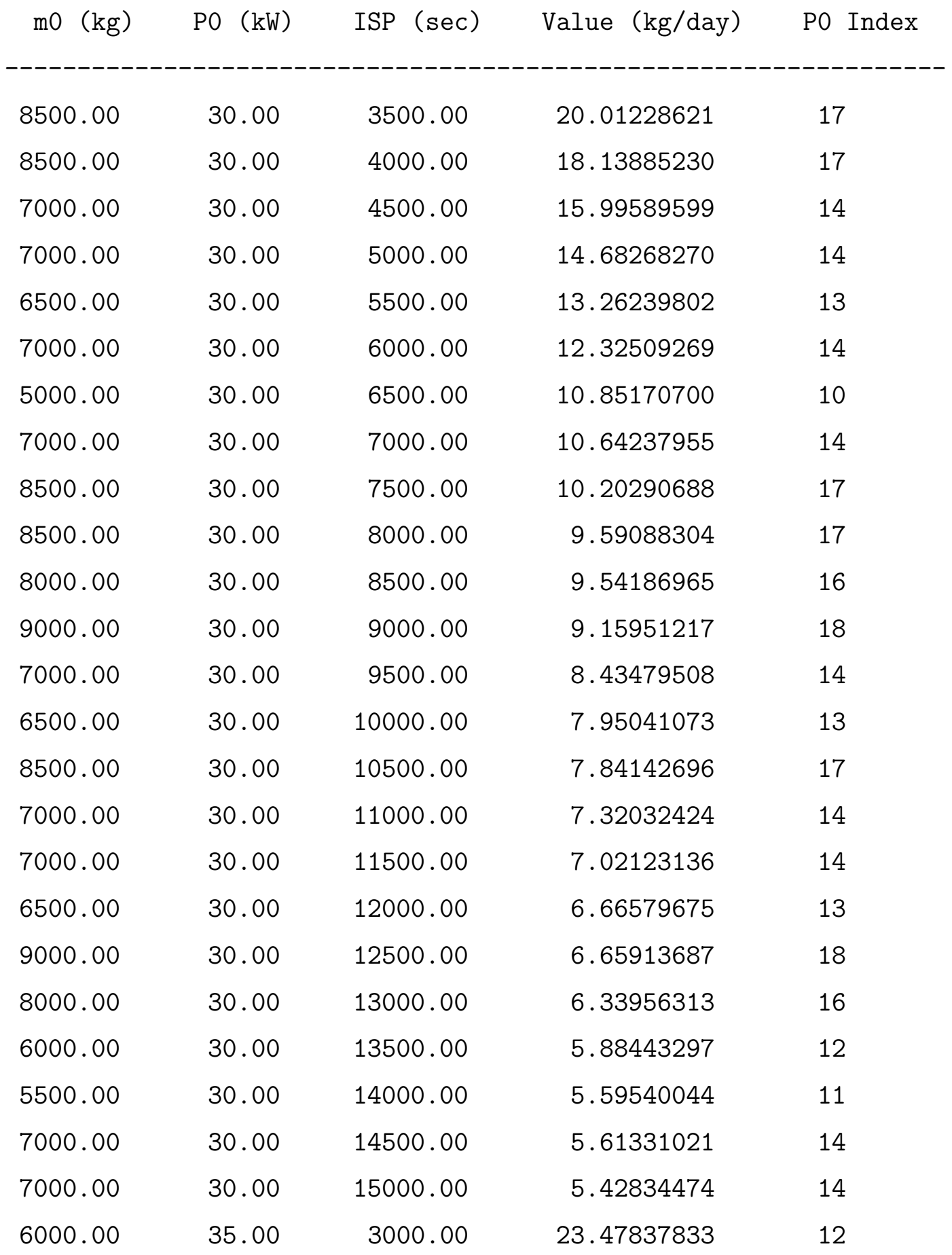




\begin{tabular}{|c|c|c|c|}
\hline 6500.00 & 35.00 & 3500.00 & 22.02911109 \\
\hline 8500.00 & 35.00 & 4500.00 & 18.83008803 \\
\hline 7500.00 & 35.00 & 5000.00 & 17.10355142 \\
\hline 7000.00 & 35.00 & 5500.00 & 15.43272916 \\
\hline 6500.00 & 35.00 & 6000.00 & 14.03768783 \\
\hline 6000.00 & 35.00 & 6500.00 & 12.82931998 \\
\hline 8500.00 & 35.00 & 7000.00 & 12.55024657 \\
\hline 8000.00 & 35.00 & 7500.00 & 11.66265043 \\
\hline 7500.00 & 35.00 & 8000.00 & 10.86850773 \\
\hline 7500.00 & 35.00 & 8500.00 & 10.88680685 \\
\hline 8000.00 & 35.00 & 9500.00 & 9.87724143 \\
\hline 6500.00 & 35.00 & 10000.00 & 9.12280962 \\
\hline 7500.00 & 35.00 & 10500.00 & 8.89061839 \\
\hline 7000.00 & 35.00 & 11000.00 & 8.41531331 \\
\hline 8000.00 & 35.00 & 11500.00 & 8.21466075 \\
\hline 7000.00 & 35.00 & 12000.00 & 7.73863014 \\
\hline 7500.00 & 35.00 & 12500.00 & 7.51557890 \\
\hline 7000.00 & 35.00 & 13000.00 & 7.16214210 \\
\hline 7000.00 & 35.00 & 13500.00 & 6.91275558 \\
\hline 6500.00 & 35.00 & 14000.00 & 6.59024356 \\
\hline 8500.00 & 35.00 & 14500.00 & 6.61142699 \\
\hline 8000.00 & 35.00 & 15000.00 & 6.35211101 \\
\hline 8000.00 & 40.00 & 3000.00 & 27.83455177 \\
\hline 7500.00 & 40.00 & 3500.00 & 25.37035181 \\
\hline 8500.00 & 40.00 & 4000.00 & 23.65065887 \\
\hline 9000.00 & 40.00 & 5000.00 & 19.73029656 \\
\hline 8500.00 & 40.00 & 5500.00 & 17.85046658 \\
\hline 7000.00 & 40.00 & 6000.00 & 16.04366841 \\
\hline 7000.00 & 40.00 & 6500.00 & 14.80290800 \\
\hline 6500.00 & 40.00 & 7000.00 & 13.61910706 \\
\hline 6000.00 & 40.00 & 7500.00 & 12.56481152 \\
\hline
\end{tabular}




\begin{tabular}{|c|c|c|c|}
\hline 5500.00 & 40.00 & 8000.00 & 11.60942127 \\
\hline 5500.00 & 40.00 & 8500.00 & 11.62875244 \\
\hline 5000.00 & 40.00 & 9000.00 & 10.79179387 \\
\hline 7500.00 & 40.00 & 10000.00 & 10.50373963 \\
\hline 7000.00 & 40.00 & 10500.00 & 9.91243620 \\
\hline 6500.00 & 40.00 & 11000.00 & 9.35797587 \\
\hline 8500.00 & 40.00 & 11500.00 & 9.34404681 \\
\hline 9000.00 & 40.00 & 12000.00 & 9.04163814 \\
\hline 7500.00 & 40.00 & 12500.00 & 8.46639503 \\
\hline 8000.00 & 40.00 & 13000.00 & 8.23023921 \\
\hline 8000.00 & 40.00 & 13500.00 & 7.94359873 \\
\hline 6000.00 & 40.00 & 14000.00 & 7.31343571 \\
\hline 7500.00 & 40.00 & 14500.00 & 7.33852750 \\
\hline 7000.00 & 40.00 & 15000.00 & 7.02977396 \\
\hline 8500.00 & 45.00 & 3500.00 & 28.75340387 \\
\hline 8500.00 & 45.00 & 4500.00 & 23.65940873 \\
\hline 7500.00 & 45.00 & 5000.00 & 21.40682874 \\
\hline 7000.00 & 45.00 & 5500.00 & 19.31383037 \\
\hline 9000.00 & 45.00 & 6000.00 & 18.40834610 \\
\hline 8000.00 & 45.00 & 6500.00 & 16.77247097 \\
\hline 6500.00 & 45.00 & 7000.00 & 15.14981005 \\
\hline 6500.00 & 45.00 & 7500.00 & 14.14664054 \\
\hline 6500.00 & 45.00 & 8000.00 & 13.26118185 \\
\hline 6500.00 & 45.00 & 8500.00 & 13.27723013 \\
\hline 6000.00 & 45.00 & 9000.00 & 12.39207382 \\
\hline 5500.00 & 45.00 & 9500.00 & 11.55410671 \\
\hline 8500.00 & 45.00 & 10000.00 & 11.87863155 \\
\hline 5000.00 & 45.00 & 10500.00 & 10.23910901 \\
\hline 7500.00 & 45.00 & 11500.00 & 10.18092125 \\
\hline 8000.00 & 45.00 & 12000.00 & 9.88731030 \\
\hline 7500.00 & 45.00 & 12500.00 & 9.40296178 \\
\hline
\end{tabular}




\begin{tabular}{|c|c|c|c|}
\hline 9000.00 & 45.00 & 13500.00 & 8.97650401 \\
\hline 7500.00 & 45.00 & 14000.00 & 8.42506566 \\
\hline 7500.00 & 45.00 & 14500.00 & 8.14228997 \\
\hline 9000.00 & 50.00 & 3000.00 & 34.46897729 \\
\hline 8000.00 & 50.00 & 3500.00 & 31.09072491 \\
\hline 7500.00 & 50.00 & 4000.00 & 28.00845546 \\
\hline 8500.00 & 50.00 & 5000.00 & 23.95450876 \\
\hline 9000.00 & 50.00 & 6000.00 & 20.29533875 \\
\hline 6500.00 & 50.00 & 6500.00 & 17.78342729 \\
\hline 7500.00 & 50.00 & 7000.00 & 17.01493166 \\
\hline 7500.00 & 50.00 & 7500.00 & 15.89134590 \\
\hline 7000.00 & 50.00 & 8000.00 & 14.74057822 \\
\hline 7000.00 & 50.00 & 8500.00 & 14.77025818 \\
\hline 6500.00 & 50.00 & 9000.00 & 13.77787066 \\
\hline 8000.00 & 50.00 & 9500.00 & 13.57301300 \\
\hline 6000.00 & 50.00 & 10000.00 & 12.22864054 \\
\hline 7500.00 & 50.00 & 10500.00 & 12.18176231 \\
\hline 6500.00 & 50.00 & 11000.00 & 11.34195358 \\
\hline 6500.00 & 50.00 & 11500.00 & 10.87009618 \\
\hline 9000.00 & 50.00 & 12000.00 & 11.04486661 \\
\hline 7500.00 & 50.00 & 12500.00 & 10.30919043 \\
\hline 7000.00 & 50.00 & 13000.00 & 9.80424891 \\
\hline 9000.00 & 50.00 & 13500.00 & 9.85899109 \\
\hline 7500.00 & 50.00 & 14000.00 & 9.24632415 \\
\hline 9000.00 & 50.00 & 14500.00 & 9.19925871 \\
\hline 7000.00 & 50.00 & 15000.00 & 8.53505507 \\
\hline 7500.00 & 55.00 & 3000.00 & 35.90675143 \\
\hline 6000.00 & 55.00 & 4000.00 & 28.62257720 \\
\hline 8500.00 & 55.00 & 4500.00 & 28.16547567 \\
\hline 8500.00 & 55.00 & 5500.00 & 23.81613038 \\
\hline 6500.00 & 55.00 & 7000.00 & 17.95020034 \\
\hline
\end{tabular}




\begin{tabular}{|c|c|c|c|}
\hline 7500.00 & 55.00 & 7500.00 & 17.31388861 \\
\hline 8000.00 & 55.00 & 8000.00 & 16.39852059 \\
\hline 8000.00 & 55.00 & 8500.00 & 16.41742772 \\
\hline 8500.00 & 55.00 & 9500.00 & 14.90969311 \\
\hline 9000.00 & 55.00 & 10000.00 & 14.30870123 \\
\hline 6000.00 & 55.00 & 11000.00 & 12.07011340 \\
\hline 5500.00 & 55.00 & 11500.00 & 11.32468077 \\
\hline 7500.00 & 55.00 & 12000.00 & 11.62998652 \\
\hline 9000.00 & 55.00 & 13000.00 & 11.12584226 \\
\hline 9000.00 & 55.00 & 13500.00 & 10.72759342 \\
\hline 8000.00 & 55.00 & 14500.00 & 9.81936446 \\
\hline 9000.00 & 55.00 & 15000.00 & 9.68056646 \\
\hline 9000.00 & 60.00 & 4000.00 & 33.91100412 \\
\hline 8500.00 & 60.00 & 5000.00 & 28.02705954 \\
\hline 9000.00 & 60.00 & 5500.00 & 25.93529936 \\
\hline 9000.00 & 60.00 & 6000.00 & 23.87791790 \\
\hline 7500.00 & 60.00 & 7000.00 & 19.91308295 \\
\hline 8500.00 & 60.00 & 7500.00 & 19.09126261 \\
\hline 8500.00 & 60.00 & 8000.00 & 17.89502693 \\
\hline 8500.00 & 60.00 & 8500.00 & 17.91547748 \\
\hline 6000.00 & 60.00 & 9000.00 & 15.78617020 \\
\hline 7500.00 & 60.00 & 9500.00 & 15.73163665 \\
\hline 7000.00 & 60.00 & 10000.00 & 14.76155159 \\
\hline 6500.00 & 60.00 & 11000.00 & 13.22636220 \\
\hline 9000.00 & 60.00 & 12500.00 & 12.47322139 \\
\hline 8500.00 & 60.00 & 13500.00 & 11.46769850 \\
\hline 8000.00 & 60.00 & 14000.00 & 10.94427444 \\
\hline 8000.00 & 60.00 & 14500.00 & 10.58240835 \\
\hline 7500.00 & 60.00 & 15000.00 & 10.11123714 \\
\hline 8000.00 & 65.00 & 3000.00 & 41.67838704 \\
\hline 9000.00 & 65.00 & 3500.00 & 39.71567798 \\
\hline
\end{tabular}




\begin{tabular}{|c|c|c|c|}
\hline 9000.00 & 65.00 & 4000.00 & 36.28190184 \\
\hline 9000.00 & 65.00 & 6000.00 & 25.60475902 \\
\hline 7500.00 & 65.00 & 7000.00 & 21.31180703 \\
\hline 9000.00 & 65.00 & 7500.00 & 20.69253131 \\
\hline 8500.00 & 65.00 & 8000.00 & 19.23150777 \\
\hline 8500.00 & 65.00 & 8500.00 & 19.25617400 \\
\hline 8500.00 & 65.00 & 9000.00 & 18.19170292 \\
\hline 8500.00 & 65.00 & 9500.00 & 17.24380444 \\
\hline 8000.00 & 65.00 & 10000.00 & 16.22448977 \\
\hline 7500.00 & 65.00 & 10500.00 & 15.26235054 \\
\hline 7000.00 & 65.00 & 11000.00 & 14.38478577 \\
\hline 6500.00 & 65.00 & 11500.00 & 13.54077763 \\
\hline 9000.00 & 65.00 & 12000.00 & 13.89934392 \\
\hline 6000.00 & 65.00 & 13000.00 & 11.76224492 \\
\hline 7500.00 & 65.00 & 13500.00 & 11.96658086 \\
\hline 8000.00 & 70.00 & 4000.00 & 37.48006237 \\
\hline 8000.00 & 70.00 & 4500.00 & 33.87490296 \\
\hline 8000.00 & 70.00 & 6000.00 & 26.53643078 \\
\hline 9000.00 & 70.00 & 6500.00 & 25.27862597 \\
\hline 7500.00 & 70.00 & 7500.00 & 21.21480233 \\
\hline 9000.00 & 70.00 & 9000.00 & 19.61250166 \\
\hline 8500.00 & 70.00 & 9500.00 & 18.40820477 \\
\hline 7500.00 & 70.00 & 10000.00 & 17.09548893 \\
\hline 8000.00 & 70.00 & 10500.00 & 16.48927385 \\
\hline 7500.00 & 70.00 & 11000.00 & 15.53284326 \\
\hline 7500.00 & 70.00 & 11500.00 & 14.87058034 \\
\hline 7000.00 & 70.00 & 12000.00 & 14.03630482 \\
\hline 8500.00 & 70.00 & 12500.00 & 14.08586423 \\
\hline 6500.00 & 70.00 & 13000.00 & 12.73817018 \\
\hline 7500.00 & 70.00 & 14000.00 & 12.28356638 \\
\hline 7000.00 & 70.00 & 15000.00 & 11.31183870 \\
\hline
\end{tabular}




\begin{tabular}{|c|c|c|c|c|}
\hline 8500.00 & 75.00 & 4000.00 & 40.23605261 & 17 \\
\hline 9000.00 & 75.00 & 5000.00 & 34.11242857 & 18 \\
\hline 8000.00 & 75.00 & 5500.00 & 30.35902782 & 16 \\
\hline 7000.00 & 75.00 & 6500.00 & 25.07760899 & 14 \\
\hline 7500.00 & 75.00 & 9000.00 & 20.02470209 & 15 \\
\hline 8500.00 & 75.00 & 9500.00 & 19.57484005 & 17 \\
\hline 9000.00 & 75.00 & 10000.00 & 18.76063163 & 18 \\
\hline 8000.00 & 75.00 & 10500.00 & 17.50974708 & 16 \\
\hline 8000.00 & 75.00 & 11000.00 & 16.69860892 & 16 \\
\hline 8500.00 & 75.00 & 14000.00 & 13.38243844 & 17 \\
\hline 8500.00 & 75.00 & 14500.00 & 12.93912151 & 17 \\
\hline 8000.00 & 80.00 & 3500.00 & 45.32291227 & 16 \\
\hline 9000.00 & 80.00 & 4000.00 & 42.99213444 & 18 \\
\hline 7500.00 & 80.00 & 5000.00 & 34.20349096 & 15 \\
\hline 9000.00 & 80.00 & 8500.00 & 23.24792133 & 18 \\
\hline 8500.00 & 80.00 & 9000.00 & 21.74004471 & 17 \\
\hline 9000.00 & 80.00 & 10500.00 & 18.90786929 & 18 \\
\hline 8000.00 & 80.00 & 11000.00 & 17.66222305 & 16 \\
\hline 8500.00 & 80.00 & 11500.00 & 17.09269487 & 17 \\
\hline 7500.00 & 80.00 & 12000.00 & 15.96325812 & 15 \\
\hline 7500.00 & 80.00 & 13000.00 & 14.73944260 & 15 \\
\hline 8500.00 & 80.00 & 14000.00 & 14.13117555 & 17 \\
\hline 9000.00 & 80.00 & 14500.00 & 13.81288456 & 18 \\
\hline 9000.00 & 80.00 & 15000.00 & 13.37537505 & 18 \\
\hline 8000.00 & 85.00 & 5500.00 & 33.48642541 & 16 \\
\hline 8500.00 & 85.00 & 6000.00 & 31.43887428 & 17 \\
\hline 8500.00 & 85.00 & 7000.00 & 27.31266384 & 17 \\
\hline 9000.00 & 85.00 & 7500.00 & 25.97282399 & 18 \\
\hline 9000.00 & 85.00 & 8000.00 & 24.42008940 & 18 \\
\hline 9000.00 & 85.00 & 9000.00 & 23.14359260 & 18 \\
\hline 8500.00 & 85.00 & 11000.00 & 18.83135688 & 17 \\
\hline
\end{tabular}




\begin{tabular}{|c|c|c|c|}
\hline 8000.00 & 85.00 & 12000.00 & 17.02426577 \\
\hline 8500.00 & 85.00 & 12500.00 & 16.57081364 \\
\hline 8000.00 & 85.00 & 13000.00 & 15.73417796 \\
\hline 9000.00 & 85.00 & 15000.00 & 14.06982100 \\
\hline 9000.00 & 90.00 & 3000.00 & 55.47540617 \\
\hline 8500.00 & 90.00 & 4000.00 & 46.30096851 \\
\hline 8500.00 & 90.00 & 5500.00 & 35.60297263 \\
\hline 8500.00 & 90.00 & 6500.00 & 30.55159620 \\
\hline 9000.00 & 90.00 & 9500.00 & 23.05127058 \\
\hline 7000.00 & 90.00 & 11000.00 & 18.69782759 \\
\hline 9000.00 & 90.00 & 11500.00 & 19.11813028 \\
\hline 7000.00 & 90.00 & 12000.00 & 17.19234477 \\
\hline 8500.00 & 90.00 & 13000.00 & 16.72586017 \\
\hline 7500.00 & 90.00 & 14000.00 & 15.06795621 \\
\hline 9000.00 & 95.00 & 4000.00 & 49.10195804 \\
\hline 8500.00 & 95.00 & 6000.00 & 34.27308674 \\
\hline 7500.00 & 95.00 & 6500.00 & 30.58978422 \\
\hline 7000.00 & 95.00 & 8000.00 & 24.67323060 \\
\hline 7000.00 & 95.00 & 11500.00 & 18.65644681 \\
\hline 9000.00 & 95.00 & 13000.00 & 17.72097845 \\
\hline 7500.00 & 95.00 & 13500.00 & 16.33713065 \\
\hline 8000.00 & 100.00 & 4000.00 & 48.96325282 \\
\hline 9000.00 & 100.00 & 7000.00 & 31.44859499 \\
\hline 9000.00 & 100.00 & 9000.00 & 26.43636962 \\
\hline 9000.00 & 100.00 & 10000.00 & 23.89589485 \\
\hline 9000.00 & 100.00 & 13500.00 & 17.80632384 \\
\hline 8500.00 & 100.00 & 14000.00 & 16.93760518 \\
\hline 8000.00 & 100.00 & 14500.00 & 16.11611448 \\
\hline 8500.00 & 100.00 & 15000.00 & 15.83359095 \\
\hline
\end{tabular}

The following are the tabulated results for the points of diminishing returns, where one exists for a combination of initial mass, power, and specific impulse, sorted by 
specific impulse.

\begin{tabular}{|c|c|c|c|c|}
\hline $\mathrm{mo} \quad(\mathrm{kg})$ & PO (kW) & ISP ( $\mathrm{sec})$ & Value (kg/day) & Po Index \\
\hline 6000.00 & 35.00 & 3000.00 & 23.47837833 & 12 \\
\hline 8000.00 & 40.00 & 3000.00 & 27.83455177 & 16 \\
\hline 9000.00 & 50.00 & 3000.00 & 34.46897729 & 18 \\
\hline 7500.00 & 55.00 & 3000.00 & 35.90675143 & 15 \\
\hline 8000.00 & 65.00 & 3000.00 & 41.67838704 & 16 \\
\hline 9000.00 & 90.00 & 3000.00 & 55.47540617 & 18 \\
\hline 8500.00 & 30.00 & 3500.00 & 20.01228621 & 17 \\
\hline 6500.00 & 35.00 & 3500.00 & 22.02911109 & 13 \\
\hline 7500.00 & 40.00 & 3500.00 & 25.37035181 & 15 \\
\hline 8500.00 & 45.00 & 3500.00 & 28.75340387 & 17 \\
\hline 8000.00 & 50.00 & 3500.00 & 31.09072491 & 16 \\
\hline 9000.00 & 65.00 & 3500.00 & 39.71567798 & 18 \\
\hline 8000.00 & 80.00 & 3500.00 & 45.32291227 & 16 \\
\hline 8500.00 & 30.00 & 4000.00 & 18.13885230 & 17 \\
\hline 8500.00 & 40.00 & 4000.00 & 23.65065887 & 17 \\
\hline 7500.00 & 50.00 & 4000.00 & 28.00845546 & 15 \\
\hline 6000.00 & 55.00 & 4000.00 & 28.62257720 & 12 \\
\hline 9000.00 & 60.00 & 4000.00 & 33.91100412 & 18 \\
\hline 9000.00 & 65.00 & 4000.00 & 36.28190184 & 18 \\
\hline 8000.00 & 70.00 & 4000.00 & 37.48006237 & 16 \\
\hline 8500.00 & 75.00 & 4000.00 & 40.23605261 & 17 \\
\hline 9000.00 & 80.00 & 4000.00 & 42.99213444 & 18 \\
\hline 8500.00 & 90.00 & 4000.00 & 46.30096851 & 17 \\
\hline 9000.00 & 95.00 & 4000.00 & 49.10195804 & 18 \\
\hline 8000.00 & 100.00 & 4000.00 & 48.96325282 & 16 \\
\hline 7000.00 & 30.00 & 4500.00 & 15.99589599 & 14 \\
\hline 8500.00 & 35.00 & 4500.00 & 18.83008803 & 17 \\
\hline 8500.00 & 45.00 & 4500.00 & 23.65940873 & 17 \\
\hline
\end{tabular}




\begin{tabular}{|c|c|c|c|}
\hline 8500.00 & 55.00 & 4500.00 & 28.16547567 \\
\hline 8000.00 & 70.00 & 4500.00 & 33.87490296 \\
\hline 7000.00 & 30.00 & 5000.00 & 14.68268270 \\
\hline 7500.00 & 35.00 & 5000.00 & 17.10355142 \\
\hline 9000.00 & 40.00 & 5000.00 & 19.73029656 \\
\hline 7500.00 & 45.00 & 5000.00 & 21.40682874 \\
\hline 8500.00 & 50.00 & 5000.00 & 23.95450876 \\
\hline 8500.00 & 60.00 & 5000.00 & 28.02705954 \\
\hline 9000.00 & 75.00 & 5000.00 & 34.11242857 \\
\hline 7500.00 & 80.00 & 5000.00 & 34.20349096 \\
\hline 6500.00 & 30.00 & 5500.00 & 13.26239802 \\
\hline 7000.00 & 35.00 & 5500.00 & 15.43272916 \\
\hline 8500.00 & 40.00 & 5500.00 & 17.85046658 \\
\hline 7000.00 & 45.00 & 5500.00 & 19.31383037 \\
\hline 8500.00 & 55.00 & 5500.00 & 23.81613038 \\
\hline 9000.00 & 60.00 & 5500.00 & 25.93529936 \\
\hline 8000.00 & 75.00 & 5500.00 & 30.35902782 \\
\hline 8000.00 & 85.00 & 5500.00 & 33.48642541 \\
\hline 8500.00 & 90.00 & 5500.00 & 35.60297263 \\
\hline 7000.00 & 30.00 & 6000.00 & 12.32509269 \\
\hline 6500.00 & 35.00 & 6000.00 & 14.03768783 \\
\hline 7000.00 & 40.00 & 6000.00 & 16.04366841 \\
\hline 9000.00 & 45.00 & 6000.00 & 18.40834610 \\
\hline 9000.00 & 50.00 & 6000.00 & 20.29533875 \\
\hline 9000.00 & 60.00 & 6000.00 & 23.87791790 \\
\hline 9000.00 & 65.00 & 6000.00 & 25.60475902 \\
\hline 8000.00 & 70.00 & 6000.00 & 26.53643078 \\
\hline 8500.00 & 85.00 & 6000.00 & 31.43887428 \\
\hline 8500.00 & 95.00 & 6000.00 & 34.27308674 \\
\hline 5000.00 & 30.00 & 6500.00 & 10.85170700 \\
\hline 6000.00 & 35.00 & 6500.00 & 12.82931998 \\
\hline
\end{tabular}




\begin{tabular}{|c|c|c|c|}
\hline 7000.00 & 40.00 & 6500.00 & 14.80290800 \\
\hline 8000.00 & 45.00 & 6500.00 & 16.77247097 \\
\hline 6500.00 & 50.00 & 6500.00 & 17.78342729 \\
\hline 9000.00 & 70.00 & 6500.00 & 25.27862597 \\
\hline 7000.00 & 75.00 & 6500.00 & 25.07760899 \\
\hline 8500.00 & 90.00 & 6500.00 & 30.55159620 \\
\hline 7500.00 & 95.00 & 6500.00 & 30.58978422 \\
\hline 7000.00 & 30.00 & 7000.00 & 10.64237955 \\
\hline 8500.00 & 35.00 & 7000.00 & 12.55024657 \\
\hline 6500.00 & 40.00 & 7000.00 & 13.61910706 \\
\hline 6500.00 & 45.00 & 7000.00 & 15.14981005 \\
\hline 7500.00 & 50.00 & 7000.00 & 17.01493166 \\
\hline 6500.00 & 55.00 & 7000.00 & 17.95020034 \\
\hline 7500.00 & 60.00 & 7000.00 & 19.91308295 \\
\hline 7500.00 & 65.00 & 7000.00 & 21.31180703 \\
\hline 8500.00 & 85.00 & 7000.00 & 27.31266384 \\
\hline 9000.00 & 100.00 & 7000.00 & 31.44859499 \\
\hline 8500.00 & 30.00 & 7500.00 & 10.20290688 \\
\hline 8000.00 & 35.00 & 7500.00 & 11.66265043 \\
\hline 6000.00 & 40.00 & 7500.00 & 12.56481152 \\
\hline 6500.00 & 45.00 & 7500.00 & 14.14664054 \\
\hline 7500.00 & 50.00 & 7500.00 & 15.89134590 \\
\hline 7500.00 & 55.00 & 7500.00 & 17.31388861 \\
\hline 8500.00 & 60.00 & 7500.00 & 19.09126261 \\
\hline 9000.00 & 65.00 & 7500.00 & 20.69253131 \\
\hline 7500.00 & 70.00 & 7500.00 & 21.21480233 \\
\hline 9000.00 & 85.00 & 7500.00 & 25.97282399 \\
\hline 8500.00 & 30.00 & 8000.00 & 9.59088304 \\
\hline 7500.00 & 35.00 & 8000.00 & 10.86850773 \\
\hline 5500.00 & 40.00 & 8000.00 & 11.60942127 \\
\hline 6500.00 & 45.00 & 8000.00 & 13.26118185 \\
\hline
\end{tabular}




\begin{tabular}{|c|c|c|c|}
\hline 7000.00 & 50.00 & 8000.00 & 14.74057822 \\
\hline 8000.00 & 55.00 & 8000.00 & 16.39852059 \\
\hline 8500.00 & 60.00 & 8000.00 & 17.89502693 \\
\hline 8500.00 & 65.00 & 8000.00 & 19.23150777 \\
\hline 9000.00 & 85.00 & 8000.00 & 24.42008940 \\
\hline 7000.00 & 95.00 & 8000.00 & 24.67323060 \\
\hline 8000.00 & 30.00 & 8500.00 & 9.54186965 \\
\hline 7500.00 & 35.00 & 8500.00 & 10.88680685 \\
\hline 5500.00 & 40.00 & 8500.00 & 11.62875244 \\
\hline 6500.00 & 45.00 & 8500.00 & 13.27723013 \\
\hline 7000.00 & 50.00 & 8500.00 & 14.77025818 \\
\hline 8000.00 & 55.00 & 8500.00 & 16.41742772 \\
\hline 8500.00 & 60.00 & 8500.00 & 17.91547748 \\
\hline 8500.00 & 65.00 & 8500.00 & 19.25617400 \\
\hline 9000.00 & 80.00 & 8500.00 & 23.24792133 \\
\hline 9000.00 & 30.00 & 9000.00 & 9.15951217 \\
\hline 5000.00 & 40.00 & 9000.00 & 10.79179387 \\
\hline 6000.00 & 45.00 & 9000.00 & 12.39207382 \\
\hline 6500.00 & 50.00 & 9000.00 & 13.77787066 \\
\hline 6000.00 & 60.00 & 9000.00 & 15.78617020 \\
\hline 8500.00 & 65.00 & 9000.00 & 18.19170292 \\
\hline 9000.00 & 70.00 & 9000.00 & 19.61250166 \\
\hline 7500.00 & 75.00 & 9000.00 & 20.02470209 \\
\hline 8500.00 & 80.00 & 9000.00 & 21.74004471 \\
\hline 9000.00 & 85.00 & 9000.00 & 23.14359260 \\
\hline 9000.00 & 100.00 & 9000.00 & 26.43636962 \\
\hline 7000.00 & 30.00 & 9500.00 & 8.43479508 \\
\hline 8000.00 & 35.00 & 9500.00 & 9.87724143 \\
\hline 5500.00 & 45.00 & 9500.00 & 11.55410671 \\
\hline 8000.00 & 50.00 & 9500.00 & 13.57301300 \\
\hline 8500.00 & 55.00 & 9500.00 & 14.90969311 \\
\hline
\end{tabular}




\begin{tabular}{|c|c|c|c|}
\hline 7500.00 & 60.00 & 9500.00 & 15.73163665 \\
\hline 8500.00 & 65.00 & 9500.00 & 17.24380444 \\
\hline 8500.00 & 70.00 & 9500.00 & 18.40820477 \\
\hline 8500.00 & 75.00 & 9500.00 & 19.57484005 \\
\hline 9000.00 & 90.00 & 9500.00 & 23.05127058 \\
\hline 6500.00 & 30.00 & 10000.00 & 7.95041073 \\
\hline 6500.00 & 35.00 & 10000.00 & 9.12280962 \\
\hline 7500.00 & 40.00 & 10000.00 & 10.50373963 \\
\hline 8500.00 & 45.00 & 10000.00 & 11.87863155 \\
\hline 6000.00 & 50.00 & 10000.00 & 12.22864054 \\
\hline 9000.00 & 55.00 & 10000.00 & 14.30870123 \\
\hline 7000.00 & 60.00 & 10000.00 & 14.76155159 \\
\hline 8000.00 & 65.00 & 10000.00 & 16.22448977 \\
\hline 7500.00 & 70.00 & 10000.00 & 17.09548893 \\
\hline 9000.00 & 75.00 & 10000.00 & 18.76063163 \\
\hline 9000.00 & 100.00 & 10000.00 & 23.89589485 \\
\hline 8500.00 & 30.00 & 10500.00 & 7.84142696 \\
\hline 7500.00 & 35.00 & 10500.00 & 8.89061839 \\
\hline 7000.00 & 40.00 & 10500.00 & 9.91243620 \\
\hline 5000.00 & 45.00 & 10500.00 & 10.23910901 \\
\hline 7500.00 & 50.00 & 10500.00 & 12.18176231 \\
\hline 7500.00 & 65.00 & 10500.00 & 15.26235054 \\
\hline 8000.00 & 70.00 & 10500.00 & 16.48927385 \\
\hline 8000.00 & 75.00 & 10500.00 & 17.50974708 \\
\hline 9000.00 & 80.00 & 10500.00 & 18.90786929 \\
\hline 7000.00 & 30.00 & 11000.00 & 7.32032424 \\
\hline 7000.00 & 35.00 & 11000.00 & 8.41531331 \\
\hline 6500.00 & 40.00 & 11000.00 & 9.35797587 \\
\hline 6500.00 & 50.00 & 11000.00 & 11.34195358 \\
\hline 6000.00 & 55.00 & 11000.00 & 12.07011340 \\
\hline 6500.00 & 60.00 & 11000.00 & 13.22636220 \\
\hline
\end{tabular}




\begin{tabular}{|c|c|c|c|}
\hline 7000.00 & 65.00 & 11000.00 & 14.38478577 \\
\hline 7500.00 & 70.00 & 11000.00 & 15.53284326 \\
\hline 8000.00 & 75.00 & 11000.00 & 16.69860892 \\
\hline 8000.00 & 80.00 & 11000.00 & 17.66222305 \\
\hline 8500.00 & 85.00 & 11000.00 & 18.83135688 \\
\hline 7000.00 & 90.00 & 11000.00 & 18.69782759 \\
\hline 7000.00 & 30.00 & 11500.00 & 7.02123136 \\
\hline 8000.00 & 35.00 & 11500.00 & 8.21466075 \\
\hline 8500.00 & 40.00 & 11500.00 & 9.34404681 \\
\hline 7500.00 & 45.00 & 11500.00 & 10.18092125 \\
\hline 6500.00 & 50.00 & 11500.00 & 10.87009618 \\
\hline 5500.00 & 55.00 & 11500.00 & 11.32468077 \\
\hline 6500.00 & 65.00 & 11500.00 & 13.54077763 \\
\hline 7500.00 & 70.00 & 11500.00 & 14.87058034 \\
\hline 8500.00 & 80.00 & 11500.00 & 17.09269487 \\
\hline 9000.00 & 90.00 & 11500.00 & 19.11813028 \\
\hline 7000.00 & 95.00 & 11500.00 & 18.65644681 \\
\hline 6500.00 & 30.00 & 12000.00 & 6.66579675 \\
\hline 7000.00 & 35.00 & 12000.00 & 7.73863014 \\
\hline 9000.00 & 40.00 & 12000.00 & 9.04163814 \\
\hline 8000.00 & 45.00 & 12000.00 & 9.88731030 \\
\hline 9000.00 & 50.00 & 12000.00 & 11.04486661 \\
\hline 7500.00 & 55.00 & 12000.00 & 11.62998652 \\
\hline 9000.00 & 65.00 & 12000.00 & 13.89934392 \\
\hline 7000.00 & 70.00 & 12000.00 & 14.03630482 \\
\hline 7500.00 & 80.00 & 12000.00 & 15.96325812 \\
\hline 8000.00 & 85.00 & 12000.00 & 17.02426577 \\
\hline 7000.00 & 90.00 & 12000.00 & 17.19234477 \\
\hline 9000.00 & 30.00 & 12500.00 & 6.65913687 \\
\hline 7500.00 & 35.00 & 12500.00 & 7.51557890 \\
\hline 7500.00 & 40.00 & 12500.00 & 8.46639503 \\
\hline
\end{tabular}




\begin{tabular}{|c|c|c|c|}
\hline 7500.00 & 45.00 & 12500.00 & 9.40296178 \\
\hline 7500.00 & 50.00 & 12500.00 & 10.30919043 \\
\hline 9000.00 & 60.00 & 12500.00 & 12.47322139 \\
\hline 8500.00 & 70.00 & 12500.00 & 14.08586423 \\
\hline 8500.00 & 85.00 & 12500.00 & 16.57081364 \\
\hline 8000.00 & 30.00 & 13000.00 & 6.33956313 \\
\hline 7000.00 & 35.00 & 13000.00 & 7.16214210 \\
\hline 8000.00 & 40.00 & 13000.00 & 8.23023921 \\
\hline 7000.00 & 50.00 & 13000.00 & 9.80424891 \\
\hline 9000.00 & 55.00 & 13000.00 & 11.12584226 \\
\hline 6000.00 & 65.00 & 13000.00 & 11.76224492 \\
\hline 6500.00 & 70.00 & 13000.00 & 12.73817018 \\
\hline 7500.00 & 80.00 & 13000.00 & 14.73944260 \\
\hline 8000.00 & 85.00 & 13000.00 & 15.73417796 \\
\hline 8500.00 & 90.00 & 13000.00 & 16.72586017 \\
\hline 9000.00 & 95.00 & 13000.00 & 17.72097845 \\
\hline 6000.00 & 30.00 & 13500.00 & 5.88443297 \\
\hline 7000.00 & 35.00 & 13500.00 & 6.91275558 \\
\hline 8000.00 & 40.00 & 13500.00 & 7.94359873 \\
\hline 9000.00 & 45.00 & 13500.00 & 8.97650401 \\
\hline 9000.00 & 50.00 & 13500.00 & 9.85899109 \\
\hline 9000.00 & 55.00 & 13500.00 & 10.72759342 \\
\hline 8500.00 & 60.00 & 13500.00 & 11.46769850 \\
\hline 7500.00 & 65.00 & 13500.00 & 11.96658086 \\
\hline 7500.00 & 95.00 & 13500.00 & 16.33713065 \\
\hline 9000.00 & 100.00 & 13500.00 & 17.80632384 \\
\hline 5500.00 & 30.00 & 14000.00 & 5.59540044 \\
\hline 6500.00 & 35.00 & 14000.00 & 6.59024356 \\
\hline 6000.00 & 40.00 & 14000.00 & 7.31343571 \\
\hline 7500.00 & 45.00 & 14000.00 & 8.42506566 \\
\hline 7500.00 & 50.00 & 14000.00 & 9.24632415 \\
\hline
\end{tabular}




\begin{tabular}{|c|c|c|c|}
\hline 8000.00 & 60.00 & 14000.00 & 10.94427444 \\
\hline 7500.00 & 70.00 & 14000.00 & 12.28356638 \\
\hline 8500.00 & 75.00 & 14000.00 & 13.38243844 \\
\hline 8500.00 & 80.00 & 14000.00 & 14.13117555 \\
\hline 7500.00 & 90.00 & 14000.00 & 15.06795621 \\
\hline 8500.00 & 100.00 & 14000.00 & 16.93760518 \\
\hline 7000.00 & 30.00 & 14500.00 & 5.61331021 \\
\hline 8500.00 & 35.00 & 14500.00 & 6.61142699 \\
\hline 7500.00 & 40.00 & 14500.00 & 7.33852750 \\
\hline 7500.00 & 45.00 & 14500.00 & 8.14228997 \\
\hline 9000.00 & 50.00 & 14500.00 & 9.19925871 \\
\hline 8000.00 & 55.00 & 14500.00 & 9.81936446 \\
\hline 8000.00 & 60.00 & 14500.00 & 10.58240835 \\
\hline 8500.00 & 75.00 & 14500.00 & 12.93912151 \\
\hline 9000.00 & 80.00 & 14500.00 & 13.81288456 \\
\hline 8000.00 & 100.00 & 14500.00 & 16.11611448 \\
\hline 7000.00 & 30.00 & 15000.00 & 5.42834474 \\
\hline 8000.00 & 35.00 & 15000.00 & 6.35211101 \\
\hline 7000.00 & 40.00 & 15000.00 & 7.02977396 \\
\hline 7000.00 & 50.00 & 15000.00 & 8.53505507 \\
\hline 9000.00 & 55.00 & 15000.00 & 9.68056646 \\
\hline 7500.00 & 60.00 & 15000.00 & 10.11123714 \\
\hline 7000.00 & 70.00 & 15000.00 & 11.31183870 \\
\hline 9000.00 & 80.00 & 15000.00 & 13.37537505 \\
\hline 9000.00 & 85.00 & 15000.00 & 14.06982100 \\
\hline 8500.00 & 100.00 & 15000.00 & 15.83359095 \\
\hline
\end{tabular}

The following is the list of transfer times for the maximum optimum payload delivered per day.

mo (kg) PO (kW) ISP ( $\mathrm{sec}) \quad$ TOF (days) 


$\begin{array}{rrrr}9000.00 & 90.00 & 3000.00 & 113.3603 \\ 8000.00 & 80.00 & 3500.00 & 124.9487 \\ 9000.00 & 95.00 & 4000.00 & 131.4732 \\ 8000.00 & 70.00 & 4500.00 & 176.1163 \\ 7500.00 & 80.00 & 5000.00 & 158.8637 \\ 8500.00 & 90.00 & 5500.00 & 176.1550 \\ 8500.00 & 95.00 & 6000.00 & 182.4436 \\ 7500.00 & 95.00 & 6500.00 & 175.2215 \\ 9000.00 & 100.00 & 7000.00 & 214.0776 \\ 9000.00 & 85.00 & 7500.00 & 268.1470 \\ 7000.00 & 95.00 & 8000.00 & 201.4830 \\ 9000.00 & 80.00 & 8500.00 & 304.8503 \\ 9000.00 & 100.00 & 9000.00 & 258.9634 \\ 9000.00 & 90.00 & 9500.00 & 303.6106 \\ 9000.00 & 100.00 & 10000.00 & 288.1731 \\ 9000.00 & 80.00 & 10500.00 & 379.1793 \\ 8500.00 & 85.00 & 11000.00 & 352.5733 \\ 9000.00 & 90.00 & 11500.00 & 369.6386 \\ 7000.00 & 90.00 & 12000.00 & 299.9125 \\ 8500.00 & 85.00 & 12500.00 & 403.0023 \\ 9000.00 & 95.00 & 13000.00 & 397.2001 \\ 9000.00 & 100.00 & 13500.00 & 392.2133 \\ 8500.00 & 100.00 & 14000.00 & 384.3762 \\ 8000.00 & 100.00 & 14500.00 & 374.4996 \\ 8500.00 & 100.00 & 15000.00 & 412.2946\end{array}$




\section{Appendix D}

\section{Appendix: Far Term Plots}

Figures D.1 through D.3 present the initial mass versus payload mass in low lunar orbit for eleven power levels $100 \mathrm{~kW}$ to $200 \mathrm{~kW}$, by increments of $10 \mathrm{~kW}$.
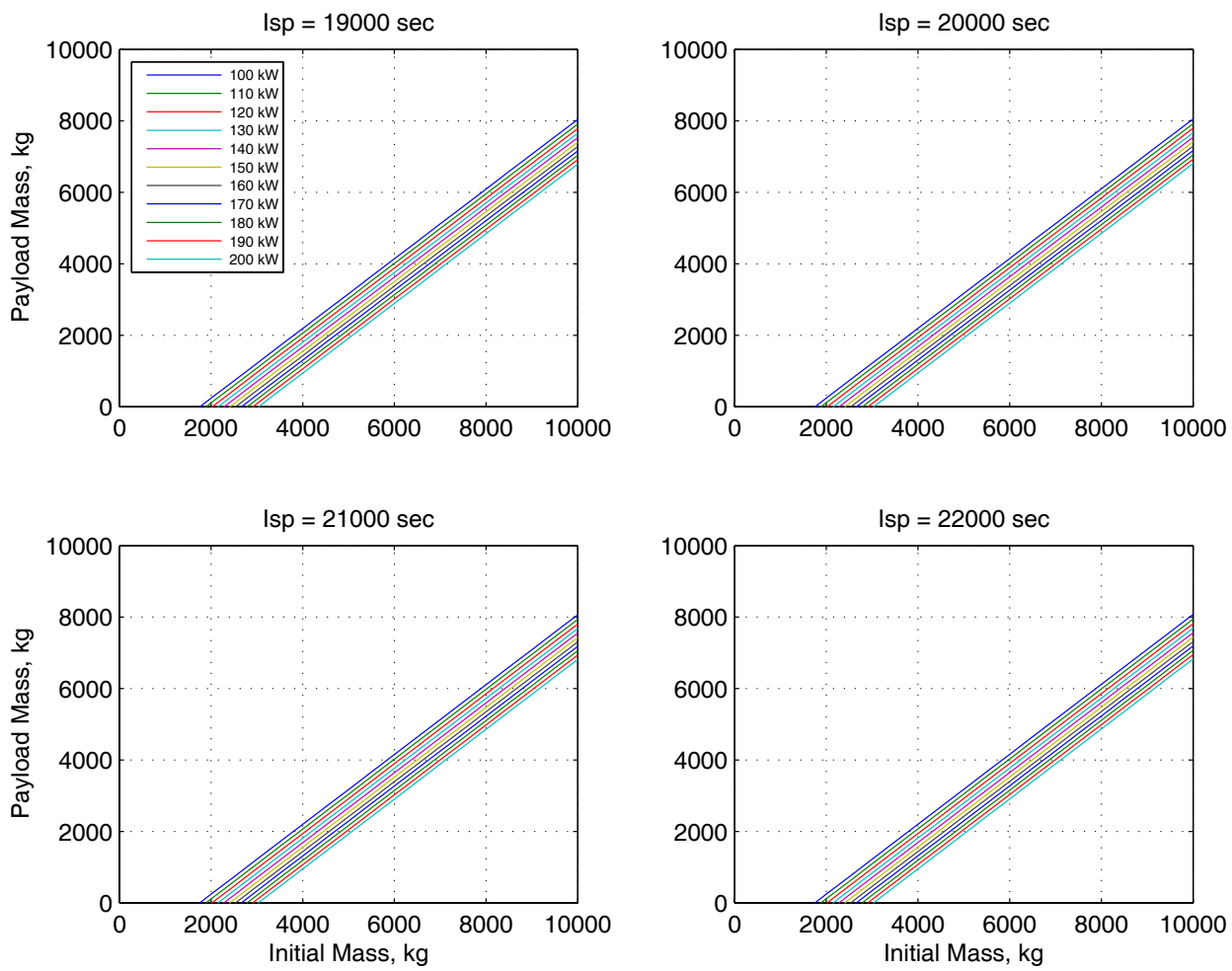

Figure D.1: Initial Mass versus Payload Mass 

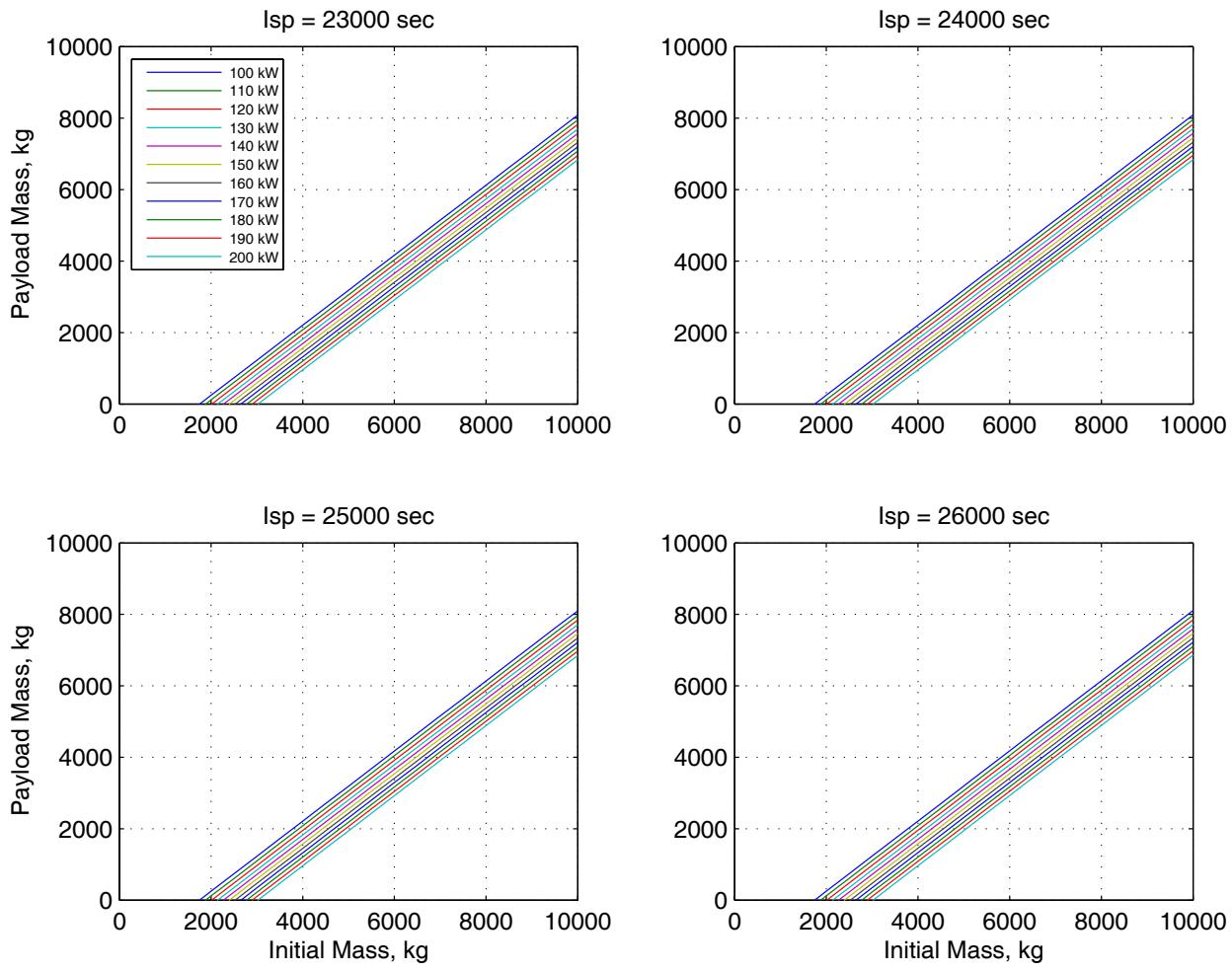

Figure D.2: Initial Mass versus Payload Mass 

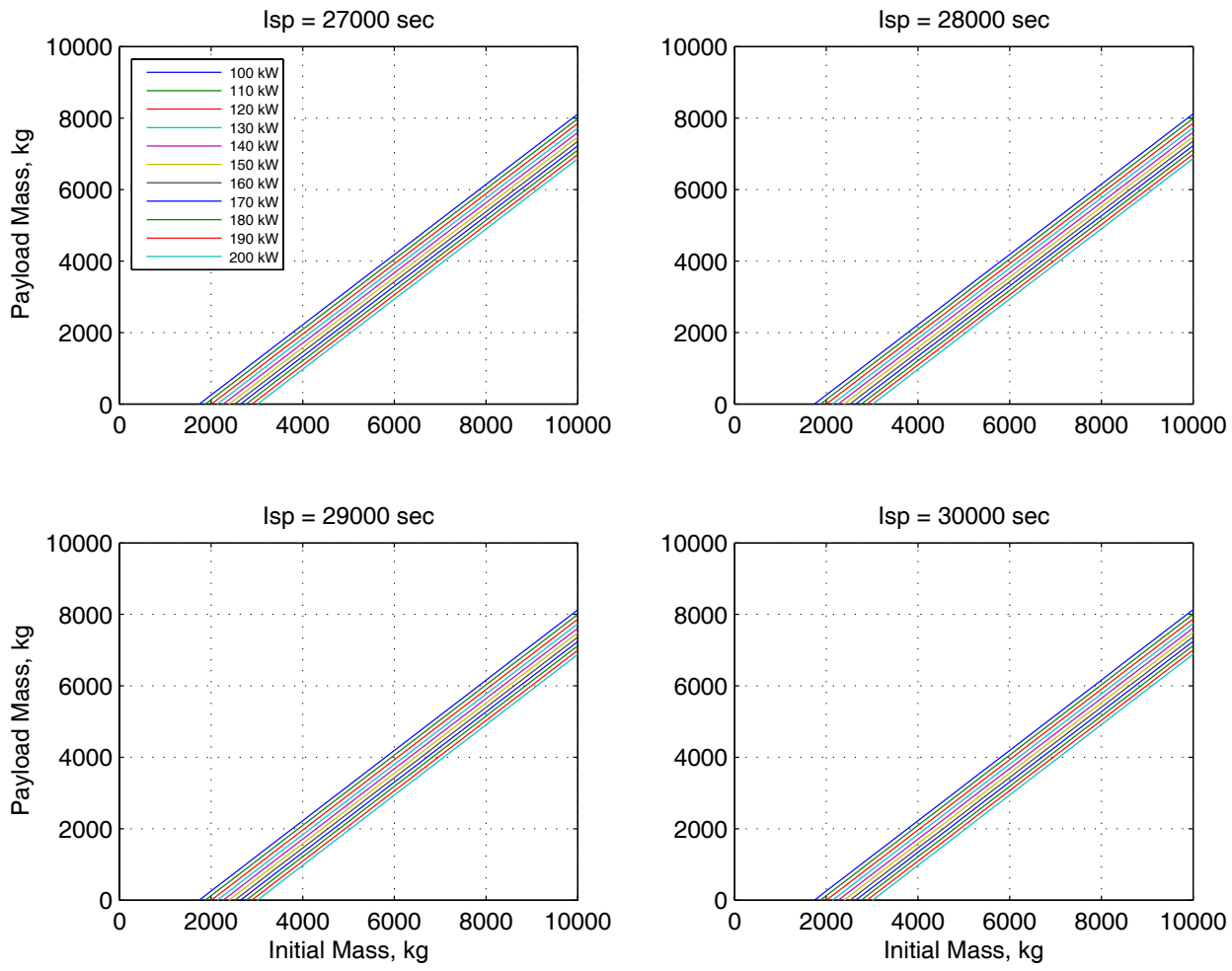

Figure D.3: Initial Mass versus Payload Mass 
Figures D.4 through D.6 present the payload mass per day as a function of initial mass.
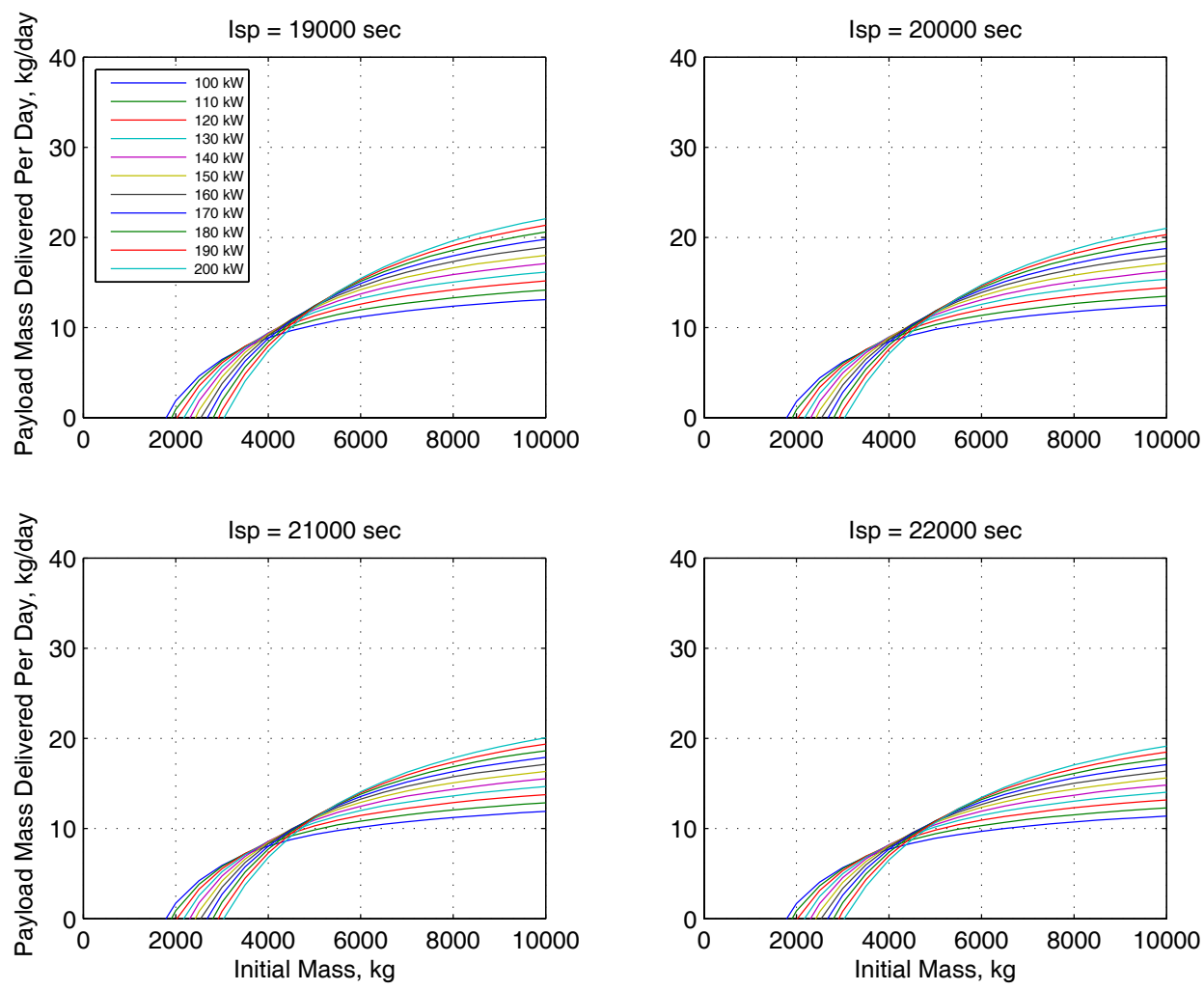

Figure D.4: Initial Mass vs. Payload Mass per Day 

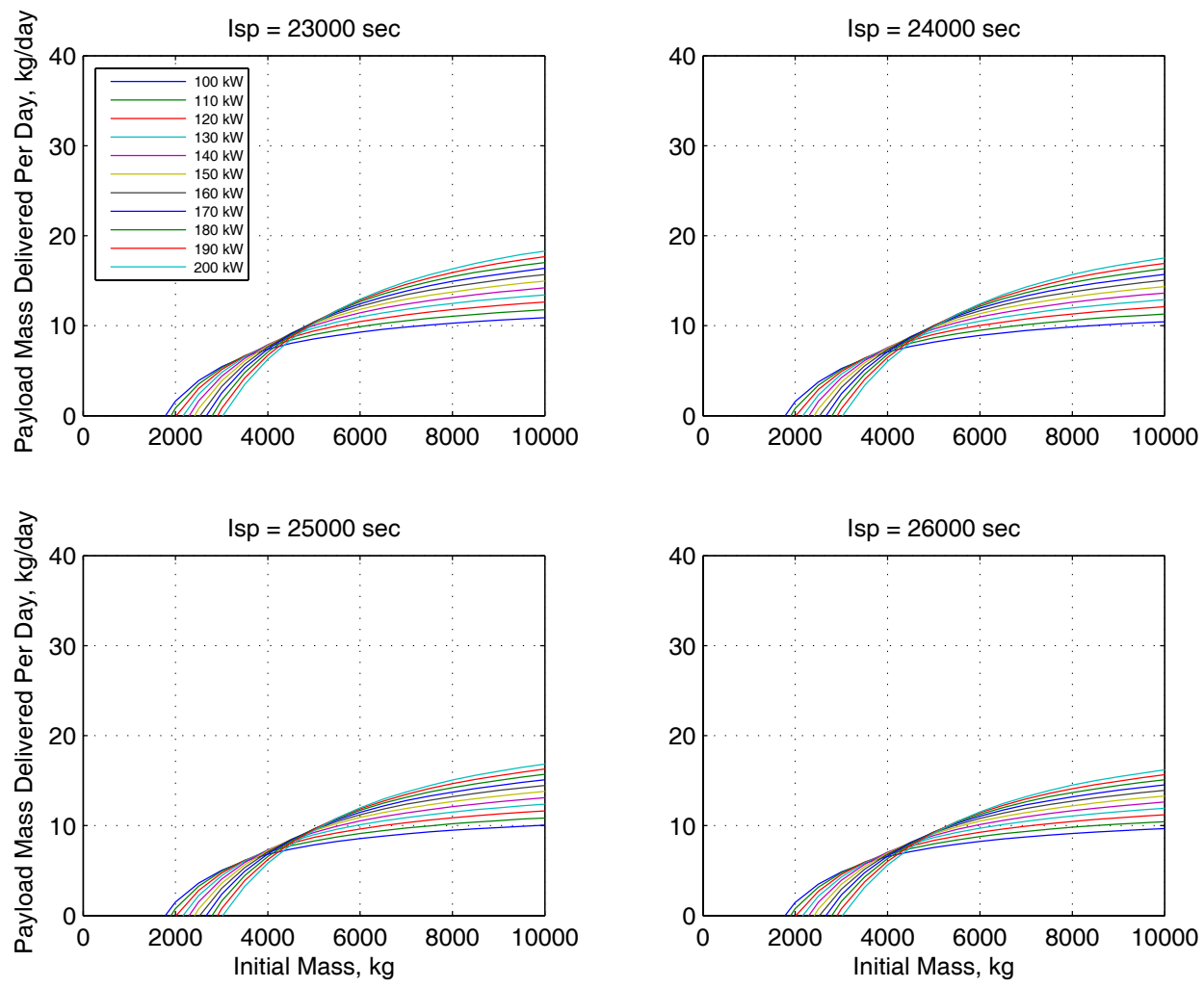

Figure D.5: Initial Mass vs. Payload Mass per Day 

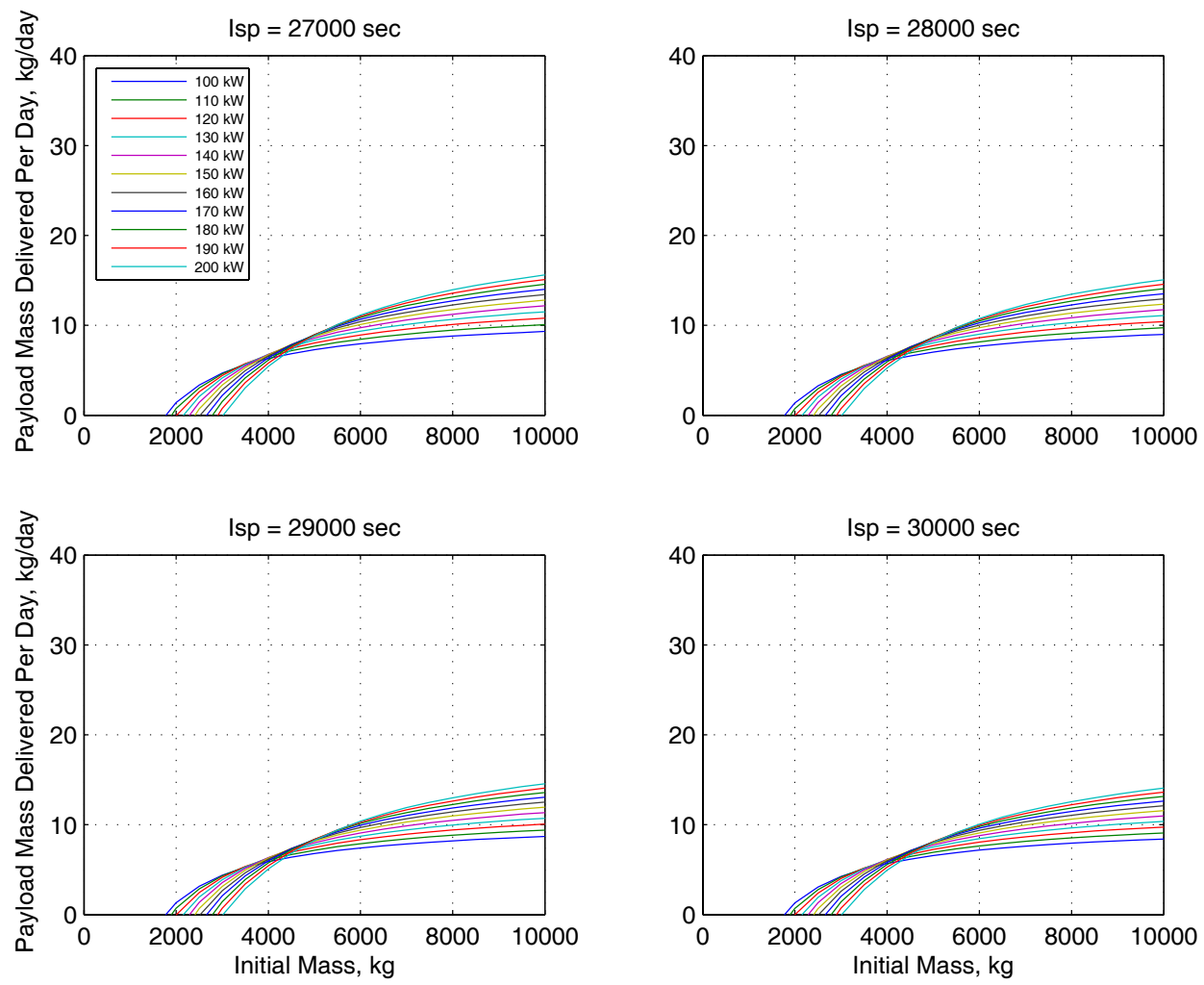

Figure D.6: Initial Mass vs. Payload Mass per Day 

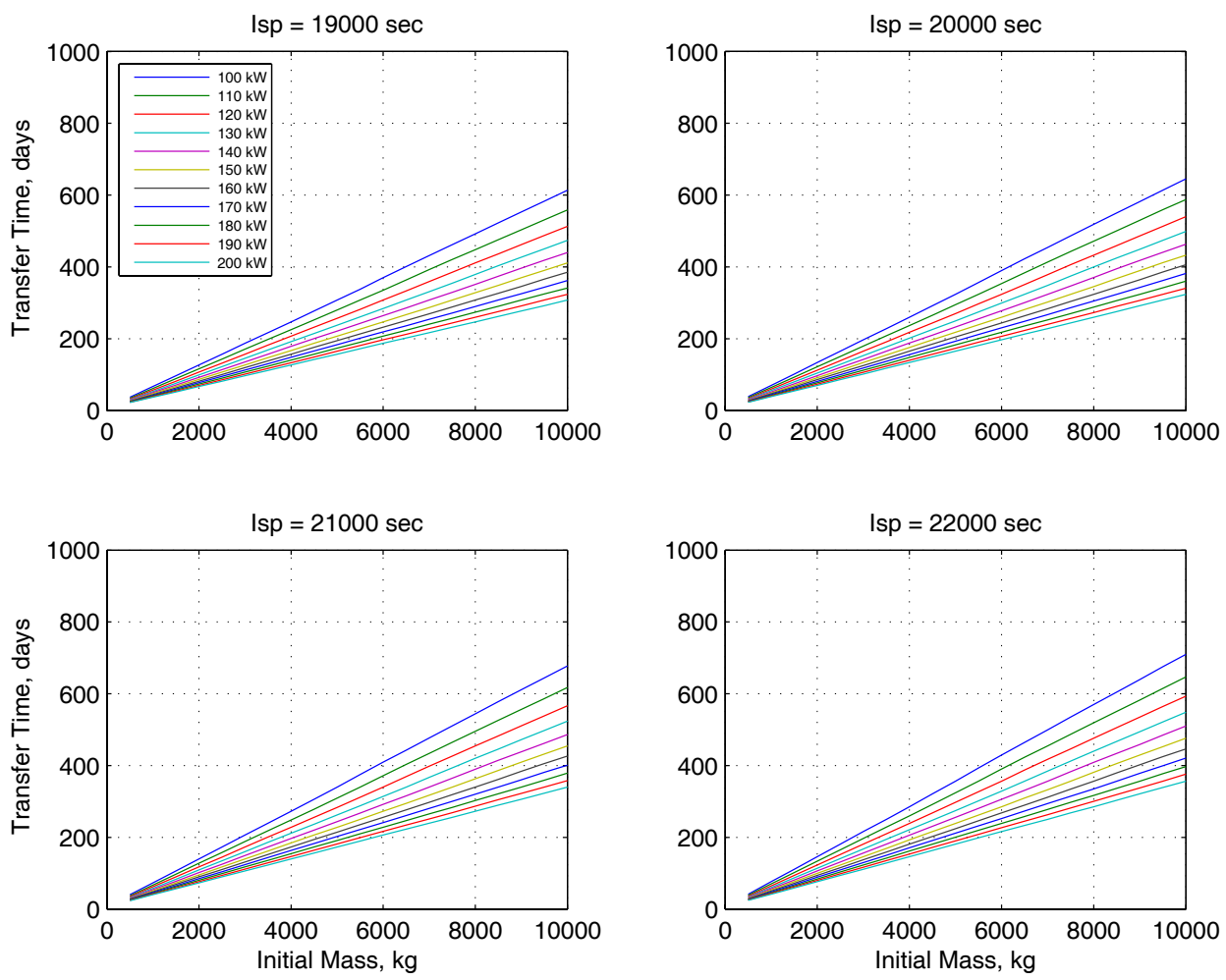

Figure D.7: Initial Mass vs. Payload Mass per Day

Figures D.7 to D.9 highlight the transfer times for a range of $I_{s p}$, from $1250 \mathrm{sec}$ to 3000 sec. 

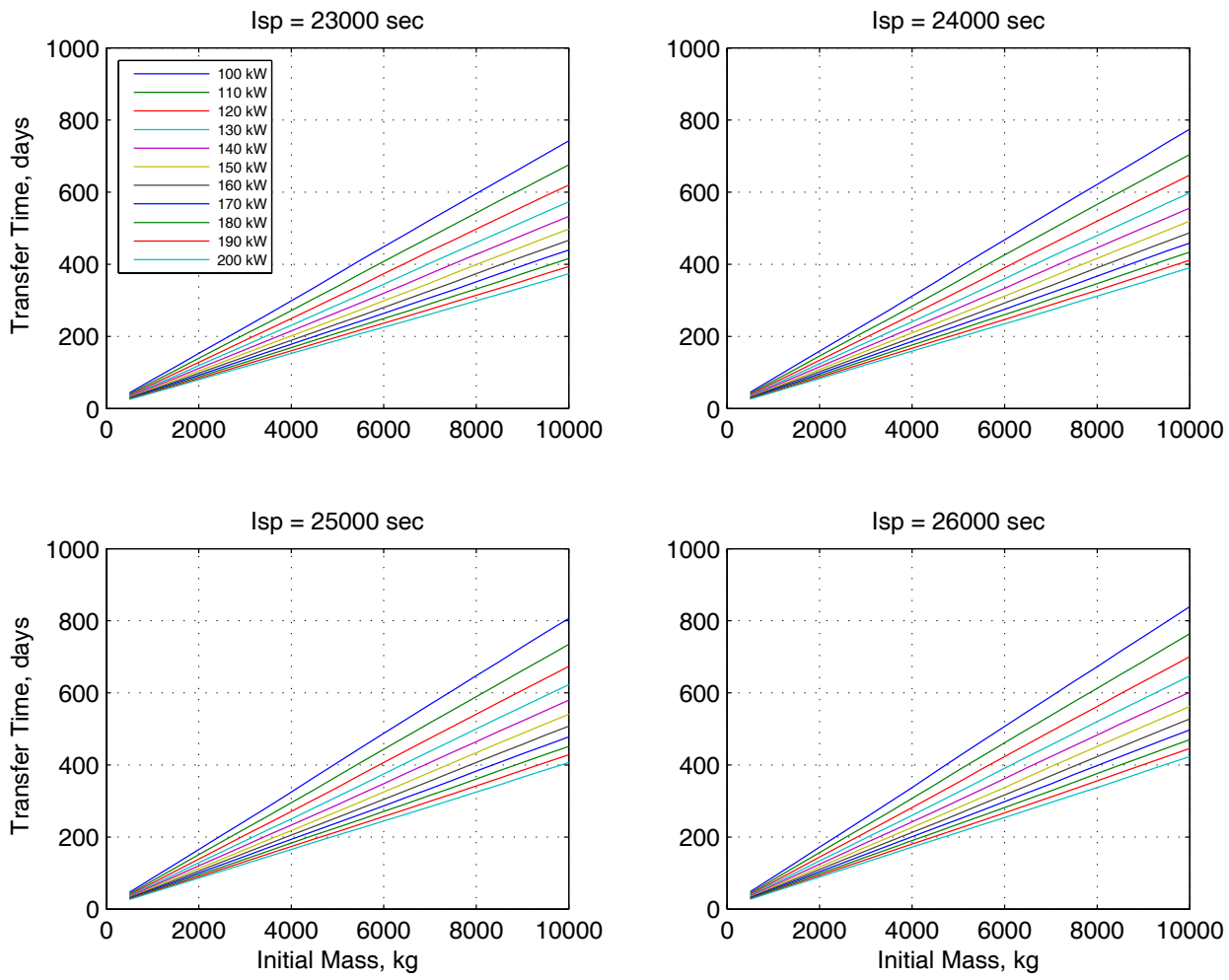

Figure D.8: Initial Mass vs. Payload Mass per Day 

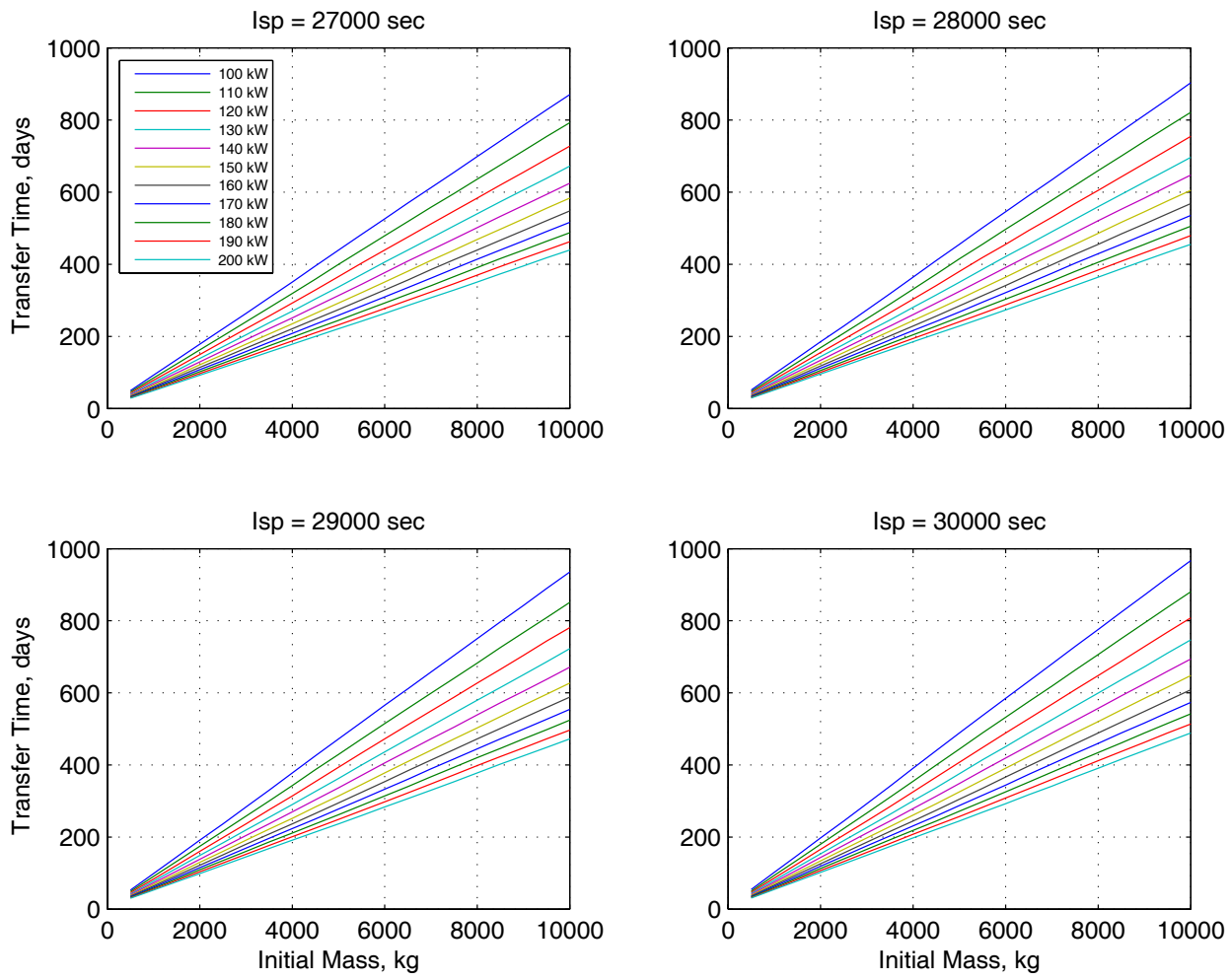

Figure D.9: Initial Mass vs. Payload Mass per Day 
Figures D.10 to D.12 highlight the transfer times for a range of power levels.
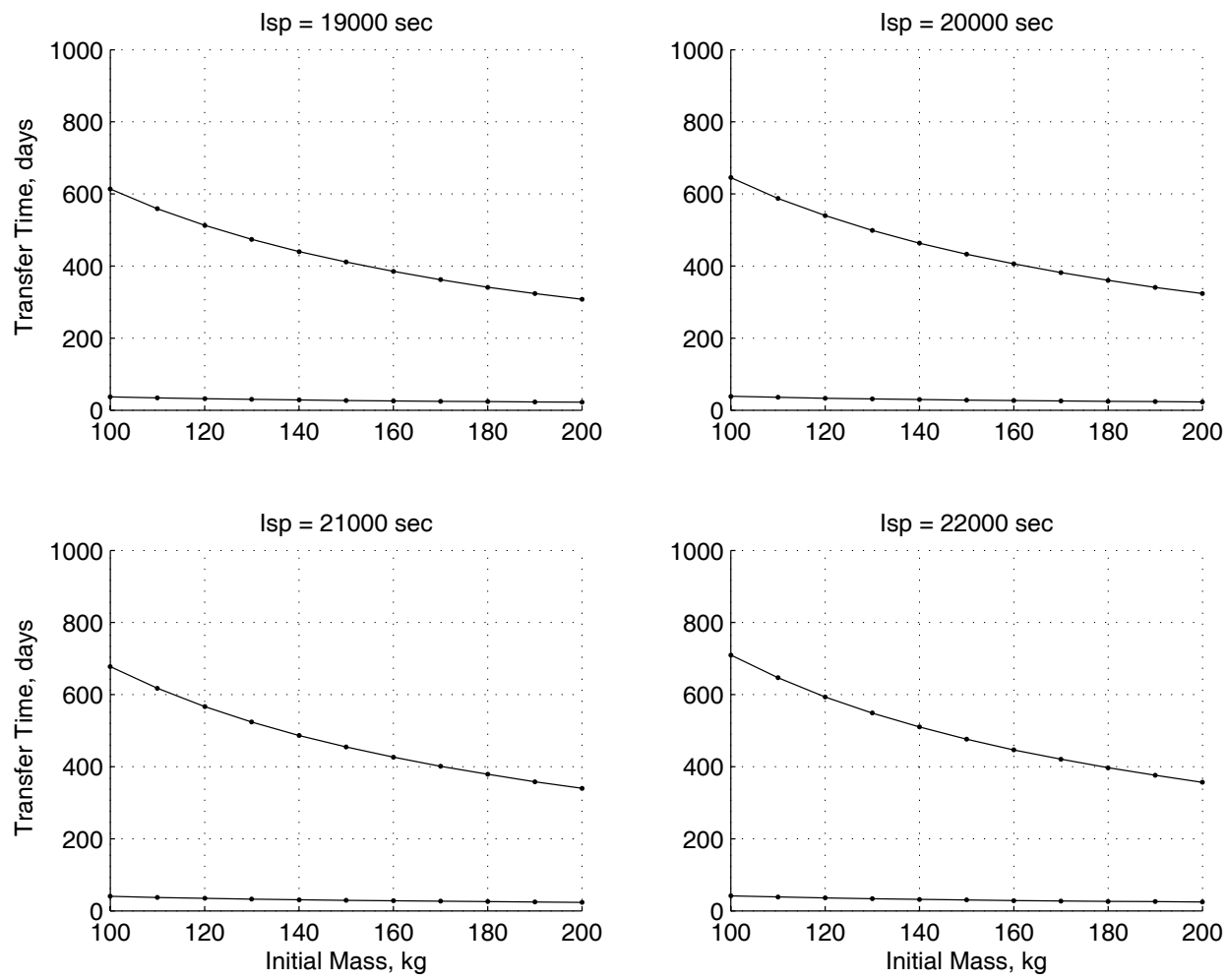

Figure D.10: Power vs. Transfer Time

The following are the tabulated results for the points of diminishing returns, where one exists for a combination of initial mass, power, and specific impulse, sorted by power. There are 34 cases that have a point of diminishing returns for the initial mass versus payload delivered per day curves.

m0 (kg) PO (kW) ISP ( $\mathrm{sec}$ ) Value (kg/day) PO Index

$\begin{array}{lllll}8500.00 & 100.00 & 15000.00 & 15.83359095 & 17 \\ 7500.00 & 100.00 & 16000.00 & 14.33637632 & 15 \\ 7500.00 & 100.00 & 20000.00 & 11.54509568 & 15 \\ 9000.00 & 100.00 & 22000.00 & 11.09327925 & 18 \\ 9000.00 & 100.00 & 24000.00 & 10.18881106 & 18\end{array}$



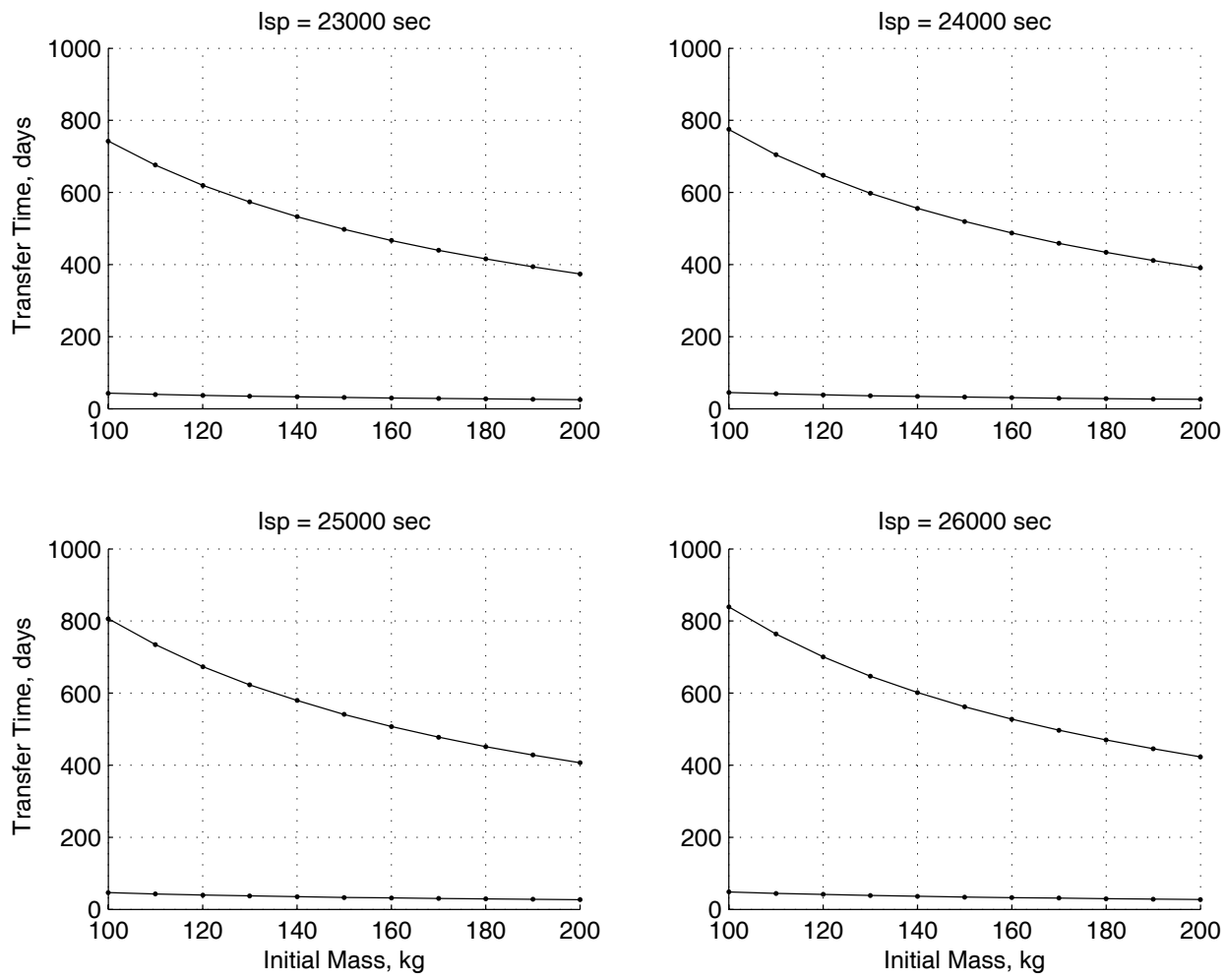

Figure D.11: Power vs. Transfer Time 

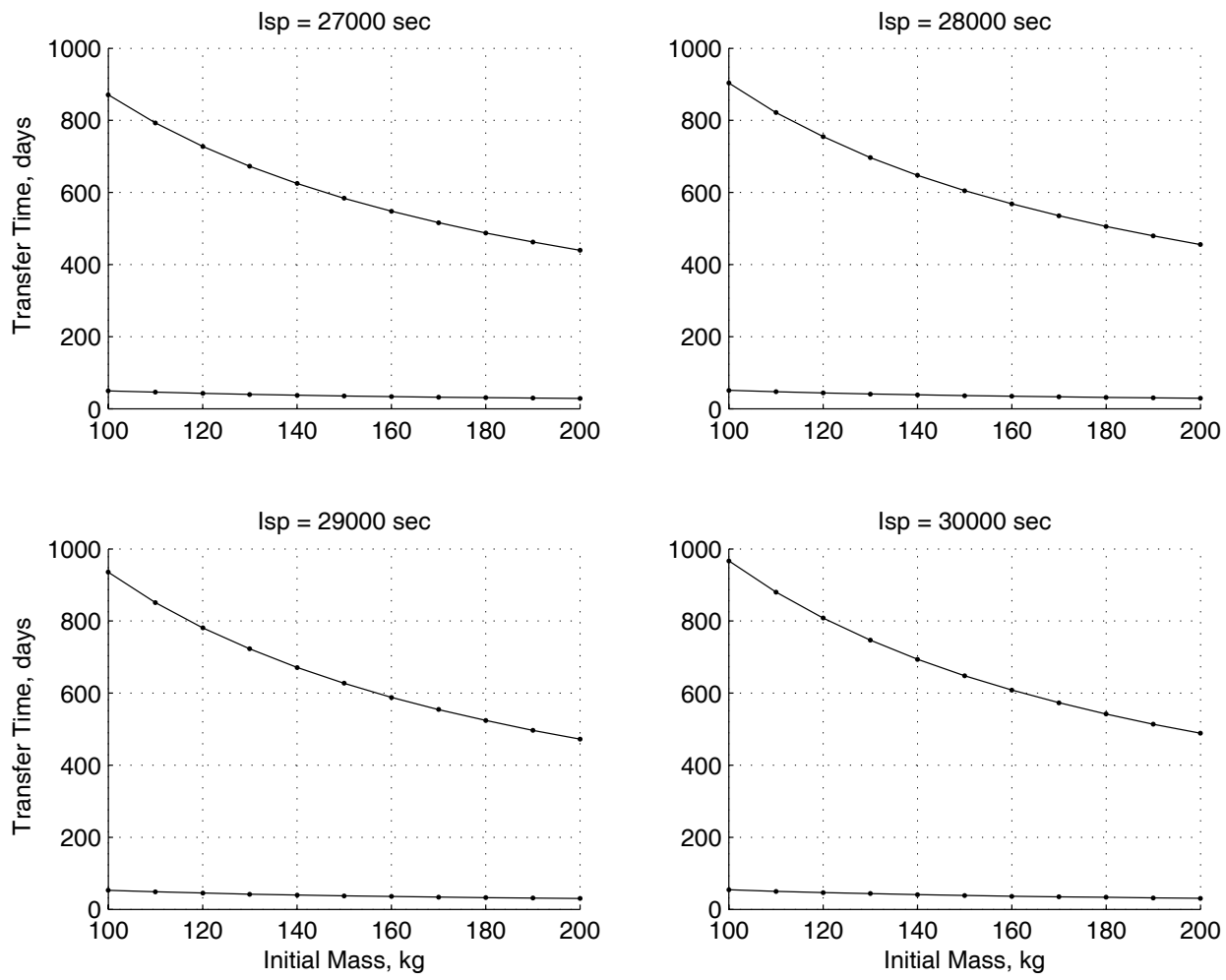

Figure D.12: Power vs. Transfer Time 


\begin{tabular}{|c|c|c|c|}
\hline 8500.00 & 100.00 & 25000.00 & 9.65175075 \\
\hline 8000.00 & 100.00 & 26000.00 & 9.13238262 \\
\hline 9000.00 & 100.00 & 27000.00 & 9.08027522 \\
\hline 9000.00 & 100.00 & 29000.00 & 8.47708513 \\
\hline 9000.00 & 100.00 & 30000.00 & 8.20209596 \\
\hline 8500.00 & 110.00 & 15000.00 & 17.08899094 \\
\hline 8500.00 & 110.00 & 16000.00 & 16.02705369 \\
\hline 9000.00 & 110.00 & 20000.00 & 13.11252370 \\
\hline 8500.00 & 110.00 & 21000.00 & 12.30034729 \\
\hline 8000.00 & 110.00 & 23000.00 & 11.05182707 \\
\hline 9000.00 & 110.00 & 24000.00 & 10.99179077 \\
\hline 9000.00 & 120.00 & 15000.00 & 18.60733602 \\
\hline 9000.00 & 120.00 & 16000.00 & 17.41860782 \\
\hline 8000.00 & 120.00 & 17000.00 & 15.84905871 \\
\hline 9000.00 & 120.00 & 29000.00 & 9.77834543 \\
\hline 9000.00 & 130.00 & 16000.00 & 18.57166874 \\
\hline 7500.00 & 130.00 & 21000.00 & 13.30061496 \\
\hline 9000.00 & 130.00 & 24000.00 & 12.47173371 \\
\hline 9000.00 & 140.00 & 17000.00 & 18.49773001 \\
\hline 9000.00 & 140.00 & 18000.00 & 17.46128566 \\
\hline 7500.00 & 140.00 & 22000.00 & 13.35785754 \\
\hline 9000.00 & 150.00 & 20000.00 & 16.52063180 \\
\hline 9000.00 & 150.00 & 27000.00 & 12.33910250 \\
\hline 8500.00 & 150.00 & 29000.00 & 11.25527876 \\
\hline 9000.00 & 160.00 & 19000.00 & 18.24467594 \\
\hline 9000.00 & 160.00 & 20000.00 & 17.33023016 \\
\hline 8500.00 & 160.00 & 21000.00 & 16.14638280 \\
\hline 9000.00 & 160.00 & 25000.00 & 13.90704128 \\
\hline 9000.00 & 200.00 & 27000.00 & 14.86558681 \\
\hline
\end{tabular}

The following are the tabulated results for the points of diminishing returns, where one exists for a combination of initial mass, power, and specific impulse, sorted by 
specific impulse.

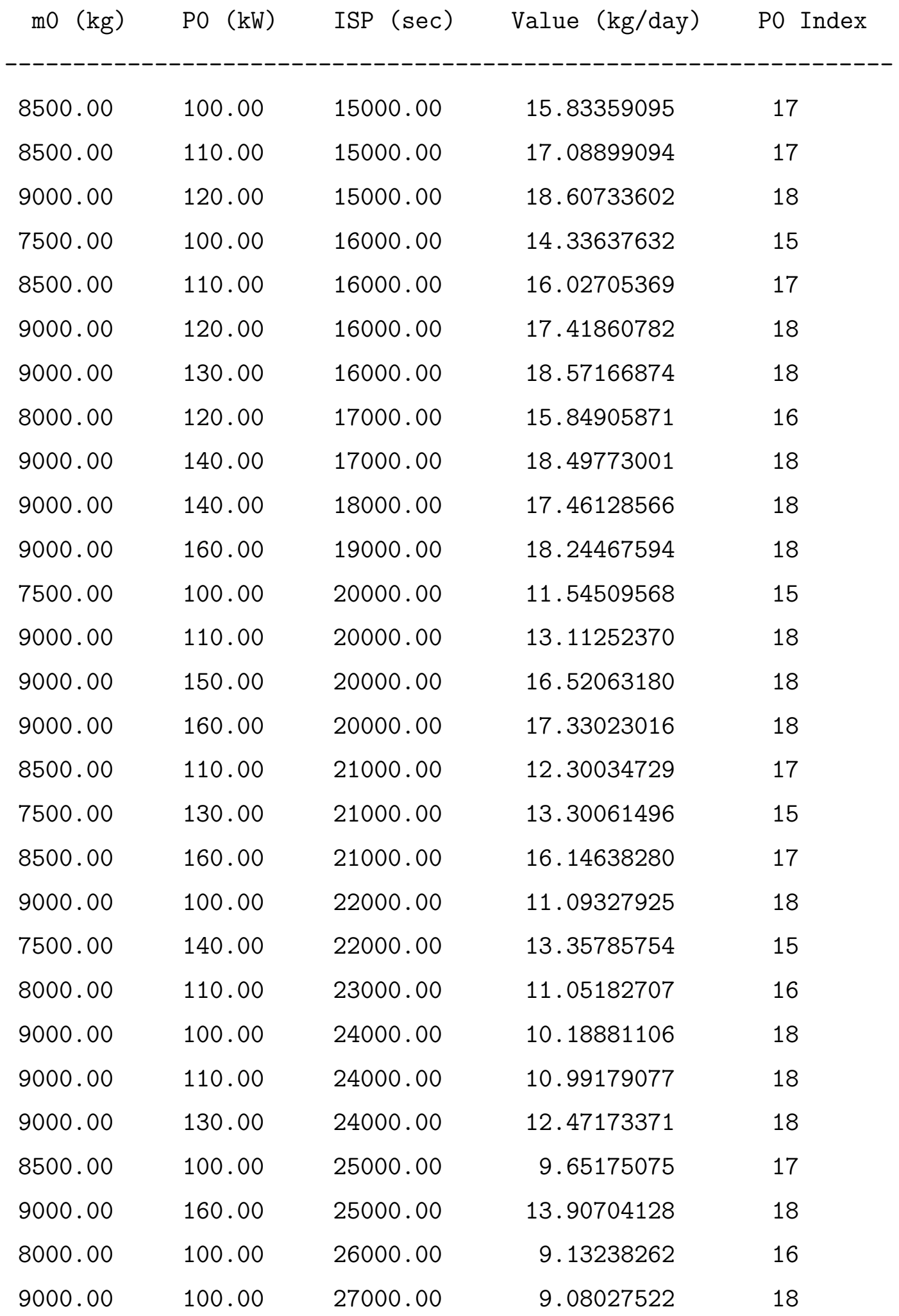




$\begin{array}{rrrrr}9000.00 & 150.00 & 27000.00 & 12.33910250 & 18 \\ 9000.00 & 200.00 & 27000.00 & 14.86558681 & 18 \\ 9000.00 & 100.00 & 29000.00 & 8.47708513 & 18 \\ 9000.00 & 120.00 & 29000.00 & 9.77834543 & 18 \\ 8500.00 & 150.00 & 29000.00 & 11.25527876 & 17 \\ 9000.00 & 100.00 & 30000.00 & 8.20209596 & 18\end{array}$

The following is the list of transfer times for the maximum optimum payload delivered per day.

$\begin{array}{cccc}\mathrm{m} 0(\mathrm{~kg}) & \mathrm{PO}(\mathrm{kW}) & \mathrm{ISP}(\mathrm{sec}) & \text { TOF (days) } \\ 9000.00 & 120.00 & 15000.00 & 362.9839 \\ 9000.00 & 130.00 & 16000.00 & 357.7031 \\ 9000.00 & 140.00 & 17000.00 & 353.1017 \\ 9000.00 & 140.00 & 18000.00 & 374.7898 \\ 9000.00 & 160.00 & 19000.00 & 345.6970 \\ 9000.00 & 160.00 & 20000.00 & 364.5350 \\ 8500.00 & 160.00 & 21000.00 & 361.5730 \\ 7500.00 & 140.00 & 22000.00 & 383.0115 \\ 8000.00 & 110.00 & 23000.00 & 542.4210 \\ 9000.00 & 130.00 & 24000.00 & 539.2745 \\ 9000.00 & 160.00 & 25000.00 & 457.1446 \\ 8000.00 & 100.00 & 26000.00 & 672.6456 \\ 9000.00 & 200.00 & 27000.00 & 395.6584 \\ 8500.00 & 150.00 & 29000.00 & 534.1492 \\ 9000.00 & 100.00 & 30000.00 & 871.1938\end{array}$




\section{Bibliography}

[1] Report of the President's Commission on Implementation of United States Space Exploration Policy. June 2004.

[2] James G. Watzin, Joseph Burt, and Craig Tooley. The Robotic Lunar Exploration Program (RLEP) - and introduction to the goals, approach, and architecture. Number AIAA 2005-2548, February 2005. 1st Space Exploration Conference: Continuing the Voyage of Discovery.

[3] Lunar architecture focused trade study final report. Technical Report ESMDRQ-0005, National Aeronautics and Space Administration, Headquarters, Washington DC 20546-0001, Oct 2005. Baseline.

[4] Mark Beckman and Dave Folta. Mission design of the first robotic lunar exploration program mission: The lunar reconnaissance orbiter. Aug 2005. AAS/AIAA Astrodynamics Specialist Conference.

[5] Michael Braukus and J.D. Harrington. NASA selects team to build lunar lander. NASA Website, Sept 2005. Press Release 05-289.

[6] David Y. Oh, Thomas Randolph, Manuel Martinez-Sanchez, and Scott Kimbrel. End to end optimization of two dimensional chemical-electric orbit raising missions. AIAA, July 2002. 38th AIAA/ASME/SAE/ASEE Joint Propulsion Conference and Exhibit.

[7] David Y. Oh and Glenn Santiago. Analytic optimization of mixed chemicalelectric orbit raising missions. Electric Rocket Propulsion Society, October 2001. 27th International Electric Propulsion Conference. 
[8] Muriel Noca and John R. Brophy. Over powering solar system exploration. July 1997. AIAA-1997-2914.

[9] Ronald Spores et al. A solar electric propulsion cargo vehicle to support NASA lunar exploration program. (IEPC-2005-320), 2005.

[10] The Boeing Company, CA. Delta II: Payload Planners Guide, Oct 2000. MDC $00 \mathrm{H} 0016$.

[11] Philip G. Hill and Carl R. Peterson. Mechanics and Thermodynamics of Propulsion. Addison-Wesley Publishing Company, New York, second edition, 1992.

[12] Robert G. Jahn. Physics of Electric Propulsion. McGraw-Hill Book Company, New York, 1968.

[13] David Manzella, Robert Jankovsky, and Richard Hofer. Laboratory model 50 kw hall thruster. July 2002. 38th AIAA/ASME/SAE/ASEE Joint Propulsion Conference and Exhibit.

[14] Frank Gulczinski III and Ronald A. Spores. Analysis of hall-effect thrusters and ion engines for orbit transfer missions. July 1996. AIAA 96-2973.

[15] Christian Carpenter. Mission analysis for spacecraft using direct drive technology. Personal communication, 2005.

[16] W. Andrew Hoskins et al. Direct drive hall thruster system development. July 2003. 39th AIAA/ASME/SAE/ASEE Joint Propulsion Conference and Exhibit.

[17] James R. Wertz and Wiley J. Larson, editors. Space Mission Analysis and Design. Space Technology Library. Kluwer Academic Publishers and Microcosm Press, El Segundo, California, third edition, 1999.

[18] The Boeing Company, Huntington Beach, CA. Delta IV: Payload Planners Guide, Oct 2000. MDC 00H0043.

[19] International Launch Services, CA. Atlas Launch System: Mission Planner's Guide, Dec 1999. CLSB-9911-0586. 
[20] R. Bertrand, G. Klingelhofer, R. Rieder, M. van Winnendael, and M. Zelikman. European tracked micro-rovers for planetary surface exploration. July 2000. Concepts and Approaches for Mars Exploration.

[21] James J. Zakrajsek, David B. McKissock, Jeffrey M. Woytach, and et al. Exploration rover concepts and development challenges. Feb 2005. First Space Exploration Conference: Continuing the Voyage of Discovery.

[22] M. Rai-Rohani. On structural design of a mobile lunar habitat with multi-layered environmental shielding. NASA contractor report, NASA Marshall Space Flight Center, 2005. NASA/CR 2005-213845.

[23] Christian B Carpenter. Electrically propelled cargo spacecraft for sustained lunar supply operations. July 2006. AIAA 2006-4435.

[24] N. Sitnikova et al. Hall effect thruster interactions data from the russian expressa2 and express-a3 satellites: Acquire express-a2 spt-100 based propulsion subsystem and other subsystem flight operation tm-data for the period of march 12 , 2000 to and including june 15, 2000, task 29. NASA contractor report, NASA Marshall Space Flight Center, 2003. NASA/CR2003-212005.

[25] Peter Rathsman, Joakim Kugelberg, Per Bodin, Giuseppe D. Racca, Bernard Foing, and Luca Stagnaro. SMART-1: Development and lessons learnt. Acta Astronautica, 57:455-468, 2005. 